\title{
L'insertion professionnelle des diplômés des cycles supérieurs
}

Sous la direction de Wenceslas Mamboundou et Salim Laaroussi
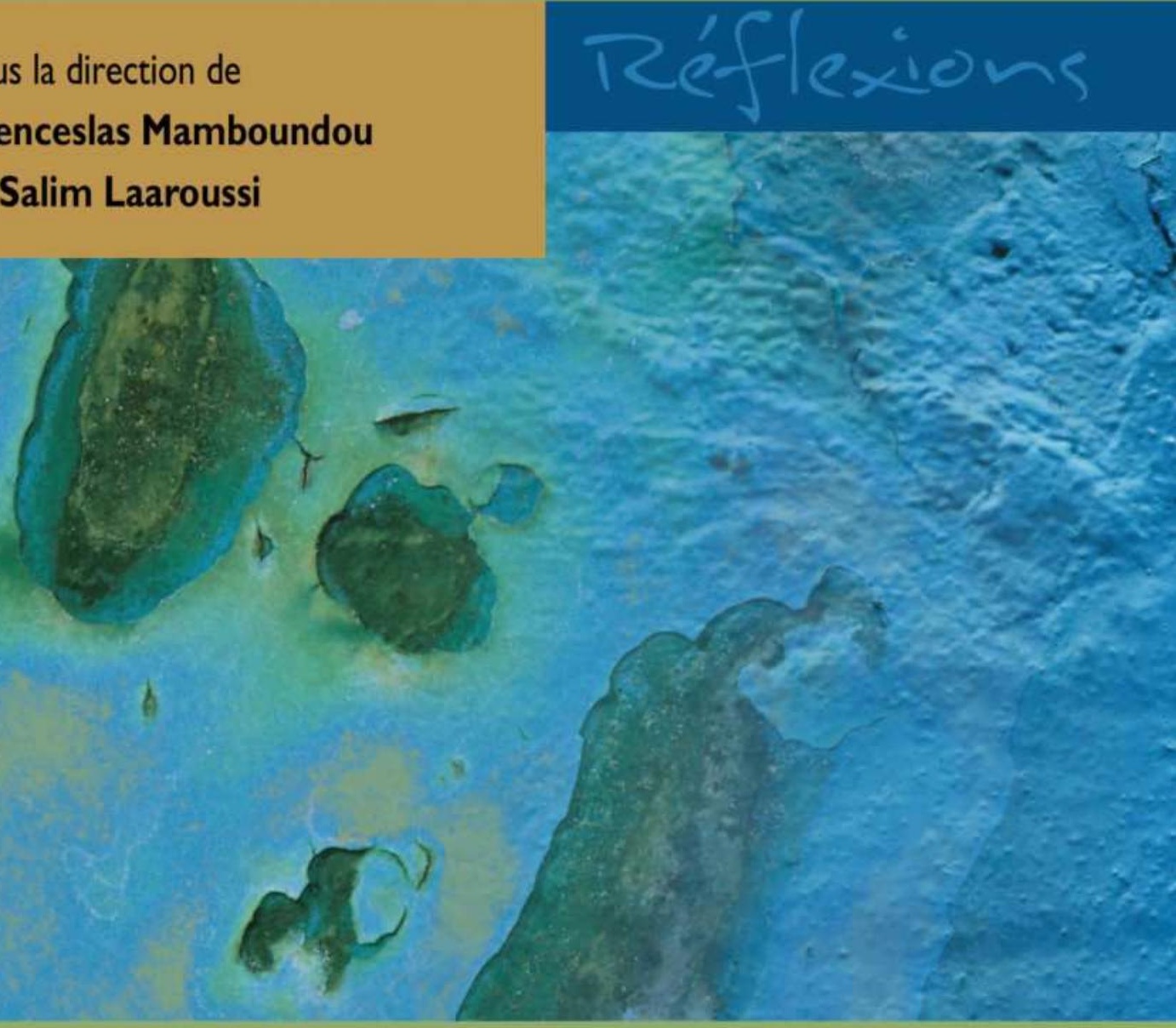

Presses

de I'Université du Québec 

L'insertion professionnelle des diplômés des cycles supérieurs

Réflexions 


\section{PRESSES DE L'UNIVERSITÉ DU QUÉBEC}

Le Delta I, 2875, boulevard Laurier, bureau 450

Sainte-Foy (Québec) G1V 2M2

Téléphone: (418) 657-4399 • Télécopieur: (418) 657-2096

Courriel: puq@puq.ca・Internet : www.puq.ca

Distribution :

\section{CANADA et autres pays}

Distribution DE LIVRES UNIVERS S.E.N.C.

845, rue Marie-Victorin, Saint-Nicolas (Québec) G7A 3S8

Téléphone : (418) 831-7474/1-800-859-7474 • Télécopieur: (418) 831-4021

\section{FRANCE}

Distribution du Nouveau Monde

30, rue Gay-Lussac, 75005 Paris, France

Téléphone: 33143544902

Télécopieur: 33143543915

\section{SUISSE}

\section{SERVIDIS SA}

5, rue des Chaudronniers, CH-1211 Genève 3, Suisse Téléphone: 0229609525

Télécopieur: 0227763527
DANGER

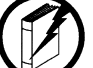

PHOTOCOPILLA

TUE LE LIVRE
La Loi sur le droit d'auteur interdit la reproduction des œuvres sans autorisation des titulaires de droits. Or, la photocopie non autorisée - le «photocopillage »s'est généralisée, provoquant une baisse des ventes de livres et compromettant la rédaction et la production de nouveaux ouvrages par des professionnels. L'objet du logo apparaissant ci-contre est d'alerter le lecteur sur la menace que représente pour l'avenir de l'écrit le développement massif du «photocopillage». 


\section{L'insertion professionnelle des diplômés des cycles supérieurs}

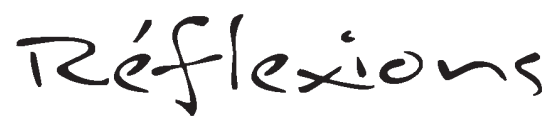

Sous la direction de

Wenceslas Mamboundou

et Salim Laaroussi 
Vedette principale au titre:

L’insertion professionnelle des diplômés des cycles supérieurs: réflexions

Textes présentés lors d'un colloque organisé par le Conseil national des cycles supérieurs dans le cadre du Congrès de l'Acfas et tenu le 11 mai 2004.

Comprend des réf. bibliogr.

ISBN 2-7605-1394-7

1. Diplômés d'université - Travail - Québec (Province) - Congrès.

2. Insertion professionnelle - Québec (Province) - Congrès.

3. Qualifications professionnelles - Québec (Province) - Congrès.

I. Mamboundou, Wenceslas, 1965- ․ II. Laaroussi, Salim. III. Fédération étudiante universitaire du Québec. Conseil national des cycles supérieurs.

IV. Congrès de l'Acfas (72 : 2004 : Université du Québec à Montréal).

HD6278.C32Q867 $2005 \quad$ 331.11'4235'09714 C2005-941795-1

Nous reconnaissons l'aide financière du gouvernement du Canada par l'entremise du Programme d'aide au développement de l'industrie de l'édition (PADIÉ) pour nos activités d'édition.

La publication de cet ouvrage a été rendue possible avec l'aide financière de la Société de développement des entreprises culturelles (SODEC).

Mise en pages : INFo IOOO MOTS INC.

Couverture : RICHARD HODGSON

\section{PUQ 2005987654321}

Tous droits de reproduction, de traduction et d'adaptation réservés (C) 2005 Presses de 1’Université du Québec

Dépôt légal $-4^{e}$ trimestre 2005

Bibliothèque nationale du Québec / Bibliothèque nationale du Canada

Imprimé au Canada 


\section{AVANT-PROPOS}

Le Conseil national des cycles supérieurs dew la Fédération étudiante universitaire du Québec (CNCS-FEUQ) est un lieu de débats, de discussions, de réflexions, de prises de positions et d'actions visant à représenter et à défendre les étudiants des cycles supérieurs. Il regroupe 13 associations membres et représente près de 30000 étudiants de $2^{\mathrm{e}}$ et $3^{\mathrm{e}}$ cycles des universités du Québec.

Dans le cadre d'une problématique qui préoccupe particulièrement ses membres, cet ouvrage vous présente les réflexion $s$ d'un ensemble d'acteurs et d'organismes qui s'intéressent à la recherche et à la formation des chercheurs. Nous osons espérer qu'il contribuera à une meilleure compréhension du phénomène de l'insertion des diplômés des cycles supérieurs et à un ajustement des politiques actuellement en vigueur, de manière à favoriser la pleine contribution des ciplômés dans la production des savoirs.

En plus, le CNCS souhaiterait remercier, pour leur contribution financière et leur appui inestimable, les partenaires qui nous ont permis l'organisation de ce colloque de mai 2004 et la publication de cet ouvrage: 
- Ministère du Développement économique, de l'Innovation et de l'Exportation;

- Ministère de l'Éducation, du Loisir et du Sport;

- Fonds québécois de recherche sur la société et la culture;

- Fonds québécois de recherche sur la nature et les technologies;

- Conseil de recherche en sciences naturelles et en génie du Canada;

- Conseil de recherche en sciences humaines du Canada.

Enfin, nos remerciements vont également à l'endroit de Guillaume Bélanger, permanent à la recherche de la Fédération étudiante universitaire du Québec, pour sa contribution à la première mise en forme de cet ouvrage ainsi que pour ses diverses suggestions.

Philippe-Olivier GIroux Président du CNCS 


\section{TABLE DES MATIÈRES}

Introduction. ..................................... 1

Wenceslas Mamboundou, Ph.D. et Salim Laaroussi, Ing.

Partie 1

Savoirs et expériences partagés :

une approche plurielle de la question

de l'insertion socioprofessionnelle des diplômés

des cycles supérieurs

Chapitre 1 Le marché du travail pour les diplômés des cycles supérieurs

Hélène P.Tremblay

Le flux de nouveaux diplômés ne forme qu'un des piliers

de l'offre de main-d'œuvre hautement qualifiée. .......... 7

Des comportements qui s'adaptent à la conjoncture ........ 8

Absence de pénurie généralisée de THQ à l'horizon . . . . . . . . . 9 
Chez les diplômés des cycles supérieurs,

des disparités importantes entre domaines disciplinaires....... 10

Les leçons à tirer ................................. 12

Chapitre 2 Qu'advient-il des diplômés

des cycles supérieurs?

MM. Pierre Michel et François Girard

Les objectifs des enquêtes

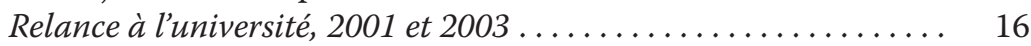

La méthodologie et le recensement................... 16

Quelques caractéristiques des personnes diplômées ......... 16

L'emploi chez les personnes diplômées................... 19

Conclusion ................................ 25

Annexe - Définitions ....................... 26

Chapitre 3 Le rôle stratégique des organismes

subventionnaires dans le développement

de la recherche universitaire et de la relève

scientifique au Québec

Louise Dandurand

Les grands enjeux et principales pressions contextuelles ...... 30

La place de la relève dans la mission des Fonds............ 33

En conclusion............................... 40

Chapitre 4 Renforcer l'acquisition des compétences

et la gestion des carrières $\ldots \ldots \ldots \ldots \ldots \ldots \ldots, 43$

Sylvie Dillard

Les trois grands défis du Québec .................... 44

Des pistes d'action pour l'avenir.................... 48

Chapitre 5 Le renouvellement et le développement

du corps professoral universitaire:

l'identification des besoins des universités

québécoises

Jean-Pierre Proulx

La méthodologie ... 
La collecte de données ........................... 53

Les prévisions $2003-2008 \ldots \ldots \ldots \ldots \ldots \ldots \ldots \ldots \ldots \ldots$. 54

Les prévisions selon les caractéristiques des universités $\ldots \ldots \ldots \quad 56$

Chapitre 6 Formation en milieu pratique et insertion professionnelle des jeunes chercheurs .

Brigitte Gemme et Yves Gingras

L'enquête...................................... 60

Résultats $\ldots \ldots \ldots \ldots \ldots \ldots \ldots \ldots \ldots \ldots \ldots \ldots \ldots \ldots, 62$

Conclusion ................................ 68

Bibliographie ............................. 71

Chapitre 7 Exode des cerveaux:

phénomène imaginaire?

Stéphanie Andrew et Daniel Parent

Description de l'approche ......................... 74

Résultats ............................... 77

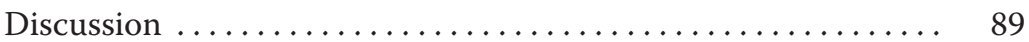

Bibliographie .............................. 91

Partie 2

\section{L'insertion socioprofessionnelle}

Le point de vue du Conseil national

des cycles supérieurs.

Chapitre 8 L'insertion, un concept en redéfinition

Mouvance et instabilité du marché de l'emploi:

miser sur l'employabilité de l'individu.................. 96

Représentations et attentes des diplômés face à l'insertion...... 99

Comprendre le processus d'insertion ................. 102

Les stratégies d'insertion ........................... 106 


\section{Chapitre 9 Favoriser l'insertion optimale} des diplômés des cycles supérieurs

Comment intervenir sur l'insertion $? \ldots \ldots \ldots \ldots \ldots \ldots \ldots . . \ldots 111$

Les facteurs structuraux influençant l'insertion ............ 113

Comment concevoir l'insertion optimale ?.............. 115

\section{Chapitre 10 L'élaboration d'un projet professionnel} et l'orientation

Le manque chronique de ressources consacrées

à l'orientation ............................... 118

L'orientation, un processus continu................... 119

Les moyens appropriés pour assurer un projet

d'insertion optimal ............................. 121

\section{Chapitre 11 La formation et le développement} des compétences.

Une adaptation de la formation à la recherche dans les universités

Le développement des compétences dans un objectif stratégique de planification professionnelle

Une meilleure connaissance des compétences recherchées ..... 130

Une offre accrue de formations courtes pouvant préparer

le diplômé à s'insérer plus efficacement .................. 132

La formation et les compétences à développer

lors de linsertion............................. 134

\section{Chapitre 12 La capacité d'innover et la mise} en valeur des diplômés

Une meilleure connaissance des compétences

des diplômés de cycles supérieurs . . . . . . . . . . . . . . . . . 142

L'insertion de diplômés de cycles supérieurs pour accroître la capacité d'innover.................... 143

Le soutien des efforts des diplômés de cycles supérieurs pour favoriser l'innovation 


\section{LISTE DES FIGURES}

Figure 1.1 Synthèse des principaux constats relatifs

à l'adéquation de l'offre et de la demande

Figure 3.1 Pressions contextuelles sur le système de recherche et d'innovation $\ldots \ldots \ldots \ldots \ldots \ldots \ldots \ldots . \quad 31$

Figure 3.2 Budget des subventions au FQRNT et au FQRSC ........ 37

Figure 7.1a Canadiens ayant un doctorat et travaillant aux É.-U. Personnes œuvrant dans tous les secteurs ........... 79

Figure 7.1b Canadiens-français ayant un doctorat et travaillant aux É.-U. - Personnes œuvrant dans tous les secteurs ..... . 79

Figure 7.2a Canadiens ayant un doctorat et travaillant aux É.-U. Secteur de l'éducation....................... 80

Figure 7.2b Canadiens-français ayant un doctorat et travaillant aux É.-U. - Secteur de l'éducation ................. 80

Figure 7.3a Immigrants ayant un doctorat et travaillant au Canada Personnes œuvrant dans tous les secteurs ............ 81

Figure 7.3b Immigrants ayant un doctorat et travaillant au Canada Secteur de l'éducation....................... 81

Figure 7.4a Distribution de lâge des émigrants canadiens ........... 84 
Figure 7.4b Distribution de l'âge des émigrants récents........... 84

Figure 7.5a Âge des Canadiens au Canada (hommes) ............. 85

Figure 7.5b Âge des émigrants canadiens aux É.-U. (hommes) ........ 85

Figure 7.5c Âge des immigrants au Canada (hommes) ............ 86 


\section{LISTE DES TABLEAUX}

Tableau 1.1 Situation en 2001 ou 2003 des diplômés de maîtrise promus en 1999 ou $2001 \ldots \ldots \ldots \ldots \ldots \ldots \ldots \ldots \ldots \ldots$

Tableau 1.2 Situation en janvier 2001 des titulaires d'un doctorat de la promotion 1999 selon le domaine de formation. . . . . .

Tableau 2.1 Situation en 2003 des titulaires d'une maîtrise de 2001

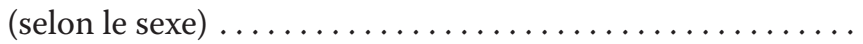

Tableau 2.2 Situation en 2003 des titulaires d'une maîtrise de 2001 (selon le domaine d'études) .

Tableau 2.3 Situation en 2001 des titulaires d'un doctorat de 1999 (selon le sexe)

Tableau 2.4 Situation en 2001 des titulaires d'un doctorat de 1999

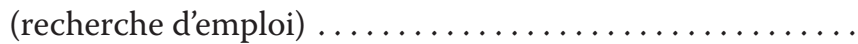

Tableau 2.5 Situation en 2003 des titulaires d'une maîtrise de 2001 (recherche d'emploi) .........................

Tableau 2.6 Situation en 2003 des titulaires d'une maîtrise de 2001 (caractéristiques de l'emploi).

Tableau 2.7 Situation en 2001 des titulaires d'un doctorat de 1999 (caractéristiques de l'emploi) 
Tableau 2.8 Situation en 2003 des titulaires d'une maîtrise de 2001 (statut d'emploi) .............................

Tableau 2.9 Situation en 2001 des titulaires d'un doctorat de 1999

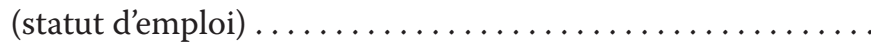

Tableau 2.10 Situation en 2003 des titulaires d'une maitrise de 2001 (taille des entreprises) .........................

Tableau 2.11 Situation en 2001 des titulaires d'un doctorat de 1999 (taille des entreprises) ........................

Tableau 2.12 Situation en 2001 des titulaires d'une maîtrise de 2001 (chômage) .................................

Tableau 2.13 Situation en 2001 des titulaires d'un doctorat de 1999

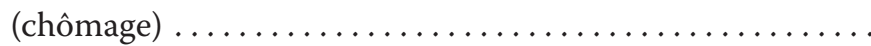

Tableau 3.1 Étudiants de $2^{\mathrm{e}}$ et $3^{\mathrm{e}}$ cycle..................... 34

Tableau 3.2 Stagiaires postdoctoraux $\ldots \ldots \ldots \ldots \ldots \ldots \ldots \ldots \ldots \ldots$

Tableau 3.3 Bourses d'excellence (2002-2003) reçues par des étudiants et postdoctorants en provenance des Fonds de recherche du Québec ..................

Tableau 3.4 Programmes de subventions ciblés pour le soutien au démarrage de carrière en recherche (2003-2004) .......

Tableau 3.5 Parts des subventions de recherche (2001-2002) reçues par des étudiants et postdoctorants en provenance des Fonds de recherche du Québec ..................

Tableau 4.1 Recherche universitaire et R-D-I (Recherche Développement - Innovation) en entreprise: une nature et des objectifs différents ................

Tableau 5.1 Variation de l'effort de recrutement prévu selon les universités........................... 54

Tableau 5.2 Les prévisions selon les caractéristiques des universités .... 56

Tableau 6.1 Distribution des répondants par catégorie ............ 62

Tableau 6.2 Projets professionnels des étudiants «L'emploi que vous aimeriez le plus occuper dans l'avenir serait-il plutôt...»..................

Tableau 6.3 Intention de faire de la recherche «Quelle place souhaitez-vous que la recherche occupe dans votre carrière?» 
Tableau 6.4 Sentiment de préparation à l'égard du marché du travail - Étudiants «Comment vous sentez-vous par rapport à votre entrée sur le marché du travail ?» .....

Tableau 6.5 Occupation des répondants «Lequel des énoncés suivants s'applique le mieux à votre situation?» ........

Tableau 6.6 Milieu de travail «À laquelle des catégories suivantes appartient votre employeur actuel ?»........ 66

Tableau 6.7 Temps requis pour l'insertion professionnelle «Combien de temps s'est écoulé entre le moment où vous avez obtenu votre diplôme et le moment où vous avez trouvé un emploi ?»...............

Tableau 6.8 Sentiment de préparation à l'égard du marché du travail - Diplômés «Selon vous, comment votre programme vous a-t-il préparé, jusqu'à présent, pour le marché du travail $»^{\prime} \ldots \ldots \ldots \ldots \ldots \ldots \ldots \ldots$

Tableau 6.9 Nombre et catégories d'acteurs soutenant l'étudiant dans son mémoire (sa thèse) «Lesquelles des personnes suivantes vous ont fourni de l'aide ou du soutien dans la réalisation de votre projet de recherche ${ }^{*} ? » . \ldots \ldots$

Tableau 7.1 Salaire des Canadiens de naissance ayant un doctorat et travaillant aux États-Unis comparé au salaire des Canadiens titulaires de doctorat au Canada. . . . . . . . . . .

Tableau 7.2 Salaire des Canadiens de naissance ayant un doctorat et travaillant aux États-Unis comparé au salaire des Américains détenteurs de doctorat aux É.-U. . . . . . . . .

Tableau 7.3 Salaire des Canadiens de naissance ayant un doctorat et travaillant au Canada comparé au salaire des immigrants détenteurs de doctorat au Canada........

Tableau 12.1 Entraves à l'innovation . . . . . . . . . . . . . . . . . . . . . . 151 



\section{INTRODUCTION}

Wenceslas Mamboundou, Ph. D.

et Salim Laaroussi, Ing.

Depuis plusieurs années, les discours tenus par certains étudiants et diplômés de cycles supérieurs et par certains employeurs n'ont pas manqué de soulever des questions sur l'insertion professionnelle. Les diplômés de cycles supérieurs prétendent que leur diplôme ne leur assure plus un travail répondant à leurs attentes. Pourtant, les universités et les gouvernements promeuvent plus que jamais les études de $2^{\mathrm{e}}$ et $3^{\mathrm{e}}$ cycle et la formation à la recherche.

Pour le monde universitaire, aussi bien que pour l'ensemble des secteurs de l'économie, les études aux $2^{\mathrm{e}}$ et $3^{\mathrm{e}}$ cycles constituent un gage de succès du développement de notre société. Par exemple, le Council on Competitiveness aux États-Unis considère que le déclin des ressources nationales consacrées à la recherche « est un facteur de vulnérabilité qui menace à long terme la capacité d'innover [d'une société] ${ }^{1} »$.

La recherche universitaire apparaît ainsi comme un puissant moteur du développement économique. Par exemple, au Canada, l'impact direct de la recherche universitaire s'estimait à plus de cinq milliards de dollars

1. Cité par l'Association canadienne pour les études supérieures dans «Former des têtes bien faites pour l'économie du savoir : baliser le chemin vers le succès », exposé d'intention préparé à l'intention du Comité de direction, octobre 2001. 
du PIB en 1994-1995. Elle a également une influence dynamique sur la productivité de l'économie. "L'impact dynamique de la recherche universitaire s'élève à approximativement 15,5 milliards de dollars chaque année, ce qui équivaut à environ 150000 à 200000 emplois $^{2}$.»

Il faut éviter de nous asseoir sur nos lauriers. Les pressions exercées par les changements économiques, technologiques et scientifiques, le vieillissement de la main-d'œuvre et un marché des compétences qui se mondialise et qui devient de plus en plus compétitif sont des facteurs clés qui, ensemble, mettront bientôt à très rude épreuve notre système de perfectionnement des compétences. [...] Nos systèmes d'enseignement et de formation montrent des signes évidents de fatigue. [...] Nos collèges et universités ont été sérieusement affaiblis par les compressions budgétaires de la dernière décennie ${ }^{3}$.

Depuis 1992, les universités canadiennes éprouvent des difficultés à remplacer leurs professeurs retraités compte tenu des restrictions budgétaires. L'Association des universités et collèges du Canada (AUCC) estime qu'elles devront recruter, d'ici 2010, en moyenne entre 2500 et 3000 professeurs par an afin de combler les nombreux postes vacants. $\mathrm{Au}$ Québec, les estimations varient entre 8000 et 10000 nouveaux professeurs au cours de la prochaine décennie. Avec seulement $40 \%$ des détenteurs d'un doctorat qui font carrière dans le milieu universitaire canadien, notre système universitaire se fragilisera si nous n'apportons pas de réponses appropriées.

A priori, un diplômé des cycles supérieurs ne devrait pas avoir de difficultés à se trouver un emploi, en raison de son niveau d'instruction. Les statistiques officielles appuient d'ailleurs cette hypothèse puisque le taux de chômage est généralement plus bas pour les diplômés d'études postsecondaires que pour la moyenne des Québécois et il est encore plus bas pour les diplômés des cycles supérieurs. Malgré des taux de placement élevés, il se véhicule une fausse représentation de l'insertion professionnelle des diplômés des cycles supérieurs. Les enjeux de l'employabilité des diplômés de cycles supérieurs ont rarement été explicités, entre autres parce que les statistiques d'emploi les concernant ont toujours été positives. Comme ils sont les plus qualifiés, on présume que

2. Conseil national des cycles supérieurs (CNCS), Pour innover, investir dans la relève, 2002, p. 15.

3. Groupe d'experts du Conseil consultatif des sciences et de la technologie (CCST) sur les compétences: Viser plus haut: compétences et esprit d'entreprise dans l'économie du savoir, $<$ http/acst-ccst.gc.ca/acst/skills/finalrepdocs/ccst-s.pdf $>$. 
leur insertion en emploi va de soi. Cependant, on néglige le fait que notre économie n'a pas été préparée à les accueillir, phénomène qui accentue le risque de les voir sous-utilisés ou mal utilisés.

Pourquoi les diplômés considèrent que les emplois se font rares dans leur domaine? Comment se fait-il qu'un diplôme de $2^{\mathrm{e}}$ et $3^{\mathrm{e}}$ cycle puisse devenir un frein à l'employabilité? Quelles sont les perceptions des employeurs à l'égard des diplômés des cycles supérieurs? Ces derniers, répondent-ils aux exigences des employeurs? Les perceptions des employeurs et celles des diplômés proviendraient-elles d'une mauvaise promotion des études conduisant à l'obtention d'une maitrise ou d'un doctorat? Les besoins de notre société sont-ils comblés?

Lors d'un colloque qui a été organisé en mai 2004 par le Conseil national des cycles supérieurs (CNCS) dans le cadre du congrès de l'Association canadienne-française pour l'avancement des sciences (ACFAS), plusieurs experts se sont prononcés et ont tenté de répondre à ces questions. Le but de cet exercice était de nourrir la réflexion sur cette problématique. Cet ouvrage, qui résulte de ce colloque, se divise en deux parties. La première regroupe les présentations des divers conférenciers. Quant à la deuxième partie, elle résume une recherche menée par le CNCS sur le sujet.

Le premier chapitre reprend pour l'essentiel un avis publié par le Conseil de la science et de la technologie sur la main-d'œuvre hautement qualifiée au Québec. Il traite de la dynamique globale qui structure le marché du travail pour les détenteurs d'un diplôme d'études postsecondaires, ce qui inclut évidemment la question de leur insertion professionnelle.

Le deuxième chapitre présente les résultats d'une enquête réalisée par le ministère de l'Éducation sur «La relance à l'université». Cette enquête est menée aux deux ans auprès des titulaires d'un baccalauréat, d'une maitrise et d'un doctorat.

Le troisième chapitre analyse le rôle stratégique joué par les trois Fonds subventionnaires du Québec dans le système de recherche et d'innovation en matière de soutien à la relève, dans ses dimensions de vocation, de formation et de carrière. Un survol des grands enjeux et de quelques pressions contextuelles qui influencent les besoins de relève scientifique sera également effectué. 
Le quatrième chapitre insiste sur la question des compétences et de la formation pour comprendre la problématique de l'insertion des diplômés des cycles supérieurs. Car, pour prendre leur place dans cette société du savoir et de l'innovation, les diplômés doivent pouvoir répondre aux exigences du marché du travail.

Le cinquième chapitre analyse l'ancien programme de Bourses de recherche en milieu de pratique du Fonds québécois de recherche sur la nature et les technologies. Â partir d'une enquête réalisée auprès des bénéficiaires de ce programme, il a été possible d'estimer la qualité de leur formation à la recherche et les impacts du programme sur leur insertion professionnelle.

Le sixième chapitre s'attarde au dernier rapport annuel sur le renouvellement du corps professoral universitaire québécois publié par le Conseil supérieur de l'éducation.

Le septième chapitre traite de la problématique de l'insertion sous l'angle de l'«exode des cerveaux». À l'aide des données des recensements américains et canadiens au cours de la période allant de 1970 à 2000, l'auteur compare les flux d'immigration au Canada et d'émigration du Canada vers les États-Unis, particulièrement pour les titulaires de Ph. D.

En deuxième partie, cet ouvrage suggère des solutions en se basant sur une étude menée par le CNCS. Ainsi, le chapitre 8 pose un regard critique sur la définition du concept d'insertion. Le chapitre 9 relève la nécessité de favoriser l'insertion optimale des diplômés des cycles supérieurs. Le chapitre 10 aborde la question sous l'angle de l'élaboration d'un projet professionnel, c'est-à-dire celui du développement des compétences. Le chapitre 11 porte sur la formation à la recherche universitaire en mettant l'accent sur l'urgence d'adapter la formation dans le cadre des compétences. Enfin, le douzième et dernier chapitre souligne la capacité d'innover et la mise en valeur des diplômés des cycles supérieurs dans le contexte d'une économie du savoir. 


\section{Première partie}

\section{SAVOIRS ET EXPÉRIENCES PARTAGÉS}

Une approche plurielle de la question de l'insertion socioprofessionnelle des diplômés des cycles supérieurs 



\section{Chapitre}

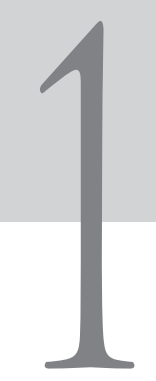

\section{LE MARCHÉ DU TRAVAIL POUR LES DIPLÔMÉS DES CYCLES SUPÉRIEURS}

\section{Hélène P. Tremblay}

Présidente, Conseil de la science

et de la technologie

Le Conseil de la science et de la technologie vient de faire paraître un avis sur l'état du marché pour les travailleurs hautement qualifiés (THQ) ${ }^{1}$. Bien que les THQ comprennent l'ensemble des diplômés collégiaux (filière technique) et universitaires, certains passages de l'avis traitent spécifiquement des finissants des cycles supérieurs ou avancent des constats qui s'appliquent parfaitement à ces derniers. Le présent texte reviendra brièvement sur les résultats les plus pertinents relativement à la question de l'insertion professionnelle.

\section{LE FLUX DE NOUVEAUX DIPLÔMÉS NE FORME QU'UN DES PILIERS DE L'OFFRE DE MAIN-D'CEUVRE HAUTEMENT QUALIFIÉE}

Ces années-ci, les nouveaux diplômés universitaires constituent environ $3 \%$ du bassin de THQ. Leur comportement a donc un effet significatif sur le marché. Mais d'autres piliers sont également à l'œuvre du côté de

1. Conseil de la science et de la technologie, L'avenir de la main-d'ouvre hautement qualifiée. Une question d'ajustements, 2004. Le document intégral est disponible dans le site <www.cst. gouv.qc.ca>. 
l'offre. Par exemple, au moins 15000 immigrants hautement qualifiés sont entrés au Québec en 2002, soit l'équivalent de $30 \%$ des diplômés détenant un niveau de scolarité comparable. Les employeurs peuvent également recourir à la formation continue pour rehausser les compétences de leurs employés et, ce faisant, leur permettre de combler des postes exigeant des qualifications plus élevées; cette stratégie est en revanche peu prisée au Québec, si l'on se fie à la faible participation des travailleurs québécois à des activités de formation en emploi. Enfin, l'entrée ou le retour des diplômés inactifs (à la retraite, à la maison, aux études...) peut aussi varier en fonction des conditions qui prévalent. Or, ce groupe correspondait à $23 \%$ du total des THQ présents en 2001 .

\section{DES COMPORTEMENTS QUI S'ADAPTENT À LA CONJONCTURE}

Du côté de la demande, les employeurs peuvent adapter leurs stratégies de recrutement à la conjoncture: en période de resserrement, le processus d'embauche sera plus soutenu et les employeurs à la recherche de candidats offriront de meilleurs salaires et conditions de travail; leurs critères relatifs au profil disciplinaire seront assouplis; ils exigeront moins d'années d'expérience préalables, ou un niveau de scolarité plus faible... Cette dernière tactique est fréquente puisqu'en 2001, $27 \%$ des travailleurs qui occupaient un emploi exigeant normalement un grade universitaire n'en détenaient pas un.

À l'opposé, les diplômés à la recherche d'un emploi modifieront eux aussi leur comportement: ils accepteront, par exemple, un emploi à temps partiel, temporaire, peu relié à leur domaine de formation ou exigeant des compétences inférieures aux leurs... Ainsi, en 2003, 15 \% des diplômés de maîtrise de la cohorte de 2001 déclaraient avoir un emploi ayant peu ou pas de rapport avec leur formation et $9 \%$, un emploi à temps partiel.

En résumé, de nombreuses variables fixent les conditions prévalant au cours d'une période donnée sur le marché. Leur diversité est mise en relief dans le schéma ci-dessous. 
FIGURE 1.1

\section{Synthèse des principaux constats relatifs à l'adéquation de l'offre et de la demande}

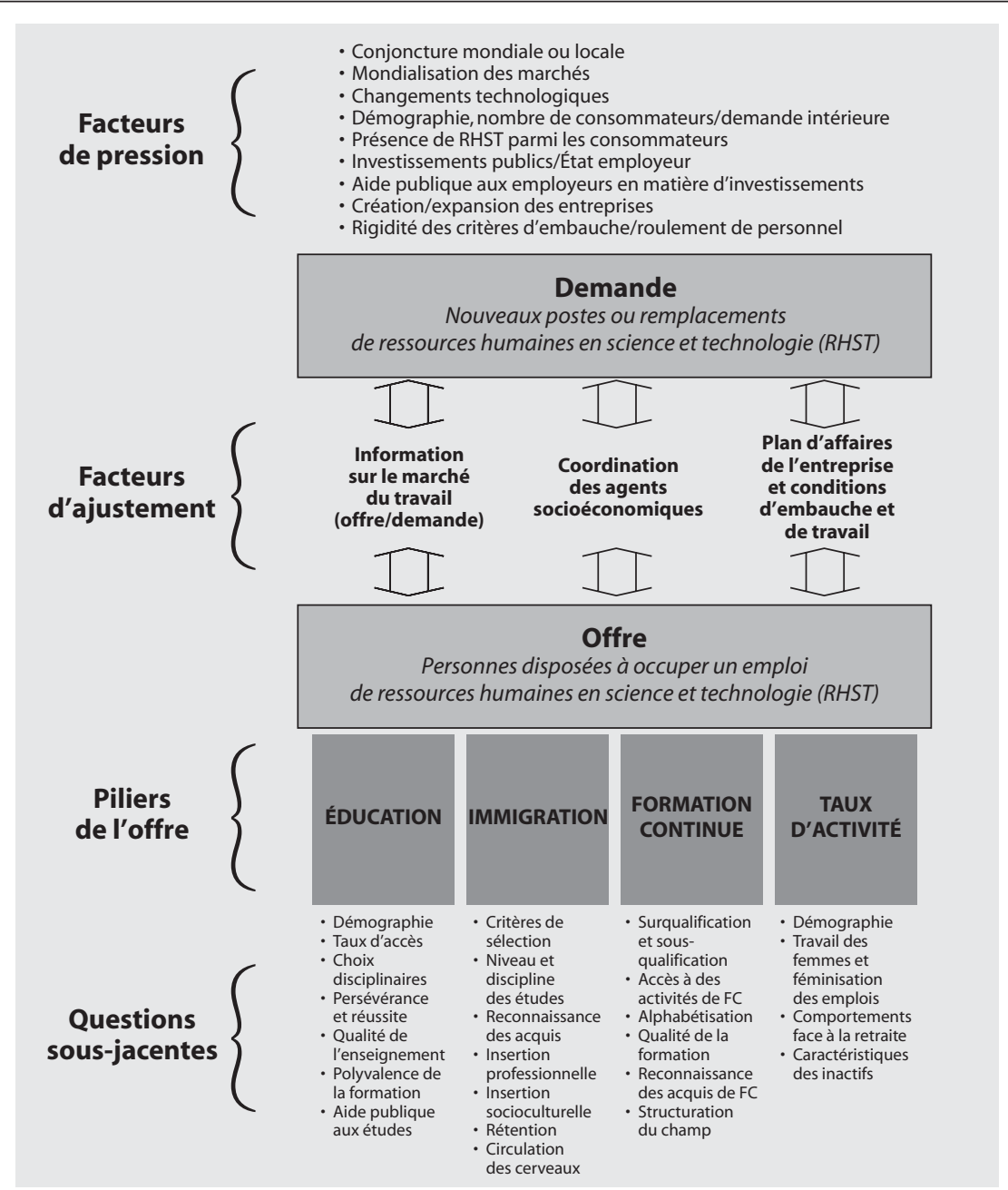

Source: L'avenir de la main-d'ouvre hautement qualifiée. Une question d'ajustements, op. cit., p. 192.

\section{ABSENCE DE PÉNURIE GÉNÉRALISÉE DE THQ À L’HORIZON}

Plusieurs indices ont permis au Conseil de la science et de la technologie de conclure qu'il ne faut pas craindre de pénurie généralisée de THQ à l'échelle du Québec. Parmi ces indices, retenons que de 1990 à 2001, la 
croissance du nombre de diplômés universitaires au travail a été trois fois plus rapide que celle des emplois requérant normalement leur niveau de compétences, ou encore que $27 \%$ des détenteurs d'un grade universitaire n'occupaient pas des emplois conformes à leurs compétences ${ }^{2}$. Ces statistiques révèlent, à n'en pas douter, un phénomène de surqualification d'une partie des diplômés en emploi et donc l'existence d'un réservoir de travailleurs prêts à occuper des emplois mieux adaptés à leur formation. De plus, le taux de chômage en 2001 des titulaires d'un doctorat obtenu en 1999 (6,2\%) est révélateur, même s'il demeure inférieur à celui de l'ensemble de la population active $(8,3 \%)$.

Malgré l'absence de pénurie au niveau agrégé, le Conseil reconnaît que des segments du marché du travail peuvent et pourront éprouver de réelles difficultés d'embauche; l'avènement d'un tel scénario est d'autant plus vraisemblable que les compétences requises sont singulières, que la formation nécessaire est longue, que le secteur est émergent, petit ou soumis à une forte concurrence étrangère...

\section{CHEZ LES DIPLÔMÉS DES CYCLES SUPÉRIEURS, DES DISPARITÉS IMPORTANTES ENTRE DOMAINES DISCIPLINAIRES}

À propos des diplômés des cycles supérieurs, un constat central se dégage: leurs conditions varient énormément, deux ans après la fin de leurs études, selon leur domaine de formation. Les tableaux 1.1 et 1.2 en témoignent.

Ainsi, malgré le niveau somme toute assez agrégé des statistiques apparaissant dans les tableaux, on note des écarts majeurs, aussi bien en ce qui concerne le taux de chômage et les salaires que l'accès à un travail à temps plein ou relié à la formation. L'observation s'applique aux diplômés de maîtrise en 2001 et en 2003, de même qu'à ceux de doctorat en 2001.

Si les variables disponibles pour les conditions de travail sont uniformément positives pour un secteur comme les sciences de l'administration aux deux cycles, il n'en est pas de même des autres secteurs: des taux de chômage relativement faibles peuvent être associés à des salaires ou à des taux d'emploi à temps plein (dans le public ou le parapublic par exemple) inférieurs à la moyenne.

2. Cette statistique est toutefois imparfaite, car elle inclut les détenteurs d'un DEC préuniversitaire, de même que les membres de l'exécutif et des corps législatifs et une catégorie de cadres (les directeurs de société) détenant effectivement un diplôme universitaire. Voir l'avis du Conseil, p.1 et suiv. 
Certains domaines d'études sont très sensibles à la conjoncture: les sciences appliquées, notamment, ont vu le taux de chômage de leurs cohortes de maîtrise tripler en deux ans, le salaire moyen passant sous la moyenne de leur cycle.

À l'aide des seules données disponibles, à savoir celles des cohortes de 1999 interrogées deux ans plus tard, un diplôme de doctorat, plutôt que de maîtrise, n'est pas nécessairement avantageux pour ses détenteurs: le taux de chômage moyen est nettement plus élevé chez les titulaires de doctorat, le salaire moyen est le même et l'emploi à temps plein est moins fréquent. Mais le résultat général ne doit pas être extrapolé à tous les domaines particuliers. Ainsi, les sciences de l'administration et de l'éducation sont des exemples incontestables d'amélioration des conditions de travail. Et il faut demeurer prudents avant de tirer des conclusions puisque 1) les avantages procurés par un doctorat se confirment avec le temps;2) même à court terme, l'emploi occupé est plus relié à la formation; 3) les finissants de maîtrise ont la possibilité de poursuivre leurs études lorsque l'état du marché ne leur convient pas, ce qui permet de réduire leur taux de chômage; pareille option n'existe pas pour les titulaires d'un doctorat, sauf peut-être en sciences de la santé; enfin, 4) des observations portant sur une seule année peuvent être trompeuses.

TABleau 1.1

\section{Situation en 2001 ou 2003 des diplômés de maîtrise promus en 1999 ou 2001}

\begin{tabular}{|l|c|c|c|r|r|r|r|c|}
\hline \multicolumn{1}{|c|}{ Domaines d'études } & \multicolumn{2}{|c|}{ Aux études } & \multicolumn{2}{c|}{$\begin{array}{c}\text { Taux de } \\
\text { chômage } \\
(\%)\end{array}$} & $\begin{array}{c}\text { À temps } \\
\text { plein } \\
(\%)\end{array}$ & $\begin{array}{c}\text { Salaire hebd. brut } \\
\text { moyen } \\
(\$)\end{array}$ & $\begin{array}{c}\text { Relié à la } \\
\text { formation } \\
(\%)\end{array}$ \\
\cline { 2 - 10 } & 2001 & 2003 & 2001 & 2003 & 2003 & 2001 & 2003 & 2003 \\
\hline Sciences de la santé & 25,8 & 28,8 & 3,8 & 3,1 & 89,4 & 917 & 960 & 89,4 \\
\hline Sciences pures & 32,8 & 33,3 & 3,5 & 3,6 & 97,9 & 800 & 846 & 87,0 \\
\hline Sciences appliquées & 16,8 & 22,2 & 3,3 & 10,4 & 96,4 & 1023 & 1014 & 86,9 \\
\hline Sciences humaines & 18,7 & 20,8 & 4,6 & 3,7 & 82,4 & 764 & 828 & 80,8 \\
\hline Lettres & 25,5 & 24,8 & 3,1 & 4,2 & 73,9 & 763 & 744 & 75,5 \\
\hline Droit & 13,2 & 13,8 & 0,0 & 2,7 & 97,3 & 1091 & 1247 & 95,8 \\
\hline Sciences de l'éducation & 8,5 & 15,9 & 1,8 & 4,2 & 87,4 & 954 & 951 & 88,8 \\
\hline $\begin{array}{l}\text { Sciences de } \\
\text { l'administration }\end{array}$ & 3,2 & 4,5 & 3,4 & 4,0 & 98,1 & 1200 & 1234 & 87,2 \\
\hline Arts & 17,9 & 23,9 & 10,8 & 5,9 & 68,4 & 707 & 670 & 63,1 \\
\hline Études plurisectorielles & 14,1 & 14,3 & 4,9 & 2,5 & 94,9 & 844 & 894 & 88,0 \\
\hline Ensemble du Québec & 15,2 & 17,3 & 3,7 & 4,6 & 91,2 & 980 & 1021 & 85,4 \\
\hline
\end{tabular}

Source: MEQ, La relance à l'université. 
TABLEAU 1.2

Situation en janvier 2001 des titulaires d'un doctorat de la promotion 1999 selon le domaine de formation

\begin{tabular}{|c|c|c|c|c|c|c|}
\hline \multirow[b]{2}{*}{ Famille de programmes } & \multirow[b]{2}{*}{$\begin{array}{c}\text { Aux études } \\
(\%)\end{array}$} & \multirow[b]{2}{*}{$\begin{array}{l}\text { Inactifs } \\
(\%)\end{array}$} & \multirow[b]{2}{*}{$\begin{array}{c}\text { Taux de } \\
\text { chômage } \\
(\%)\end{array}$} & \multicolumn{3}{|c|}{ Personnes en emploi } \\
\hline & & & & $\begin{array}{c}\text { À temps } \\
\text { plein } \\
(\%)\end{array}$ & $\begin{array}{c}\text { Salaire hebd. } \\
(\%)\end{array}$ & $\begin{array}{c}\text { Relié à la } \\
\text { formation } \\
(\%)\end{array}$ \\
\hline \multicolumn{7}{|l|}{ Domaine de formation } \\
\hline Sciences de la santé & 17,1 & 2,9 & 3,6 & 96,3 & 1054 & 94,2 \\
\hline Sciences pures & 2,2 & 0,0 & 9,2 & 98,7 & 948 & 92,3 \\
\hline Sciences appliquées & 1,7 & 1,7 & 8,1 & 94,1 & 1008 & 93,8 \\
\hline Sciences humaines & 5,1 & 6,8 & 5,8 & 79,5 & 940 & 94,0 \\
\hline Lettres & 2,3 & 6,8 & 7,5 & 89,2 & 883 & 93,9 \\
\hline Droit & 0,0 & 0,0 & 0,0 & 100,0 & 731 & 100,0 \\
\hline Sciences de léducation & 2,9 & 0,0 & 3,0 & 81,3 & 1184 & 88,5 \\
\hline Sciences de l'administration & 0,0 & 0,0 & 0,0 & 88,9 & 1454 & 93,8 \\
\hline Arts & 0,0 & 22,2 & 0,0 & 28,6 & 837 & 100,0 \\
\hline Études plurisectorielles & 0,0 & 0,0 & 0,0 & 83,3 & 848 & 100,0 \\
\hline Tous & 4,8 & 3,7 & 6,2 & 88,3 & 988 & 93,5 \\
\hline
\end{tabular}

Source: MEQ, La relance à l'université, placement des personnes diplômées en 1999 pendant la semaine du 14 au 21 janvier 2001, Québec, 2002.

\section{LES LEÇONS À TIRER}

Quelles leçons peut-on tirer de ces observations? Le Conseil en énonce plusieurs destinées à l'ensemble des acteurs. Nous retiendrons ici celles qui concernent plus particulièrement les étudiants et les universités.

- Plus un étudiant se soucie des conditions de travail qui lui seront offertes, plus il tirera avantage à s'informer tôt, puis régulièrement, de l'évolution du marché. Encore faut-il que le gouvernement diffuse très largement une information juste et fine sur l'état actuel et prévisible du marché et de ses composantes.

- Les programmes de formation initiale devront être revus de manière à doter les futurs diplômés d'une solide formation générale et d'une polyvalence qui maximisera la probabilité de décrocher un emploi conforme à leurs compétences. De plus, la formation doctorale doit tenir compte du fait que les postes d'universitaires représentent moins de $40 \%$ des possibilités d'emploi pour les finissants. Enfin, 
le Conseil invite les universités et les organismes sectoriels d'intervention, notamment les comités sectoriels de main-d'œuvre, à collaborer plus étroitement que jamais afin de stabiliser l'évolution du marché et de réduire les temps d'attente en matière de recrutement.

- Le Conseil propose que le taux d'obtention d'un doctorat au Québec, de 1,0\% qu'il était en 2001, soit haussé à 1,3\% d'ici 2010. Certes, les statistiques dévoilées dans l'avis n'appuient pas cette proposition. Mais il s'agit d'un choix de société qui se justifie par l'effet d'entraînement que les diplômés exercent sur le développement économique et le progrès social. Le Conseil ne suggère pas de cible au niveau de la maîtrise, le taux actuel (7,3\% en 2001) se comparant avantageusement à celui des pays usuels de référence.

- Les diplômés des cycles supérieurs doivent aussi s'attendre, beaucoup plus que maintenant, à consacrer une partie importante de leurs occupations professionnelles à la formation continue, d'une part, à cause des exigences de l'économie du savoir et, d'autre part, à cause du retard pris par le Québec en cette matière.

- Le Conseil recommande en outre de stimuler la création d'emplois dans certains secteurs, notamment pour les étudiants des cycles supérieurs en sciences pures et appliquées. Les politiques et stratégies gouvernementales visant à soutenir la recherche et l'innovation devraient être de puissants leviers pour y parvenir.

Enfin, pour clore cette réflexion, je rappellerai que le Conseil prône un rapprochement plus étroit entre la communauté scientifique et la population en général. Il y consacre d'ailleurs d'importantes sections de son nouveau rapport de conjoncture ${ }^{3}$, lesquelles cherchent à en justifier le bien-fondé et à suggérer des façons de le concrétiser. Or les étudiants des cycles supérieurs d'aujourd'hui forment déjà et surtout formeront l'essentiel de cette communauté scientifique. Ils devront à ce titre faire preuve des talents requis pour un dialogue efficace: sens de l'écoute, communication claire et vulgarisée, prise en compte du message des interlocuteurs... Mais c'est là un autre sujet.

3. Conseil de la science et de la technologie, La culture scientifique et technique, une interface entre les sciences, la technologie et la société, 2004. 



\title{
Chapitre
}

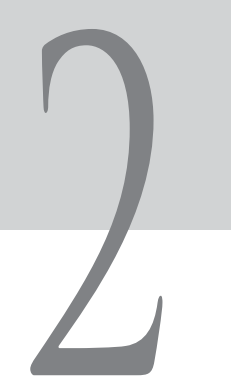

\section{QU'ADVIENT-IL DES DIPLÔMÉS DES CYCLES SUPÉRIEURS?}

\author{
MM. Pierre Michel et François Girard ${ }^{1}$ \\ Direction de la recherche, des statistiques \\ et des indicateurs (MEQ)
}

L'économie du savoir est un pilier de l'activité économique du Québec lui permettant de suivre le rythme effréné d'un système économique encore plus complexe et englobant que jamais depuis les dernières décennies. Elle représente aussi un potentiel de développement durable et une ressource importante pour améliorer les conditions de vie de ses habitants. Bien que la connaissance semble être un réservoir inépuisable de ressources, d'idées, de solutions, etc., il faut aussi se doter de moyens pour pouvoir les utiliser. Autrement dit, la réalisation concrète de cette économie du savoir passe par la contribution (par exemple, l'emploi) de ceux qui possèdent la connaissance. La fin des études correspond, justement, à l'un des moments qui contribuent d'emblée à la réalisation des savoirs du système scolaire québécois.

1. MM. Pierre Michel et François Girard sont les responsables des enquêtes d'insertion professionnelle Relance au sein du MEQ, Direction de la recherche, des statistiques et des indicateurs (DRSI), Services des études économiques et démographiques. 
Dans ce chapitre, nous reprenons les principaux jalons de la présentation des enquêtes La relance à l'université, 2001 et 2003. Plusieurs thèmes furent abordés, soit les objectifs des enquêtes Relance à l'université, 2001 et 2003, la méthodologie et le recensement, certaines caractéristiques des personnes diplômées des cycles supérieurs ainsi que l'emploi chez ces personnes.

\section{LES OBJECTIFS DES ENQUÊTES RELANCE À L'UNIVERSITÉ, 2001 ET 2003}

Ces enquêtes permettent de décrire et de faire connaître la situation des diplômés universitaires environ 20 mois après l'obtention de leur diplôme. Elles comblent un besoin d'information fiable et actualisée sur la situation d'emploi des personnes diplômées.

\section{LA MÉTHODOLOGIE ET LE RECENSEMENT}

La base de la population cible de ces enquêtes provient du fichier Gestion des données sur l'effectif universitaire (GEDU). Elle est étayée par la mise à jour des coordonnées des personnes diplômées par le Bureau du registraire de chacune des universités québécoises. Ce sont 898 répondants titulaires d'un doctorat ${ }^{2}$ ayant obtenu leur diplôme en 1999, ainsi que 6429 personnes titulaires d'une maîtrise ${ }^{3}$, qui ont pu être interrogés.

\section{QUELQUES CARACTÉRISTIQUES DES PERSONNES DIPLÔMÉES}

\section{Maîtrise}

D'abord, il faut signaler qu'il n'existe aucun écart important entre le fait d'être un homme ou une femme, et ce, en ce qui concerne les caractéristiques des titulaires d'une maîtrise en 2001. Parmi ces titulaires, 76,2\% ont déclaré être en emploi pour la semaine de référence du 19 au 25 janvier 2003. Le tableau 2.1 permet également de relever qu'à cette composante de la population active (être en emploi) s'ajoutent 3,7\% des titulaires qui affirmaient être à la recherche d'un emploi. Le taux de chômage était donc, selon le rapport diplômés/population active, de 4,6\%. Enfin, parmi l'ensemble des titulaires d'une maîtrise en 2001, 17,3\% ont indiqué être aux études pendant cette même période de référence.

\footnotetext{
2. Le nombre de personnes diplômées correspond à un taux de réponse de $63 \%$ en 2001 .

3. Le taux de réponse au moment de l'enquête de 2003 était de 69,1\%.
} 
TABleau 2.1

Situation en $\mathbf{2 0 0 3}$ des titulaires d'une maîtrise de 2001 (selon le sexe) (semaine de référence du 19 au 25 janvier 2003)

\begin{tabular}{|c|c|c|c|c|c|}
\hline Type de diplôme & Sexe & En emploi & $\begin{array}{c}\text { À la recherche } \\
\text { d'un emploi } \\
(\%)\end{array}$ & $\begin{array}{c}\text { Aux études } \\
(\%)\end{array}$ & $\begin{array}{c}\text { Taux de chômage } \\
(\%)\end{array}$ \\
\hline \multirow{3}{*}{ Maîtrise } & Masculin & 75,2 & 4,2 & 19,5 & 5,3 \\
\cline { 2 - 6 } & Féminin & 77,0 & 3,3 & 15,4 & 4,1 \\
\cline { 2 - 6 } & Ensemble & 76,2 & 3,7 & 17,3 & 4,6 \\
\hline
\end{tabular}

Source: La relance à l'université, 2003.

Par ailleurs, nous pouvons constater une variation de l'insertion à l'emploi selon le domaine d'études des titulaires d'une maitrise (tableau 2.2). En effet, la proportion la plus élevée d'individus en emploi est associée aux Sciences de l'administration (89,7\%); suivi du Droit (83,9\%) et des Études plurisectorielles (80,6\%). De plus, c'est en Sciences appliquées et en Arts que se retrouve la proportion la plus élevée de personnes diplômées à la recherche d'un emploi, soit respectivement $7,9 \%$ et $4,2 \%$. Notons également au passage que c'est au sein de ces

\section{TABLEAU 2.2}

\section{Situation en $\mathbf{2 0 0 3}$ des titulaires d'une maîtrise de 2001} (selon le domaine d'études)

\begin{tabular}{|l|c|c|c|c|c|}
\hline \multicolumn{1}{|c|}{ Domaine d'études } & $\begin{array}{c}\text { Personnes } \\
\text { diplômées } \\
\text { visées par } \\
\text { l'enquête } \\
\mathrm{N}\end{array}$ & En emploi & $\begin{array}{c}\text { À la recherche } \\
\text { d'un emploi }\end{array}$ & Aux études & $\begin{array}{c}\text { Taux de } \\
\text { chômage }\end{array}$ \\
\hline Sciences de l'administration & 2078 & 89,7 & 3,7 & 4,5 & 4,0 \\
\hline Droit & 137 & 83,9 & 2,3 & 13,8 & 2,7 \\
\hline Études plurisectorielles & 130 & 80,6 & 2,0 & 14,3 & 2,5 \\
\hline Sciences de l'éducation & 316 & 76,6 & 3,3 & 15,9 & 4,2 \\
\hline Sciences humaines & 1433 & 72,8 & 2,8 & 20,8 & 3,7 \\
\hline Sciences appliquées & 882 & 67,5 & 7,9 & 22,2 & 10,4 \\
\hline Sciences de la santé & 536 & 67,2 & 2,1 & 28,8 & 3,1 \\
\hline Arts & 202 & 66,9 & 4,2 & 23,9 & 5,9 \\
\hline Lettres & 276 & 65,7 & 2,9 & 24,8 & 4,2 \\
\hline Sciences pures & 439 & 62,0 & 2,3 & 33,3 & 3,6 \\
\hline Ensemble du Québec & 6429 & 76,2 & 3,7 & 17,3 & 4,6 \\
\hline
\end{tabular}

Source: La relance à l'université, 2003. 
mêmes disciplines que l'on retrouve le plus haut taux de chômage, soit $10,4 \%$ en Sciences appliquées et 5,9\% en Arts. Enfin, c'est en Sciences pures $(33,3 \%)$ et en Sciences de la santé $(28,8 \%)$ que se trouve la plus forte proportion de personnes titulaires d'une maîtrise déclarant avoir été aux études pendant la période du 19 au 25 janvier 2003.

\section{Doctorat}

Parmi les titulaires d'un doctorat qui furent interrogés, $85,9 \%$ ont déclaré être en emploi au cours de la semaine de référence de l'enquête, soit du 14 au 20 janvier 2001 (tableau 2.3). De plus, se chercher un emploi était, pendant cette période, la préoccupation principale chez $5,6 \%$ d'entre eux. Le rapport, exprimé en pourcentage, entre le nombre de personnes diplômées à la recherche d'un emploi et l'ensemble de la population active (c.-à-d. le taux de chômage) était alors de 6,2\%. Enfin, $4,8 \%$ des titulaires d'un doctorat affirmaient être aux études entre le 14 et le 20 janvier 2001.

TABLEAU 2.3

Situation en 2001 des titulaires d'un doctorat de 1999 (selon le sexe)

\begin{tabular}{|c|c|c|c|c|c|c|}
\hline $\begin{array}{c}\text { Type de } \\
\text { diplôme }\end{array}$ & Sexe & $\begin{array}{c}\text { Personnes } \\
\text { diplômées } \\
\text { visées par } \\
\text { l'enquête } \\
\text { N }\end{array}$ & En emploi & $\begin{array}{c}\text { À la recherche } \\
\text { d'un emploi }\end{array}$ & Aux études & $\begin{array}{c}\text { Taux de } \\
\text { chômage }\end{array}$ \\
\hline \multirow{4}{*}{ Doctorat } & Masculin & 548 & 86,4 & 6,8 & 4,4 & 7,3 \\
\cline { 2 - 7 } & Féminin & 350 & 85,0 & 4,0 & 5,3 & 4,5 \\
\cline { 2 - 7 } & Ensemble & 898 & 85,9 & 5,6 & 4,8 & 6,2 \\
\hline
\end{tabular}

Source: La relance à l'université, 2001.

De plus, il faut remarquer que certains domaines d'études partagent les mêmes caractéristiques. En effet, les titulaires d'un doctorat en Droit, en Sciences de l'administration et en Études plurisectorielles (tableau 2.4) retiennent particulièrement l'attention lorsqu'on s'attarde à la fois au plus haut pourcentage d'individus en emploi (soit $100 \%$ dans les trois domaines), au taux de chômage ( $0,0 \%$ pour les trois) et à la proportion de ces titulaires affirmant avoir été aux études pendant la période de référence ( $0,0 \%$ pour les trois). À cette liste s'ajoutent $94,1 \%$ des personnes diplômées en emploi dans le domaine des Sciences de l'éducation. Parmi ces derniers, 2,9\% étaient aux études entre le 14 et le 20 janvier 2001. 
TABLEAU 2.4

Situation en 2001 des titulaires d'un doctorat de 1999

(selon le domaine d'études)

\begin{tabular}{|l|c|c|c|c|c|}
\hline \multicolumn{1}{|c|}{ Domaine d'études } & $\begin{array}{c}\text { Personnes } \\
\text { diplômées } \\
\text { visées par } \\
\text { l'enquête } \\
\text { N }\end{array}$ & En emploi & $\begin{array}{c}\text { À la recherche } \\
\text { d'un emploi }\end{array}$ & Aux études & $\begin{array}{c}\text { Taux de } \\
\text { chômage }\end{array}$ \\
\hline Droit & 6 & 100,0 & 0,0 & 0,0 & 0,0 \\
\hline Sciences de l'administration & 28 & 100,0 & 0,0 & 0,0 & 0,0 \\
\hline Études plurisectorielles & 9 & 100,0 & 0,0 & 0,0 & 0,0 \\
\hline Sciences de l'éducation & 50 & 94,1 & 2,0 & 2,9 & 3,0 \\
\hline Sciences pures & 157 & 88,8 & 9,0 & 2,2 & 9,2 \\
\hline Sciences appliquées & 184 & 88,7 & 7,8 & 1,7 & 8,1 \\
\hline Lettres & 69 & 84,1 & 6,8 & 2,3 & 7,5 \\
\hline Sciences humaines & 255 & 83,0 & 5,1 & 5,1 & 5,8 \\
\hline Arts & 12 & 77,8 & 0,0 & 0,0 & 0,0 \\
\hline Sciences de la santé & 128 & 77,1 & 2,9 & 17,1 & 3,6 \\
\hline Ensemble du Québec & 898 & 85,9 & 5,6 & 4,8 & 6,2 \\
\hline
\end{tabular}

Source: La relance à l'université, 2001.

\section{L'EMPLOI CHEZ LES PERSONNES DIPLÔMÉES}

\section{Les moyens pour décrocher un emploi ${ }^{4}$}

\section{TABLEAU 2.5}

Situation en 2003 des titulaires d'une maîtrise de 2001 (recherche d'emploi)

\begin{tabular}{|l|c|}
\hline \multicolumn{1}{|c|}{ Titulaires qui ont obtenu un premier emploi salarié d'importance à la fin de leurs études } & Titulaires d'une maitrise \\
\hline Par des contacts directs et actifs de la part du titulaire auprès des employeurs (\%). & 32,3 \\
\hline Par un service, des contacts ou des annonces trouvés à l'extérieur de l'université (\%). & 30,5 \\
\hline Le titulaire a été approché par un employeur (\%). & 9,7 \\
\hline À la suite d'un stage en cours d'études (\%). & 8,8 \\
\hline Services de placement à l'université (\%). & 8,2 \\
\hline Par lintermédiaire de professeurs, de responsables de stages à l'université (\%). & 6,4 \\
\hline Par des collègues d'études (\%). & 2,1 \\
\hline Par une journée carrière à l'université (\%). & 1,4 \\
\hline Autres moyens, ressources ou stratégie (\%). & 0,5 \\
\hline
\end{tabular}

Source: La relance à l’université, 2003.

4. Données disponibles uniquement pour les titulaires d'une maîtrise en 2001. 
Parmi l'ensemble des personnes titulaires d'une maîtrise en 2001 qui ont cherché un emploi après l'obtention de leur diplôme, 32,3\% affirment avoir obtenu un premier emploi d'importance à partir de contacts directs et actifs auprès des employeurs ; 30,5\% ont eu recours à un service, à des contacts ou à des annonces trouvées à l'extérieur de l'université. C'est dans une proportion de 9,7\% qu'ils furent approchés par les employeurs. En ce qui concerne les autres moyens utilisés, on note que $8,8 \%$ ont décroché un emploi à la suite d'un stage en cours d'études et que $8,2 \%$ ont eu recours aux services de placement à l'université.

\section{La durée de la recherche d'emploi}

La durée de recherche d'un emploi chez les personnes diplômées varie selon le domaine d'études et le type de diplôme. Par exemple, chez les titulaires d'une maîtrise, le nombre de semaines de recherche peut varier entre 8 (en Sciences de la santé) et 22 semaines (en Arts) après l'obtention du diplôme (en 2001). Pour ce qui est des personnes titulaires d'un doctorat en Sciences de l'éducation et en Arts (en 1999), elles ont dû chercher respectivement en moyenne 10 et 52 semaines pour se trouver un emploi.

Les données disponibles sur la durée de recherche d'un emploi permettent également de constater, à titre indicatif, qu'une personne titulaire d'une maîtrise en Sciences humaines (en 2001) a dû chercher le même nombre de semaines (soit 14) qu'une personne titulaire du doctorat (en 1999) dans le même domaine d'études. On peut faire la même observation entre une personne titulaire de la maîtrise en Sciences pures (soit 10 semaines) et une personne titulaire d'un doctorat (soit 11 semaines) dans le même domaine.

\section{L'emploi à temps plein}

Parmi l'ensemble des personnes diplômées de la maîtrise qui étaient en emploi (76,2\%), 91,2\% d'entre eux avaient un emploi à temps plein (voir le tableau 2.6). Pour cette majorité en emploi, il importe de souligner que les hommes $(95,4 \%)$ travaillent à temps plein ${ }^{5}$ dans une plus forte proportion que les femmes (87,9\%). Par ailleurs, $85,4 \%$ des titulaires interrogés affirmaient que leur emploi à temps plein avait un rapport avec

5. Parmi les répondants à l'enquête titulaires d'une maîtrise, il y avait 2996 hommes et 3433 femmes. 
la formation qu'ils avaient reçue. Le sentiment d'utilité des connaissances et des expériences acquises dans l'exercice de leur travail est aussi un élément qui fut relevé par une forte majorité des titulaires d'une maitrise en emploi (entre 92,5\% et 99,4\% selon le domaine d'études).

TABLEAU 2.6

Situation en 2003 des titulaires d'une maîtrise de 2001 (caractéristiques de l'emploi)

\begin{tabular}{|c|c|c|c|c|c|c|}
\hline \multirow[b]{2}{*}{$\begin{array}{l}\text { Type de } \\
\text { diplôme }\end{array}$} & \multirow[b]{2}{*}{ Sexe } & \multirow{2}{*}{$\begin{array}{l}\text { Personnes } \\
\text { diplômées } \\
\text { visées par } \\
\text { l'enquête } \\
\text { N }\end{array}$} & \multirow{2}{*}{$\begin{array}{l}\text { En emploi } \\
\\
(\%)\end{array}$} & \multicolumn{3}{|c|}{ L'emploi à temps plein et ses caractéristiques } \\
\hline & & & & $\begin{array}{c}\text { À temps plein } \\
(\%)\end{array}$ & $\begin{array}{c}\text { Durée de } \\
\text { recherche } \\
\text { (sem.) }\end{array}$ & $\begin{array}{c}\begin{array}{c}\text { Relié à la } \\
\text { formation }\end{array} \\
(\%)\end{array}$ \\
\hline \multirow{3}{*}{ Maîtrise } & Masculin & 2996 & 75,2 & 95,4 & 13 & 85,0 \\
\hline & Féminin & 3433 & 77,0 & 87,9 & 13 & 85,7 \\
\hline & Ensemble & 6429 & 76,2 & 91,2 & 13 & 85,4 \\
\hline
\end{tabular}

Source: La relance à l'université, 2003.

Être en emploi à temps plein entre le 14 et le 20 janvier 2001 était, dans une proportion de $88,3 \%$, la situation des titulaires d'un doctorat en 1999 (tableau 2.7). D’ailleurs, les hommes (92,2\%) semblent être représentés dans une plus grande proportion que les femmes $(82,4 \%)$ pour un emploi à temps plein (plus de 30 heures par semaine). Il faut également signaler que c'est une forte majorité $(93,5 \%)$ de titulaires d'un doctorat qui ont souligné le fait qu'il y avait un rapport entre leur emploi à temps plein et leur formation.

TABleau 2.7

\section{Situation en 2001 des titulaires d'un doctorat de 1999}

(caractéristiques de l'emploi)

\begin{tabular}{|c|c|c|c|c|c|c|}
\hline \multirow{3}{*}{$\begin{array}{l}\text { Type de } \\
\text { diplôme }\end{array}$} & \multirow{3}{*}{ Sexe } & \multirow{3}{*}{$\begin{array}{c}\text { Personnes } \\
\text { diplômées } \\
\text { visées par } \\
\text { l'enquête } \\
\text { N }\end{array}$} & \multirow{3}{*}{$\begin{array}{c}\text { En emploi } \\
\\
(\%)\end{array}$} & \multicolumn{3}{|c|}{ L'emploi à temps plein et ses caractéristiques } \\
\hline & & & & À temps plein & $\begin{array}{l}\text { Durée de } \\
\text { recherche }\end{array}$ & $\begin{array}{l}\text { Reliéà la } \\
\text { formation }\end{array}$ \\
\hline & & & & $(\%)$ & (sem.) & $(\%)$ \\
\hline \multirow{3}{*}{ Doctorat } & Masculin & 548 & 86,4 & 92,2 & 13 & 92,2 \\
\hline & Féminin & 350 & 85,0 & 82,4 & 18 & 95,6 \\
\hline & Ensemble & 898 & 85,9 & 88,3 & 15 & 93,5 \\
\hline
\end{tabular}

Source: La relance à l'université, 2001. 


\section{L'emploi permanent lié à la formation}

On remarque d'abord une différence quant à la proportion d'hommes $(87,5 \%)$ et de femmes $(77,1 \%)$ titulaires d'une maîtrise qui affirmaient avoir un emploi permanent relié à leur formation. L'emploi temporaire caractérisait ainsi la situation «en emploi» de 22,9\% des femmes titulaires d'une maîtrise.

TABLEAU 2.8

Situation en 2003 des titulaires d'une maîtrise de 2001 (statut d'emploi)

\begin{tabular}{|c|c|c|c|c|}
\hline \multirow{3}{*}{ Permanence liée à la formation } & \multicolumn{4}{|c|}{ Sexe } \\
\hline & \multicolumn{2}{|c|}{ Hommes } & \multicolumn{2}{|c|}{ Femmes } \\
\hline & $\mathrm{N}$ & $(\%)$ & $\mathrm{N}$ & $(\%)$ \\
\hline Emploi permanent & 1004 & 87,5 & 1157 & 77,1 \\
\hline Emploi temporaire & 143 & 12,5 & 343 & 22,9 \\
\hline Total & 1147 & 100,0 & 1500 & 100,0 \\
\hline
\end{tabular}

Source: La relance à l'université, 2003.

L'écart semble moins important entre les hommes $(67,7 \%)$ et les femmes $(59,1 \%)$ titulaires d'un doctorat qui avaient un emploi permanent lié à leur formation. Par ailleurs, les personnes diplômées qui occupaient un emploi temporaire étaient représentées dans une proportion de $40,9 \%$ chez les femmes et de $32,3 \%$ chez les hommes.

TABLEAU 2.9

Situation en 2001 des titulaires d'un doctorat de 1999 (statut d'emploi)

\begin{tabular}{|c|c|c|c|c|}
\hline \multirow{3}{*}{ Permanence liée à la formation } & \multicolumn{4}{|c|}{ Sexe } \\
\hline & \multicolumn{2}{|c|}{ Hommes } & \multicolumn{2}{|c|}{ Femmes } \\
\hline & $\mathrm{N}$ & $(\%)$ & $\mathrm{N}$ & $(\%)$ \\
\hline Emploi permanent & 168 & 67,7 & 101 & 59,1 \\
\hline Emploi temporaire & 80 & 32,3 & 70 & 40,9 \\
\hline Total & 248 & 100,0 & 171 & 100,0 \\
\hline
\end{tabular}

Source: La relance à l'université, 2001. 


\section{Taille des entreprises qui emploient les personnes diplômées}

Les employeurs dont la taille de l'entreprise était au moins 500 employés avaient procuré un emploi à plus de la moitié $(56,4 \%)$ des titulaires d'une maîtrise entre le 19 et le 25 janvier 2003. Ceux et celles qui travaillaient pour une entreprise moyenne (entre 100 et 499 employés) représentaient $14,8 \%$, comparativement à $12,7 \%$ qui occupaient un emploi au sein d'une petite entreprise constituée de 1 à 25 employés.

TABleau 2.10

Situation en $\mathbf{2 0 0 3}$ des titulaires d'une maîtrise de 2001

(taille des entreprises)

\begin{tabular}{|l|c|c|}
\hline \multicolumn{1}{|c|}{ Taille des entreprises } & $\mathrm{N}$ & $(\%)$ \\
\hline 1 à 25 employés & 336 & 12,7 \\
\hline 26 à 99 employés & 238 & 9,0 \\
\hline 100 à 499 employés & 391 & 14,8 \\
\hline 500 employés et plus & 1493 & 56,4 \\
\hline Total & 2647 & 100,0 \\
\hline
\end{tabular}

Source: La relance à l'université, 2003.

Les grandes entreprises (500 employés et plus) constituent également la principale porte d'entrée sur le marché du travail pour $60,2 \%$ des titulaires d'un doctorat. Quant à la taille des entreprises qui engageaient $21,5 \%$ de ces autres personnes possédant un doctorat en emploi, elle se situait entre 100 à 499 employés.

TABLEAU 2.11

Situation en 2001 des titulaires d'un doctorat de 1999

(taille des entreprises)

\begin{tabular}{|l|c|c|}
\hline \multicolumn{1}{|c|}{ Taille des entreprises } & $\mathrm{N}$ & $(\%)$ \\
\hline 1 à 25 employés & 31 & 8,4 \\
\hline 26 à 99 employés & 36 & 9,8 \\
\hline 100 à 499 employés & 79 & 21,5 \\
\hline 500 employés et plus & 221 & 60,2 \\
\hline Total & 367 & 100,0 \\
\hline
\end{tabular}

Source: La relance à l'université, 2001. 


\section{Raisons de ne pas être en emploi}

La principale raison évoquée par 53,5\% des titulaires d'une maîtrise qui n'étaient pas en emploi pendant la semaine du 19 au 25 janvier 2003 est le manque de postes disponibles (région visée, domaine visé, conjoncture économique, etc.).

TABLEAU 2.12

\section{Situation en 2003 des titulaires d'une maîtrise de 2001 (chômage)}

\begin{tabular}{|l|c|c|}
\hline \multicolumn{1}{|c|}{ Raisons de ne pas être en emploi } & $\mathrm{N}$ & $(\%)$ \\
\hline $\begin{array}{l}\text { Vous faisiez face à un manque de postes disponibles (région visée, domaine visé, } \\
\text { conjoncture économique, etc.). }\end{array}$ & 121 & 53,5 \\
\hline Autres raisons. & 32 & 14,2 \\
\hline Congédiement, fin de contrat, fermeture d'entreprise. & 24 & 10,6 \\
\hline Vous étiez aux études. & 12 & 5,3 \\
\hline Vous n'aviez pas l'expérience requise. & 12 & 5,3 \\
\hline $\begin{array}{l}\text { Vous étiez temporairement dans l'incapacité de travailler (problèmes de santé, raisons } \\
\text { familiales, congé de maternité, etc.). }\end{array}$ & 8 & 3,5 \\
\hline Vous n'aviez pas les qualifications requises pour exercer. & 7 & 3,1 \\
\hline Vous aviez un emploi mais n'aviez pas commencé à travailler. & 4 & 1,8 \\
\hline Votre âge causait problème. & 4 & 1,8 \\
\hline Vous ne connaissiez pas assez bien le français. & 2 & 0,9 \\
\hline Total & 226 & 100,0 \\
\hline
\end{tabular}

Source: La relance à l'université, 2003.

C'est également ce qu'ont répondu $54,8 \%$ des titulaires d'un doctorat qui ne travaillaient pas entre le 14 et le 20 janvier 2001 (voir le tableau 2.13). Le congédiement, les fins de contrats et les fermetures d'entreprises sont aussi des situations auxquelles ont fait face 10,6\% des titulaires d'une maîtrise et $16,7 \%$ des titulaires d'un doctorat. 
TABLEAU 2.13

Situation en 2001 des titulaires d'un doctorat de 1999 (chômage)

\begin{tabular}{|l|c|c|}
\hline \multicolumn{1}{|c|}{ Raisons de ne pas être en emploi } & $\mathrm{N}$ & $(\%)$ \\
\hline $\begin{array}{l}\text { Vous faisiez face à un manque de postes disponibles (région visée, domaine visé, } \\
\text { conjoncture économique, etc.). }\end{array}$ & 23 & 54,8 \\
\hline Congédiement, fin de contrat, fermeture d'entreprise. & 7 & 16,7 \\
\hline Autres raisons. & 5 & 11,9 \\
\hline Vous n'aviez pas l'expérience requise. & 4 & 9,5 \\
\hline Vous étiez en stage dans le but d'obtenir un droit de pratique. & 2 & 4,8 \\
\hline Vous aviez un emploi mais n'aviez pas commencé à travailler. & 1 & 2,4 \\
\hline Total & 42 & 100,0 \\
\hline
\end{tabular}

Source: La relance à l'université, 2001.

\section{CONCLUSION}

Intégrer le marché de l'emploi est un processus relativement complexe. Afin de bien comprendre ce processus, il faut nécessairement identifier et décrire des situations clés (être à la recherche d'un emploi, être en emploi, en emploi à temps plein, etc.), tout en mettant en relations certains indicateurs (la population active, le taux d'emploi, le taux de chômage, le domaine d'études, etc.) pertinents à l'analyse de la situation des personnes diplômées.

Les résultats des enquêtes Relance à l'université, 2001 et 2003, permettent d'alimenter les connaissances quant au processus d'insertion dans le marché du travail des personnes diplômées des cycles supérieures. Connaître certaines des caractéristiques et des conditions d'insertion professionnelle des titulaires d'une maîtrise ou d'un doctorat contribue à la mise en application des savoirs de ces personnes diplômées. 


\section{ANNEXE - DÉFINITIONS 6}

\section{Personnes diplômées visées par l'enquête}

Sont considérées comme des «personnes diplômées visées par l'enquête» toutes les personnes titulaires d'une maîtrise ou d'un doctorat qui ont reçu leur diplôme au cours de l'année civile précédant de deux ans l'année de l'enquête.

\section{Taux de réponse}

Rapport, exprimé en pourcentage, entre le nombre de personnes diplômées visées par l'enquête qui ont répondu au questionnaire et l'ensemble des personnes diplômées visées par l'enquête.

\section{En emploi ${ }^{7}$}

Sont dites «en emploi» les personnes diplômées visées par l'enquête qui ont déclaré travailler à temps plein à leur compte ou pour autrui, sans étudier à temps plein.

\section{À la recherche d'un emploi}

Sont dites «à la recherche d'un emploi» les personnes diplômées visées par l'enquête qui ont déclaré se chercher un emploi, sans être aux études ni être déjà en emploi.

\section{Aux études}

Sont dites «aux études » les personnes diplômées visées par l'enquête qui ont déclaré soit étudier à temps plein, soit étudier à temps partiel sans avoir d'emploi.

6. Les définitions sont extraites de La relance à l'université - 2003, La situation d'emploi des personnes diplômées, Enquête de 2003, Direction de la recherche, des statistiques et des indicateurs, Ministère de l'Éducation du Québec, 2004.

7. Les personnes qui ont déclaré être aux études à temps plein et travailler à temps plein sont considérées comme étant «aux études» en 2003, alors qu'elles étaient considérées comme étant «en emploi» en 2001. Cette modification réduit un peu (d'environ 2,2\%) la proportion de personnes «en emploi» au profit de la proportion de personnes «aux études». Les définitions des indicateurs «en emploi» et «aux études» présentées ici concernent donc les résultats de 2003, tandis que cette note permettra de comprendre les résultats de 2001. 


\section{Personnes inactives}

Sont considérées comme des «personnes inactives » les personnes diplômées visées par l'enquête qui ont déclaré ne pas avoir d'emploi, ne pas en chercher et ne pas être aux études.

\section{À temps plein}

Sont dites «à temps plein» les personnes diplômées en emploi qui travaillent, de façon générale, 30 heures ou plus par semaine.

\section{À temps partiel}

Sont dites «à temps partiel» les personnes diplômées en emploi qui travaillent, de façon générale, moins de 30 heures par semaine. La proportion des personnes diplômées qui travaillent à temps partiel s'obtient en soustrayant la proportion des personnes diplômées «à temps plein » de $100 \%$.

\section{Durée de recherche}

Nombre moyen de semaines, calculé à partir de la fin des études, qu'ont pris les travailleuses et les travailleurs à temps plein pour trouver un premier emploi qu'ils jugent comme un emploi d'importance.

\section{Relié à la formation}

Sont dits avoir un emploi «relié à leur formation» les travailleurs et les travailleuses à temps plein qui jugent que leur travail correspond à leurs études.

\section{Taux de chômage}

Rapport, exprimé en pourcentage, entre le nombre de personnes diplômées à la recherche d'un emploi et l'ensemble de la population active (constituée uniquement des personnes en emploi et de celles à la recherche d'un emploi).

\section{Semaine de référence}

Les données sont colligées en fonction de la semaine de référence du 19 au 25 janvier 2003 pour les titulaires d'une maitrise en 2001 et du 14 au 20 janvier 2001 pour un titulaire d'un doctorat en 1999. 



\section{Chapitre}

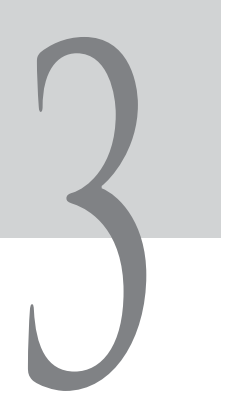

\section{LE RÔLE STRATÉGIQUE}

DES ORGANISMES SUBVENTIONNAIRES DANS LE DÉVELOPPEMENT DE LA RECHERCHE UNIVERSITAIRE ET DE LA RELÈVE SCIENTIFIQUE AU QUÉBEC

\section{Louise Dandurand}

Présidente-directrice générale,

Fonds québécois de recherche sur la société et la culture

Dans la course à l'innovation, le capital intellectuel et humain est devenu le principal actif de nos sociétés, se situant au cœur de préoccupations de nature transversale qui touchent indistinctement les entreprises, les organisations, les laboratoires et la fonction publique. Au cours des 40 dernières années, le Québec a su négocier avec succès un virage scientifique et technologique qui le situe aujourd'hui parmi les principales régions innovantes en Amérique du Nord. Dans bien des domaines, la réputation du Québec en recherche se mesure avantageusement en termes de publications scientifiques, de brevets, de participations aux grands réseaux internationaux et de rayonnement des innovations. 
Cette performance magistrale s'appuie en tout premier lieu sur un système de recherche universitaire capable à la fois de répondre à des besoins grandissants de main-d'œuvre hautement qualifiée et de développer une base de recherche solide et de nombreux créneaux d'excellence. Les prochaines années annoncent une vive concurrence, particulièrement pour attirer et former une main-d'œuvre hautement qualifiée. Il faudra donc poursuivre nos efforts pour demeurer dans la partie. Dans le contexte de la mondialisation, le maintien du Québec dans le concert des sociétés les plus avancées sera tributaire des choix que nous ferons aujourd'hui pour soutenir la recherche et l'innovation.

\section{LES GRANDS ENJEUX ET PRINCIPALES PRESSIONS CONTEXTUELLES}

En matière de soutien à la relève scientifique, il ne fait aucun doute que les trois Fonds subventionnaires du Québec ont un rôle stratégique à jouer à l'intérieur du système québécois de recherche et d'innovation. Ce rôle, ils doivent le remplir en tenant compte de deux enjeux de taille susceptibles d'avoir une influence directe sur l'insertion professionnelle des diplômés. Tout d'abord l'enjeu du rôle économique du savoir - non seulement dans la perspective restreinte d'une économie du savoir mais surtout dans la perspective élargie d'une véritable société du savoir - et, ensuite, l'enjeu du capital humain.

De ces deux principaux enjeux, qui sont sous-entendus ou en filigrane des sujets abordés dans le cadre d'un colloque comme celui-ci, découlent un certain nombre de pressions contextuelles qu'il faut prendre en considération. Pressions d'abord ressenties par la relève elle-même, mais aussi par le système de recherche et d'innovation. Pressions qui, de surcroît, interpellent les organismes subventionnaires et conditionnent leurs interventions stratégiques.

Ces pressions, on peut les regrouper en quatre catégories. Elles sont d'abord liées à l'environnement et proviennent de la dynamique démographique et de la dynamique régionale spécifiques du Québec. Elles sont ensuite lourdement influencées par la demande quantitative en main-d'œuvre hautement qualifiée (MOHQ) et par le profil qualitatif attendu de cette main-d'œuvre recherchée. 
FIGURE 3.1

Pressions contextuelles sur le système de recherche et d'innovation

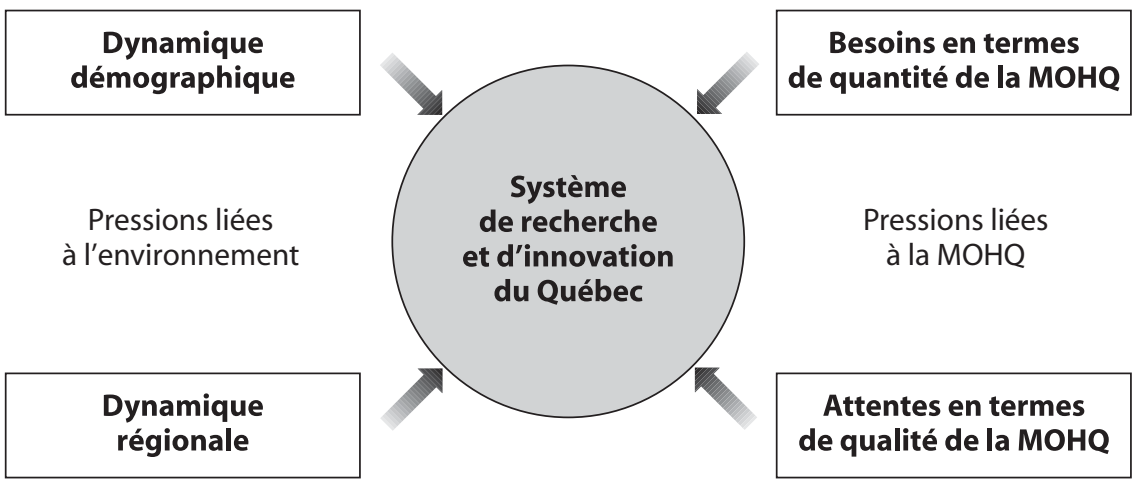

\section{Première pression : La dynamique démographique}

Au plan démographique, nous savons tous que le Québec a entamé une phase de stagnation et de déclin qui tire ses origines notamment d'un faible taux de natalité et se répercutera sous la forme d'un vieillissement de sa population. Si la tendance se maintient, le Québec comptera en 2021 davantage de personnes âgées de 65 ans et plus $(21,3 \%)$ que le Canada dans son ensemble $(17,8 \%)$ et que les États-Unis $(16,5 \%)^{1}$.

Sur le plan du marché de l'emploi, ce vieillissement se fait déjà sentir dans les secteurs privé et public ainsi que dans le milieu de l'enseignement supérieur et de la recherche. On estime ainsi que près de la moitié des besoins de main-d'œuvre des entreprises, des universités et du secteur public québécois d'ici 2006 seront causés par l'attrition découlant des départs à la retraite. L'évolution du profil démographique de la société québécoise posera de nombreux défis, notamment en matière de main-d'œuvre et particulièrement en ce qui a trait à la main-d'œuvre hautement qualifiée.

1. Le Devoir, jeudi 28 octobre 2004, page A1. 


\section{Deuxième pression : La dynamique régionale}

Les défis qui se poseront au Québec sur le plan démographique sont étroitement liés à son territoire. Dans plusieurs villes et villages, on constate déjà que le vieillissement de la population n'a pas le même impact en région quà Montréal ou Québec. L'accessibilité à des services publics de qualité et une répartition optimale de notre main-d'œuvre, surtout la $\mathrm{MOHQ}$, constituent un défi difficile à relever. Le solide réseau d'établissements collégiaux et universitaires que nous avons établi sur l'ensemble du territoire représente, à cet égard, un atout précieux.

Les hausses de clientèles constatées au cours des dernières années dans plusieurs universités situées en région montrent à quel point ces établissements jouent un rôle de premier plan non seulement dans la formation de la relève, mais aussi comme pôle d'attraction et de rétention d'une main-d'œuvre hautement qualifiée. Présentement, près de 9000 étudiants des cycles supérieurs poursuivent leur formation à TroisRivières, Rimouski, Saguenay, Gatineau, Sherbrooke et Rouyn-Noranda ${ }^{2}$. Par leur seule présence, ils contribuent au maintien de programmes de maitrise et de doctorat qui, à leur tour, soutiennent le développement d'expertises et de créneaux de recherche en lien avec les besoins et les priorités de développement des régions et permettent de rassembler des équipes de professeurs-chercheurs, soutenues par des stagiaires postdoctoraux et des professionnels de recherche. Dans l'avenir, les besoins spécifiques ressentis par les universités en région présenteront le double défi de maintenir bien vivant un patrimoine de programmes de base, entre autres au premier cycle, tout en continuant de développer des créneaux d'excellence en recherche et dans les cycles supérieurs.

\section{Troisième pression : La demande quantitative de MOHQ}

Parallèlement à l'évolution observée dans le réseau universitaire québécois, la demande de main-d'œuvre hautement qualifiée ne cesse de croître et représentera 19\% de l'ensemble de la main-d'œuvre d'ici 2006. Cet état de fait découle de la conjonction des besoins élevés de MOHQ dans les secteurs économiques en expansion, des déficits de MOHQ que l'on observe déjà dans plusieurs secteurs et des besoins accrus dans les

2. Ministère de l'Éducation du Québec, Données RECU (automne 2003), étudiants inscrits à temps complet et à temps partiel aux $2^{\mathrm{e}}$ et $3^{\mathrm{e}}$ cycles. 
domaines de recherche à fort potentiel. Bien sûr, cette demande varie selon les domaines et les secteurs. Il existe des distorsions et des cycles, et une enquête récente du Conseil de la science et de la technologie permet de bien circonscrire et de nuancer les notions de sous- et de surqualification, d'offre, de pénurie et d'équilibre du marché du travail ${ }^{3}$. Mais il n'en demeure pas moins que nous nous dirigeons assurément vers une société et un marché du travail où les besoins d'une main-d'œuvre qualifiée se feront de plus en plus sentir. Cela offre, évidemment, une chance formidable aux étudiants et aux nouveaux diplômés et représente, en même temps, tout un défi à relever.

\section{Quatrième pression : Le profil qualitatif de la MOHQ}

Au-delà des seuls besoins quantitatifs de main-d'œuvre hautement qualifiée, il nous faut nous préoccuper du profil souhaité pour cette maind'œuvre ou, en d'autres mots, de son portrait qualitatif. Nous pensons ici évidemment au niveau d'études requis et aux connaissances et compétences à développer, mais il ne faudrait pas oublier l'expérience pratique qui contribue au développement des savoir-faire et savoir-être. Ici, le défi est d'autant plus grand que les besoins sont changeants, d'un secteur d'emploi à l'autre, d'une entreprise à l'autre, en plus d'évoluer rapidement dans le temps.

\section{LA PLACE DE LA RELÈVE DANS LA MISSION DES FONDS}

Les trois Fonds de recherche du Québec accordent une très grande importance au soutien à la relève scientifique, qu'il s'agisse des étudiants de $2^{\mathrm{e}}$ et de $3^{\mathrm{e}}$ cycles, des stagiaires postdoctoraux ou chercheurs en démarrage de carrière. Il s'agit là d'un bassin considérable qui demeure au cœur des interventions concrètes des Fonds de recherche en matière de relève et des priorités dans le contexte budgétaire des dernières années.

3. Conseil de la science et de la technologie, L'avenir de la main-d'œuvre hautement qualifiée, une question d'ajustements (Avis), 2004, 235 pages. Conseil de la science et de la technologie, Les travailleurs hautement qualifiés au Québec: portrait dynamique du marché du travail, Enquête réalisée par le Centre d'étude sur l'emploi et la technologie (CETECH), 2004, 328 pages. 


\section{La relève en tant que clientèle des Fonds}

Seulement aux $2^{\mathrm{e}}$ et $3^{\mathrm{e}}$ cycles, on trouve près de 31000 étudiants à temps complet dans les universités québécoises, dont la grande majorité dans les domaines des sciences sociales et humaines, des arts et des lettres (56\%), suivis des étudiants en sciences naturelles et en génie (33\%) et des étudiants en sciences de la santé (11\%).

TABLEAU 3.1

\section{Étudiants de $2^{\mathrm{e}}$ et $3^{\mathrm{e}}$ cycle}

\begin{tabular}{|c|c|c|c|}
\hline $56 \%$ & $33 \%$ & $11 \%$ & 30958 \\
\hline SSHAL & SNG & Santé & \\
\hline
\end{tabular}

Source: MEQ, Données RECU (automne 2003), inscriptions aux cycles supérieurs à temps plein.

À la clientèle étudiante s'ajoutent les postdoctorants pour lesquels il est plus difficile d'obtenir des estimations valables. Les données recueillies par le MEQ dans le cadre de son système de comptabilisation des clientèles étudiantes (RECU) permettent néanmoins d'estimer le nombre de stagiaires postdoctorants entre 1300 et 1400 par année. Près de la moitié d'entre eux œuvrent dans le domaine des sciences naturelles et génies (SNG) (52\%), suivis du domaine de la santé (23\%) et de celui des sciences sociales et humaines, des arts et des lettres (SSHAL) (18\%). À cela s'ajoutent les postdoctorants dont l'approche ou le sujet de recherche est de nature pluridisciplinaire (7\%). Au cours des prochaines années, on pourrait même s'attendre à une augmentation du nombre de stagiaires postdoctorants. C'est du moins ce que laisse entrevoir l'augmentation de la demande de bourses postdoctorales auprès des Fonds de recherche qui a atteint cette année $73 \%$ au Fonds québécois de recherche sur la société et la culture (FQRSC), $56 \%$ au Fonds québécois de recherche sur la nature et les technologies (FQRNT) et $15 \%$ au Fonds de recherche sur la santé du Québec (FRSQ).

TABLEAU 3.2

\section{Stagiaires postdoctoraux}

\begin{tabular}{c|c|c|c|}
\hline $18 \%$ & $52 \%$ & $23 \%$ & $7 \%$ \\
\multirow{2}{*}{ SSHAL } & SNG & Santé & Autres \\
\hline
\end{tabular}

Source: MEQ, Données RECU (automne 2003), stagiaires postdoctoraux. 
En ce qui a trait aux nouveaux chercheurs, il est pour ainsi dire impossible d'évaluer correctement leur bassin, les données changeant rapidement en raison de l'intensification des embauches dans le milieu universitaire. Même à partir des données rétrospectives du Conseil supérieur de l'éducation (CSE) ${ }^{4}$, basées sur un échantillon représentatif, il est difficile d'extrapoler un bassin potentiel de nouveaux chercheurs, d'autant plus qu'on ne peut distinguer la part des postes comblés par des jeunes chercheurs de celle résultant des flux migratoires.

\subsection{Les modalités de financement de la relève}

Concrètement, la place de la relève dans les interventions des Fonds se mesure à la fois en termes de financement direct et de soutien indirect. Sur le plan du financement direct, une part importante des budgets des Fonds est consacrée à des programmes de bourses d'excellence pour étudiants de $2^{\mathrm{e}}$ et de $3^{\mathrm{e}}$ cycle, ainsi qu'à des programmes de bourses de recherche pour stagiaires postdoctoraux. On évalue ainsi que, pour une année donnée, les Fonds soutiennent directement près de 1000 étudiants de maîtrise, près de 1400 étudiants de doctorat et environ 160 stagiaires postdoctoraux, pour un total de plus de 30 millions de dollars versés en bourses.

TABLEAU 3.3

Bourses d'excellence (2002-2003) reçues par des étudiants et postdoctorants en provenance des Fonds de recherche du Québec

\begin{tabular}{|c|c|c|c|c|c|c|c|}
\hline \multicolumn{7}{|c|}{ Boursiers } & \multirow{3}{*}{$\begin{array}{l}\text { Total: } \\
2509\end{array}$} \\
\hline & \multicolumn{2}{|c|}{ Maitrise } & \multicolumn{2}{|c|}{ Doctorat } & \multicolumn{2}{|c|}{ Postdoctorat } & \\
\hline FRSQ & 171 & $2,11 \mathrm{M} \$$ & 262 & $4,02 \mathrm{M} \$$ & 31 & $0,90 \mathrm{M} \$$ & \\
\hline FQRNT & 378 & $4,04 \mathrm{M} \$$ & 475 & $4,96 \mathrm{M} \$$ & 46 & $1,02 \mathrm{M} \$$ & \multirow{3}{*}{$30,4 \mathrm{MS}$} \\
\hline \multirow[t]{2}{*}{$\mathrm{FQRSC}$} & 408 & $4,05 \mathrm{M} \$$ & 654 & $7,45 \mathrm{M} \$$ & 84 & $1,82 \mathrm{M} \$$ & \\
\hline & 957 & $10,2 \mathrm{M} \$$ & 1391 & $16,43 \mathrm{M} \$$ & 161 & $3,74 \mathrm{M} \$$ & \\
\hline
\end{tabular}

Note: Les données incluent les bourses complémentaires.

Source: Comité des PDG des Fonds de recherche du Québec, La recherche et la formation universitaires: clé de notre avenir, Mémoire présenté à la Commission parlementaire sur la qualité, l'accessibilité et le financement des universités, février 2004, page 18.

4. Conseil supérieur de l'éducation, Létat de situation et les prévisions de renouvellement $d u$ corps professoral dans les universités québécoises, 2003, 172 pages. 
De plus, des programmes ciblés de subventions offrent un soutien particulier à un groupe stratégique de la relève scientifique, celui des nouveaux professeurs-chercheurs, chercheurs-boursiers juniors et jeunes chercheurs. Un peu moins de 550 nouveaux chercheurs reçoivent ainsi, à chaque année, une subvention visant à faciliter le démarrage de leur carrière en recherche, pour un investissement total de 10,4 millions de dollars. Ainsi, les Fonds sont à même de soutenir les éléments les plus prometteurs de la relève scientifique du Québec, à partir du $2^{\mathrm{e}}$ cycle universitaire jusqu'au démarrage de leur carrière universitaire en recherche, en passant par le doctorat et le postdoctorat.

TABLEAU 3.4

\section{Programmes de subventions ciblés pour le soutien au démarrage de carrière en recherche (2003-2004)}

\begin{tabular}{|c|c|c|c|}
\hline \multicolumn{3}{|c|}{ Programmes de soutien à la relève de chercheurs } & \multirow{3}{*}{$\begin{array}{c}\text { Total: } \\
547\end{array}$} \\
\hline FRSQ & 100 & $1,5 \mathrm{M} \$$ & \\
\hline FQRNT & 173 & $3,8 \mathrm{M} \$$ & \\
\hline \multirow[t]{2}{*}{ FQRSC } & 274 & $5,1 \mathrm{M} \$$ & \multirow[t]{2}{*}{$10,4 \mathrm{MS}$} \\
\hline & 547 & $10,4 \mathrm{M} \$$ & \\
\hline
\end{tabular}

Source: Rapports annuels 2003-2004 FRSQ, FQRNT et FQRSC.

Tout aussi important sur le plan des investissements, le soutien indirect à même les subventions versées aux chercheurs individuels et regroupés au sein d'équipes, de centres ou de laboratoires de recherche, permet à plusieurs centaines d'étudiants et de stagiaires postdoctoraux de bénéficier de contrats. Il s'agit d'une occasion de parfaire leurs connaissances et d'approfondir leur formation pratique en recherche, en plus de représenter, pour plusieurs d'entre eux, une source de revenu importante dans la poursuite de leurs études.

On évalue ainsi entre 3200 et 3800 le nombre d'étudiants de tous les cycles et stagiaires postdoctoraux bénéficiant d'un contrat de recherche financé à même les subventions versées par le Fonds Nature et Technologies et par le Fonds Société et Culture (aucune donnée comparable n'est disponible pour le FRSQ). On remarquera que le soutien indirect est substantiel: tout près de 18 millions de dollars en excluant la part du FRSQ. C'est 33\% du budget des subventions du FQRNT et $45 \%$ de celui du FQRSC qui vont au soutien à la relève (assistanats et contrats de 
recherche). Et cela, sans compter les contrats d'embauche de professionnels de recherche qui constituent, pour plusieurs jeunes diplômés, un premier pas sur le marché du travail dans le domaine de la recherche.

TABLEAU 3.5

Parts des subventions de recherche (2001-2002) reçues par des étudiants et postdoctorants en provenance des Fonds de recherche du Québec

\begin{tabular}{|c|c|c|c|}
\hline \multicolumn{3}{|c|}{ Sous contrat } & \multirow{6}{*}{$\begin{array}{c}\text { Total: Entre } \\
3200 \text { et } 3800 \\
\text { 17,9 M \$ (et plus) }\end{array}$} \\
\hline & \multicolumn{2}{|c|}{ Tous les cycles } & \\
\hline FRSQ & N.D. & N.D. & \\
\hline FQRNT & 1627 & $7,7 \mathrm{M} \$$ & \\
\hline \multirow[t]{2}{*}{ FQRSC } & 1612 & $10,2 \mathrm{M} \$$ & \\
\hline & 3236 & $17,9 \mathrm{M} \$$ & \\
\hline
\end{tabular}

Note: Les données incluent les $1^{\mathrm{er}}, 2^{\mathrm{e}}, 3^{\mathrm{e}}$ cycles et les postdoctorants. À noter que, dans le cas du FQRSC, il manque des informations concernant le nombre d'étudiants des anciens programmes du CQRS. En conséquence, on peut estimer à environ 2150 le nombre d'étudiants et postdoctorants sous contrat (plutôt que 1612). Par contre, aucune estimation n'est disponible pour ce qui est des montants investis.

Source: Comité des PDG des Fonds de recherche du Québec, La recherche et la formation universitaires : clé de notre avenir, Mémoire présenté à la Commission parlementaire sur la qualité, l'accessibilité et le financement des universités, février 2004, page 18.

FIGURE 3.2

\section{Budget des subventions au FQRNT et au FQRSC}

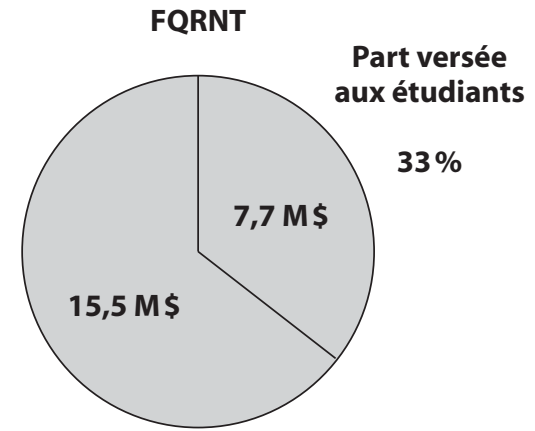

Budget total des subventions: 23,2 M\$

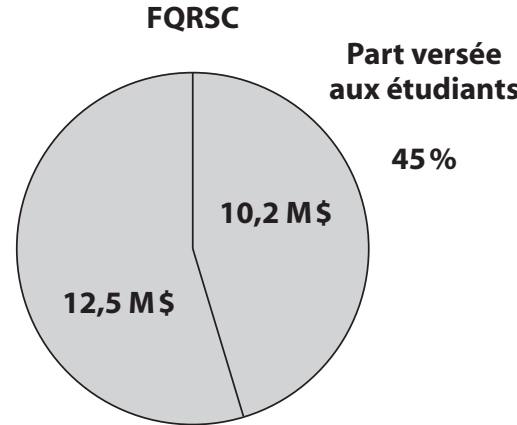

Budget total des subventions:

22,7 M\$ 
En 2002-2003, le soutien direct et indirect à la relève pour les trois Fonds a donc totalisé 58,7 millions de dollars; cela ne comprend pas le soutien indirect du FRSQ qui comptabilise différemment les postes budgétaires. En soutien direct, la plus grosse part va aux étudiants de doctorats, suivis des nouveaux chercheurs, des étudiants de maîtrise et des postdoctorants. On comprendra aisément que, sans une augmentation du portefeuille de bourses, on peut difficilement faire plus. Et c'est malheureux, puisque cette année par exemple, pour honorer l'ensemble des recommandations des comités d'évaluation des demandes de bourses de $2^{\mathrm{e}}$ et $3^{\mathrm{e}}$ cycle, 43 millions de dollars supplémentaires auraient été nécessaires, ce qui représenterait une augmentation de $30 \%$ du budget global des trois Fonds! Un soutien adéquat, en termes de taux, de hauteur et de durée de financement est irréalisable dans le contexte actuel, même s'il demeure très certainement souhaitable dans la perspective d'un réinvestissement en recherche.

\section{Les mesures de soutien à la relève}

Au-delà des modalités de soutien financier, les Fonds ont également mis en place un ensemble de mesures ciblant la relève. L'importance qu'ils accordent au soutien à la relève se concrétise notamment dans l'évaluation des conditions d'encadrement et de formation qui sont des critères formels d'évaluation des demandes de subvention. Les Fonds ont également adopté des mesures spécifiques visant, entre autres, à faciliter la conciliation famille-étude. Ces mesures prévoient un versement supplémentaire et permettent l'allongement de la fenêtre d'admissibilité afin de mieux soutenir les jeunes parents boursiers. Les Fonds développent aussi présentement un projet de suivi des boursiers qui inclura un rapport final et une relance auprès des boursiers. Le suivi permettra notamment de documenter la durée des études, l'insertion professionnelle et la mobilité géographique en fonction du profil de formation et du soutien financier. Enfin, du côté des stagiaires postdoctoraux, un chantier est en cours afin d'améliorer le statut et la reconnaissance des postdoctorants, dans la foulée du dépôt du rapport Milot ${ }^{5}$.

5. P. Milot, La formation postdoctorale financée par les conseils subventionnaires, Rapport du CIRST, 2003, 77 pages. 
La liste de ces mesures pourrait évidemment s'allonger dans l'éventualité d'un réinvestissement en recherche: on pense par exemple à des incitatifs à la recherche au premier cycle, à des bourses dans les domaines stratégiques ou en émergence, à l'internationalisation de la formation, au retour des étudiants québécois de l'étranger... Clairement, ce ne sont ni les idées, ni la volonté qui manquent.

\section{Quelques remarques sur des propositions récentes de la FEUQ}

D'ailleurs, la «cause» d'un réinvestissement en recherche a été très bien servie lors de la Commission parlementaire sur l'accessibilité, la qualité et le financement des universités, notamment par le Conseil national des cycles supérieurs (CNCS) de la Fédération étudiante universitaire du Québec (FEUQ). Sur les 52 recommandations du mémoire du CNCS, 22 touchaient la recherche et 13 visaient directement les Fonds de recherche. Quatre d'entre elles concernaient le soutien financier aux étudiants des cycles supérieurs. Dans le cadre d'un colloque comme celui-ci, il convient d'analyser ces recommandations et d'en nuancer les implications du point de vue du Fonds Société et Culture que je préside. Deux principes de décision qui guident les choix stratégiques du Fonds dans le contexte d'un budget constant, soit l'équité intercohorte et les vases communicants, doivent être clarifiés ici afin de mieux comprendre l'impact potentiel de ces recommandations.

La recommandation $\mathrm{n}^{\circ} 34$ du mémoire du CNCS propose d'augmenter la période d'admissibilité aux bourses. Cela pourrait se réaliser par l'allongement de la durée du financement ou par l'élargissement de la fenêtre d'admissibilité. Malheureusement, dans les deux cas, on pourrait craindre un problème d'équité intercohorte. En effet, à budget constant, prolonger la période de financement d'une cohorte donnée se ferait au détriment des cohortes suivantes puisque la hauteur des engagements pluriannuels d'une année donnée diminuerait d'autant les sommes disponibles pour les concours des années subséquentes. D’un autre côté, allonger la période d'admissibilité poserait un problème similaire, puisque des étudiants débutant leur programme se trouveraient en concurrence avec des étudiants très avancés dans leur projet de recherche. Déjà, avec la période maximale et le nombre de demandes permises qui prévalent présentement, on constate dans certains cas l'existence d'une telle distorsion intercohorte. 
Trois autres recommandations ( $\mathrm{n}^{\mathrm{os}} 42,43$ et 44$)$ portaient quant à elles sur le soutien indirect aux étudiants. Le CNCS souhaiterait que l'on attribue une part croissante du soutien indirect aux étudiants et que l'on établisse un seuil minimal de $25 \%$ qui serait réservé aux étudiants dans chacune des subventions octroyées. Dans les données présentées précédemment, on a pu constater que ce minimum est déjà largement atteint globalement. L'imposition d'une proportion minimale systématique a déjà été tentée, puis retirée des Règles générales communes des Fonds, et cela, dans une visée de souplesse et parce que dans certaines pratiques de recherche et certains contextes institutionnels l'embauche de professionnels est un impératif. Par ailleurs, il ne faudrait pas oublier que ces embauches représentent des opportunités d'insertion professionnelle pour les nouveaux diplômés. Même s'il s'agit d'emplois sous octroi de recherche qui sont, par définition, de nature plus précaire, ce sont néanmoins des emplois de choix, en ce sens qu'ils permettent aux nouveaux diplômés de commencer leur carrière en recherche avec les meilleurs chercheurs et au sein d'équipes, de centres et de groupes de recherche de premier plan. Vous voyez bien donc qu'à budget constant, augmenter le portefeuille des bourses, c'est diminuer celui des subventions et donc sabrer dans le soutien indirect aux étudiants... et vice-versa, puisqu'il s'agit de vases communicants. Un cercle vicieux en quelque sorte dont les étudiants seraient les premières victimes.

\section{EN CONCLUSION}

Il est clair que le système de recherche et d'innovation a un rôle à jouer en ce qui a trait à la préparation d'une relève scientifique qui réponde aux attentes et permette de combler les besoins de main-d'œuvre hautement qualifiée sentis tant dans le secteur privé, le secteur gouvernemental et le milieu de l'enseignement supérieur et de la recherche. Et cela, non seulement sur le plan du financement, mais aussi comme partenaire du milieu de l'enseignement supérieur et de la recherche. Que ce soit sur le plan de l'éveil et de la culture scientifiques, sur celui de l'esprit et de la pratique scientifique, de l'évolution des contenus d'enseignement et des programmes de formation, de la valorisation des carrières scientifiques dans les secteurs privés et publics et de l'attrait du «marché» universitaire... la capacité du Québec à disposer d'une main-d'œuvre hautement qualifiée, dans un contexte où la compétition ne connaît plus de frontière, sera le principal enjeu auquel nous serons confrontés. 
Nous n'avons guère le choix que d'outiller nos universités et de les doter d'un environnement stimulant et d'une forte capacité de recherche. Des conditions optimales de financement et d'encadrement de la recherche permettront de mieux arrimer la formation aux besoins du marché, de soutenir le démarrage de carrières de recherche et de favoriser la consolidation d'entités et d'expertises.

Voilà à la fois les défis qui nous attendent comme société... et les moyens de poursuivre notre développement scientifique, social, économique, technologique et culturel. Voilà aussi le cœur même de la mission des Fonds de recherche du Québec. 



\section{Chapitre}

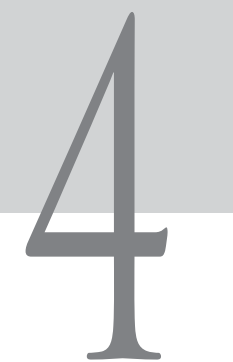

\section{RENFORCER L'ACQUISITION DES COMPÉTENCES ET LA GESTION DES CARRIÈRES}

\section{Sylvie Dillard}

Présidente-directrice générale,

Fonds québécois de recherche sur la nature

et les technologies

Dans son dernier avis, L'avenir de la main-d'ouvre hautement qualifiée (février 2004), le Conseil de la science et de la technologie apporte des éléments de diagnostic très importants et nouveaux, qui questionnent la tendance généralisée à considérer les problèmes de personnel hautement qualifié strictement en termes quantitatifs et en mettant l'accent sur l'insuffisance de l'offre.

Or les analyses du CST démontrent qu'il n'y a pas de pénuries généralisées, mais des problèmes d'ajustements quantitatifs et qualitatifs entre l'offre et la demande de personnel scientifique et technique sur un marché du travail qui se resserre.

Également, il n'y aurait pas de désaffection des jeunes à l'égard des sciences, mais une certaine redistribution des inscriptions entre les sciences pures ou naturelles et les sciences de la santé. Le niveau relativement élevé des taux de chômage des jeunes diplômés en sciences 
naturelles préoccupe le Conseil, qui conclut à la nécessité de stimuler la demande, avant d'envisager une production massive de nouveaux diplômés dans ces domaines.

Ce type de préoccupations commence d'ailleurs à se répandre largement, aux États-Unis comme en Europe (voir Michel Teitelbaum, Do We Need More Scientists? 2003).

\section{LES TROIS GRANDS DÉFIS DU QUÉBEC}

Le capital humain très qualifié est l'enjeu majeur $d u X X I^{e}$ siècle; il est le nerf de la guerre dans la course à l'innovation. Cependant, le Québec n'est pas nécessairement en position de force.

Le Québec a en effet trois grands défis à relever: maintenir l'offre, stimuler la demande, améliorer l'adéquation entre l'offre et la demande de personnel très qualifié pour l'entreprise.

\section{Du côté de l'offre}

\section{Le Québec se trouve dans une situation critique en ce qui a trait au bassin des vocations pour les raisons suivantes:}

- Un vieillissement démographique plus rapide qu'ailleurs : la proportion des personnes âgées de 65 ans et plus passera, au Québec, de 12 à $27 \%$ d'ici 2030. Ce n'est qu'en 2045 que le reste du Canada atteindra une telle proportion (en 2060 aux États-Unis; Conseil de la science et de la technologie, 2004);

- Les femmes démontrent un intérêt moindre pour les sciences naturelles, en particulier le génie, alors qu'elles sont les principales responsables de l'accroissement des inscriptions universitaires : en sciences pures et appliquées, les diplômées sont toujours moins nombreuses à la maîtrise (36\%) et au doctorat (26\%) que leurs pairs masculins;

- Les hommes sont traditionnellement plus intéressés à ce type de carrière, mais réussissent moins bien que les femmes tout au long du parcours universitaire;

- La proportion d'étudiants étrangers est très élevée dans de nombreux laboratoires de recherche en génie; au doctorat, près de $24 \%$ des étudiants en sciences pures et $32 \%$ en sciences appliquées 
ne sont pas citoyens canadiens, alors que la proportion est de $20 \%$ pour l'ensemble des disciplines. Mais ce n'est qu'une faible proportion d'entre eux qui restera au Québec (MEQ, compilation spéciale).

\section{Le Québec est soumis à une vive concurrence pour ce qui est de l'attrait et de la rétention du capital humain très qualifié.}

- Le marché de la recherche se globalise et s'agrandit.

- La surenchère pour le personnel qualifié s'accroît, en particulier dans les pays développés qui mettent au point des dispositifs ingénieux pour attirer les «travailleurs stratégiques».

- Le renouvellement du corps professoral dans toutes les universités canadiennes crée une pression importante, particulièrement dans certains secteurs, tels que le génie ou l'informatique.

- La pression du marché du travail est forte, faisant en sorte que dans certains secteurs, les candidats sont embauchés avant même d'avoir terminé leur scolarité. Le marché du travail est particulièrement attractif pour les diplômés de premier cycle en sciences appliquées: seulement $12 \%$ poursuivent des études supérieures, alors que la proportion est de $20 \%$ pour l'ensemble des finissants (MEQ, 2001).

\section{Alors que les débouchés se trouvent aujourd'hui majoritairement hors du milieu universitaire, la formation n'a pas suivi.}

- Près des deux tiers des titulaires d'un doctorat en SNG travaillent dans un milieu autre que l'université.

- Les employeurs se plaignent d'un manque de compétences personnelles et professionnelles (autoreproduction du système universitaire), d'un manque d'expérience et d'ouverture aux milieux non institutionnels, d'un manque de largeur en $\mathrm{S}$ et $\mathrm{T}$ : "Nous ne voulons pas qu'il creuse dans le même trou, mais qu'il sache où creuser» (Nyquist, 2002).

- Les étudiants se plaignent d'une qualité d'encadrement inégale et insuffisante, ainsi que d'un manque d'informations et de préparation adéquate aux différentes carrières, en particulier dans l'entreprise privée. 
- Cette situation est particulièrement flagrante pour la formation doctorale: peu de valorisation du doctorat des milieux universitaires: «trop rigide, trop pointu, trop tard» (Forum de l'Association de la recherche industrielle du Québec [ADRIQ] sur les partenariats université-entreprise, juin 2004).

- De nombreuses initiatives sont en cours au plan international depuis cinq à huit ans pour repenser cette formation: Integrative Graduate Education and Research Training de la NSF (É-U.), Teaching Company Scheme (Royaume-Uni), Écoles doctorales en France et en Allemagne.

- Au Québec et au Canada, plusieurs approuvent la mise sur pied d'une formation aux cycles supérieurs qui permet de mieux arrimer les formations aux exigences et aux pratiques de la R-D en milieu industriel (Ateliers industriels du Conseil de recherches en sciences naturelles et génie du Canada [CRSNG], mai 2003; Mémoire de l'ADRIQ dans le cadre des consultations sur la stratégie d'innovation du Canada en 2002).

\section{Du côté de la demande}

\section{Le personnel très spécialisé en entreprise constitue un maillon très faible, ce qui freine l'innovation dans l'entreprise, en particulier la PME.}

- Le poids des chercheurs en entreprise par 10000 de population active (41) est sensiblement inférieur à celui de l'Ontario (45) et de la moyenne des pays du G7 (51). (Ministère du Développement économique et régional et de la Recherche, 2003.)

- Parmi le personnel affecté à la R-D, le poids des professionnels affectés à la R-D (60\%) est largement inférieur à la moyenne nationale (66\%) et à l'Ontario (71\%). (Statistique Canada, 2002.)

- Seulement $6 \%$ du personnel professionnel affecté à la R-D dans les entreprises québécoises est titulaire d'un doctorat, comparé à 6,6\% pour la moyenne canadienne; cette proportion est en diminution depuis cinq ans. (Statistique Canada, 2003).

- De 1996 à 2001, le Québec, qui décerne 25\% des doctorats, n'en récolte que $15 \%$ dans la population active. (Statistique Canada 2004, compilation spéciale). 
- Selon le Centre de recherche industrielle du Québec (CRIQ), il existe un écart préoccupant entre le niveau de productivité des entreprises manufacturières du Québec et celui des mêmes entreprises de l'Ontario (23\%) et des États-Unis (35\%). Or les entreprises qui affichent un niveau de productivité élevé s'appuient sur leur capital humain pour développer des stratégies d'innovation faisant appel aux qualifications des ressources humaines telles que l'ingénierie, la conception industrielle et la formation (Institut de la statistique du Québec, 2002).

- Les PME innovantes dans le secteur de la fabrication emploient plus d'employés titulaires d'un diplôme en SNG (78\%) que les autres $(50 \%)$ : «le faible recours à l'expertise externe par les entreprises non innovantes peut être causé par le manque de ressources internes susceptibles d'intégrer les connaissances» (Institut de la statistique eu Québec, 2002).

La concurrence des économies émergentes (Inde, Chine) pour la production technologique courante milite en faveur d'une spécialisation accrue des entreprises québécoises vers les produits spécialisés et le rehaussement de la qualification de la main-d'œuvre.

- «En fait, nous sommes coincés dans une position intermédiaire limitée, des pays comme la Chine nous dépassant du point de vue de la production technologique courante, d'une part, et les ÉtatsUnis nous dépassant du point de vue des technologies de pointe, d'autre part.» (Industrie Canada, 2004)

Le développement économique des régions passe par un rehaussement de leurs compétences en innovation, dans un contexte de concurrence difficile avec les grands centres urbains, pour ce qui est de l'attraction et de la rétention d'entreprises et de personnel très qualifié.

- Pour chaque millier de personnes actives, le personnel de R-D en entreprise atteint 24,2 à Montréal, par opposition à 4,5 pour l'ensemble des autres régions (Tableau de bord des systèmes régionaux d'innovation du Québec, 2004). 


\section{L'enjeu " qualitatif " d'adaptation entre l'offre et la demande de personnel très qualifié peut se résumer dans le tableau suivant :}

TABLEAU 4.1

Recherche universitaire et R-D-I (Recherche - Développement Innovation) en entreprise : une nature et des objectifs différents

\begin{tabular}{|l|l|}
\hline Recherche universitaire & Recherche en entreprise \\
\hline Conditions non contrôlées & Conditions contrôlées \\
Création de la valeur intellectuelle & Réalisation de la valeur économique \\
Crédibilité scientifique & Applicabilité industrielle \\
Axée sur une mission & Axée sur le marché \\
Déductive ou analytique & Inductive ou synthétique \\
Axée sur les disciplines & Axée sur les problèmes (transdisciplinaire) \\
Découvertes fortuites / curiosité & Accomplissement (progression vers un but) \\
Pas de contraintes de temps & Calendriers à respecter \\
Biens publics & Biens privés (propriété) \\
\hline «Publish or perish» & «Innovate or evaporate» (Higgins) \\
\hline
\end{tabular}

Source: Tumiura, 2002, cité dans Jean Nicolas, Rapport du chantier sur la Relève (2002).

\section{DES PISTES D'ACTION POUR L'AVENIR}

Il apparaît urgent pour le Fonds Nature et Technologies, comme pour un bon nombre de partenaires consultés, employeurs et employés potentiels, que le Québec prenne exemple sur d'autres pays et mette en place des initiatives novatrices pour améliorer à la fois l'employabilité de nos jeunes diplômés des cycles supérieurs et les compétences en innovation des entreprises et des régions.

Deux types de démarches ont été entreprises par le Fonds Nature et Technologies:

1. Pour améliorer nos connaissances sur les carrières de nos diplômés des cycles supérieurs, particulièrement au doctorat:

- On a veillé à l'implantation dès janvier 2005 d'un système de suivi des boursiers pour les trois Fonds de recherche du Québec;

- On a entrepris démarches répétées auprès du ministère de l'Éducation (MEQ), visant la récurrence de l'enquête Relance du MEQ auprès des titulaires d'un doctorat. 
2. Pour favoriser l'accroissement de l'employabilité de nos jeunes diplômés des cycles supérieurs, ainsi que les compétences en innovation des entreprises

- Il est sérieusement envisagé de relancer le programme de Bourses en milieu de pratique (BMP), qui a reçu une évaluation très positive tant de la part des étudiants, des directeurs de recherche universitaires, que des superviseurs en milieu de pratique. Des démarches sont en cours à cet effet auprès du MDERR et de nombreux partenaires, incluant le CRSNG, afin d'obtenir le financement nécessaire. 



\title{
Chapitre
}

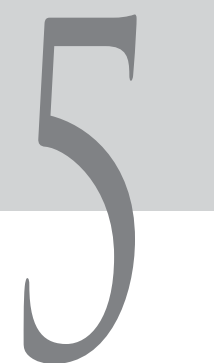

\section{LE RENOUVELLEMENT ET LE DÉVELOPPEMENT DU CORPS PROFESSORAL UNIVERSITAIRE}

\section{L'identification des besoins des universités québécoises ${ }^{1}$}

\author{
Jean-Pierre Proulx \\ Président, \\ Conseil supérieur de l'éducation
}

Quelles sont les prévisions d'embauche des professeurs dans les universités québécoises d'ici 2008 ? C'est la question toute simple à laquelle a voulu répondre le Conseil supérieur de l'éducation dans son rapport annuel 2002-2003 tout entier consacré au renouvellement du corps professoral $^{2}$. Ce n'était évidemment pas une question de curiosité. Le

1. Je suis entièrement redevable pour ce texte à Mme France Picard, coordonnatrice de la Commission de la recherche et de l'enseignement universitaires du Conseil supérieur de l'éducation, qui a coordonné les études menant à l'élaboration du rapport annuel sur le renouvellement du corps professoral. Je lui sais gré de m'avoir permis d'utiliser les notes dont elle s'est servie lors de la conférence qu'elle a prononcée, le 12 février 2004, au colloque Le renouvellement $d u$ corps professoral, organisé par l'Association francophone pour le savoir - ACFAS, le Conseil supérieur de l'éducation, la Fédération québécoise des professeures et professeurs d'université (FQPPU), le Conseil national des cycles supérieurs (CNCS) et la Conférence des recteurs et des principaux des universités du Québec (CREPUQ). 
renouvellement du corps professoral universitaire constitue un enjeu fondamental pour l'accomplissement même de la mission des universités.

Pour répondre à la question, le Conseil a mené une enquête auprès des vice-recteurs aux ressources humaines des universités québécoises ${ }^{3}$. La présente communication présente quelques résultats issus de cette enquête.

$\mathrm{Au}$ regard des prévisions de recrutement établies par le Conseil pour les universités québécoises, on rend compte des hypothèses à la base des prévisions établies dans chaque établissement. On pourra ainsi mieux comprendre les écarts entre le scénario prévisionnel du Conseil, celui de l'Association des universités et collèges du Canada (AUCC) et celui publié par la CREPUQ au début de l'année 2004.

Après avoir expliqué brièvement les aspects méthodologiques, on rendra compte des prévisions recueillies dans les universités québécoises pour la période 2003-2004 à 2007-2008 tout en approfondissant les hypothèses sur lesquelles ces prévisions sont fondées.

\section{LA MÉTHODOLOGIE}

Le but de l'étude était d'établir des prévisions de recrutement des professeurs qui mettent l'accent sur les universités québécoises et qui permettent de cerner ce recrutement selon les domaines d'enseignement et de recherche ainsi que selon les caractéristiques des établissements.

Les variables prises en compte découlent de ce but. On a contrôlé le domaine d'enseignement et de recherche, en utilisant la typologie CLARDER et la taille du corps professoral des universités. On a aussi pris en compte la langue d'enseignement et la situation géographique.

2. Conseil supérieur de l'éducation, Renouveler le corps professoral à l'université: des défis importants à mieux cerner. Rapport annuel sur l'état et les besoins de l'éducation 2002-2003, Québec, Le Conseil, 2003. Le rapport est disponible sur le site du Conseil à l'adresse suivante: <www. cse.gouv.qc.ca>. Cette remarque s'applique également à l'étude citée à la note suivante.

3. Jean-François Lehoux, France Picard et Gilles Roy, Létat de la situation et les prévisions de renouvellement du corps professoral dans les universités québécoises. Rapport de deux enquêtes effectuées auprès des administrateurs universitaires, Québec, Conseil supérieur de l'éducation, janvier 2004. Ce rapport traite aussi d'une première enquête menée auprès des directeurs de département sur la situation du recrutement des professeurs entre janvier 1997 et décembre 2002. 
On a ainsi pu recueillir des données inédites sur les prévisions de recrutement au Québec. On a pu montrer l'évolution prévisible du corps professoral selon les domaines et les caractéristiques des établissements.

Les limites de l'étude tiennent à la nature même de son objet. En effet, une prévision, c'est essentiellement une approximation de ce qu'il adviendra dans le futur à partir d'un ensemble d'hypothèses formulées. En ce qui touche les prévisions de recrutement des professeurs d'universités, elles se fondent sur des hypothèses qui concernent:

- L'ampleur des départs (combien prendront leur retraite et à quel âge, l'importance de la mobilité des professeurs en cours de carrière, la capacité d'un établissement à garder ses professeurs en poste) ;

- La décision de remplacer ou non ces départs;

- Les orientations de développement selon les domaines;

- La fréquentation universitaire;

- Le financement nécessaire à l'embauche des professeurs.

Dans le rapport annuel (voir la section 1.3), on a formulé un modèle de renouvellement du corps professoral qui insiste sur la diversité des facteurs en jeu, sur l'équilibre dynamique de ces facteurs et, de ce fait, sur l'incertitude entourant les prévisions d'embauche. Les facteurs de conjoncture peuvent faire varier considérablement les prévisions établies.

Des contacts ont été établis avec le personnel du siège social de la CREPUQ ainsi qu'avec le vice-rectorat aux ressources humaines dans un établissement pour valider certains aspects de la démarche de collecte de données, dont le groupe d'interlocuteurs le plus apte à établir de telles prévisions, la faisabilité de la démarche, la période visée par les prévisions ( 5 ans au lieu de 10 ans comme prévu), la clarté des variables («professeurs réguliers») et l'utilisation de la typologie CLARDER.

\section{LA COLLECTE DE DONNÉES}

On a choisi de confier aux établissements le soin de faire leurs propres prévisions. Un formulaire précisait les informations demandées. En outre, il était demandé de décrire de manière explicite les hypothèses à partir 
desquelles étaient établies les prévisions. Le formulaire a été envoyé par la poste aux vice-recteurs aux ressources humaines ou, dans certains cas, aux «doyens» ou aux directeurs des ressources humaines.

Tous les établissements ont complété les prévisions demandées pour leur établissement. Aucune hypothèse n'a été posée a priori par le Conseil.

\section{LES PRÉVISIONS 2003-20084}

Voici le tableau des prévisions de recrutement des professeurs recueillies par le Conseil. Selon le scénario prévisionnel établi sur la base des données fournies par les vice-recteurs aux ressources humaines, les universités québécoises comptent recruter de 2003-2004 à 2007-2008:

- 3071 professeurs,

- dont 2025 pour combler les départs,

- et 1046 postes additionnels,

- l'effort total de recrutement estimé à 37,3\% du corps professoral de l'automne 2001.

Comment expliquer un tel niveau de recrutement?

De fait, la situation dans les universités est très variable et les niveaux de recrutement le sont par conséquent. L'effort de recrutement prévu varie entre $16,7 \%$ et $95 \%$ du corps professoral de 2001 d'un établissement pour la période mentionnée. Il y a donc des écarts importants dans les prévisions des établissements. C'est d'ailleurs ce que montre le tableau 5.1.

TABLEAU 5.1

\section{Variation de l'effort de recrutement prévu selon les universités}

\begin{tabular}{|l|c|}
\hline $\begin{array}{l}\text { Pourcentage du recrutement } \\
\text { sur le corps professoral } 2001\end{array}$ & Nombre d'universités \\
\hline - entre $75 \%$ et $95 \%$ & 3 \\
- entre $55 \%$ et $75 \%$ & 2 \\
- entre $35 \%$ et $55 \%$ & 6 \\
\hline - entre $15 \%$ et $35 \%$ & 7 \\
\hline
\end{tabular}

4. On se référera au tableau 55, p. 85, et aux tableaux 56 et 57, p. 87, de létude citée à la note 3 . 
Voici les hypothèses retenues pour expliquer les prévisions. Douze établissements sur dix-huit ont apporté les précisions demandées à cette fin.

Si l'on considère le premier groupe d'universités qui ont au moins l'équivalent de l'effort total de recrutement estimé $37,3 \%$, c'est-à-dire les données au-dessus de la ligne blanche, les hypothèses que les vice-recteurs aux ressources humaines retiennent pour soutenir leurs prévisions de recrutement sont les suivantes:

- On prévoit remplacer tous les départs.

- On projette de créer de nouveaux postes afin:

- de développer certains créneaux de formation et de recherche;

- d'améliorer le ratio professeur-étudiants;

- de prendre en compte l'augmentation des étudiants de cycles supérieurs;

- de transformer des postes temporaires en postes réguliers;

- de tenir compte d'une augmentation prévue de la population étudiante.

- On fait l'hypothèse d'un financement public additionnel.

- On tient compte du fait que le recrutement de jeunes professeurs entraîne une mobilité professionnelle plus grande.

Néanmoins, même dans ces cas d'efforts intenses de recrutement:

- On prévoit développer des créneaux de formation et de recherche en permutant des postes entre les domaines, à la suite de départs.

- On formule certaines hypothèses sur la restriction prévue du nombre de départs. Ainsi, on prévoit que les départs devraient diminuer compte tenu d'un rajeunissement progressif du corps professoral, que les départs à la retraite devraient se restreindre, comparativement à ce qui a été observé vers la fin des années 1990 et on ne prévoit pas de départ à la retraite avec pénalité.

- On prévoit également mettre en œuvre des mesures particulières pour restreindre l'attrition et favoriser l'intégration des nouveaux professeurs. 
Examinons maintenant les hypothèses formulées par la dernière catégorie d'établissements dont l'effort de recrutement devrait se situer entre $15 \%$ et moins de $35 \%$ de leur corps professoral d'octobre 2001 .

- Tous les départs seront comblés.

- Il n'y aura aucune création de postes, malgré une légère augmentation de la population étudiante.

- Dans le cas de création de postes, elle se restreint à un niveau très faible.

- On procédera au développement de créneaux de formation et de recherche en permutant des postes d'un domaine à l'autre à la suite de départs.

- On fait l'hypothèse que le financement public n'augmentera pas.

Ces hypothèses et ce tableau sur la distribution de fréquence en fonction de l'effort de recrutement prévu expliquent le résultat obtenu par le Conseil dans son scénario prévisionnel.

\section{LES PRÉVISIONS SELON LES CARACTÉRISTIQUES DES UNIVERSITÉS}

Un deuxième tableau ventile les prévisions globales selon les caractéristiques des établissements. Ces données permettent en outre d'écarter certaines interprétations erronées qui ont été véhiculées à partir des données.

TABLEAU 5.2

\section{Les prévisions selon les caractéristiques des universités}

\begin{tabular}{|l|c|c|}
\hline & Recrutement prévu & $\begin{array}{c}\text { Pourcentage sur le corps } \\
\text { professoral 2001 }\end{array}$ \\
\hline Total & 3071 & $37,3 \%$ \\
\hline 500 professeurs ou plus & 2482 & $36,1 \%$ \\
\hline 500 professeurs ou moins & 589 & $43,3 \%$ \\
\hline Centres urbains & 2727 & $37,8 \%$ \\
\hline Régions & 344 & $33,7 \%$ \\
\hline Français & 2181 & $36,1 \%$ \\
\hline Anglais & 890 & $40,6 \%$ \\
\hline
\end{tabular}


Ainsi, au regard du recrutement prévu de 3071 professeurs, qui représente un effort de $37,3 \%$ du corps professoral d'octobre 2001 :

- On observe que c'est dans les universités de moins de 500 professeurs que l'effort de recrutement prévu devrait être le plus intense. Il est vrai de dire que cette dernière catégorie regroupe l'ensemble des universités en région, mais la donnée suivante montre que ce niveau n'est pas attribuable à cette catégorie d'établissements.

- On note ainsi que pour les universités des centres urbains - Québec, Montréal et Sherbrooke - l'effort est le plus intense, soit 37,8\% par rapport à 33,7\% en région. Selon Emploi-Québec, on peut également qualifier ce dernier taux d'élevé ${ }^{\text {. }}$

- Finalement, on constate que cet effort de recrutement, selon les prévisions, devrait être plus grand dans les établissements de langue anglaise que ceux de langue française.

5. Selon Emploi-Québec, un remplacement de main-d'œuvre de plus de $23,7 \%$ sur 5 ans est élevé. 



\section{Chapitre}

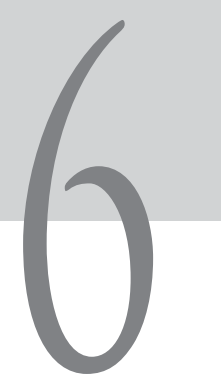

\section{FORMATION EN MILIEU PRATIQUE ET INSERTION PROFESSIONNELLE DES JEUNES CHERCHEURS}

\section{Brigitte Gemme}

Centre interuniversitaire de recherche

sur la science et la technologie (CIRST)

\section{Yves Gingras}

Chaire de recherche du Canada en histoire

et sociologie des sciences

Les pratiques de recherche des universitaires se transforment et s'ouvrent davantage aux préoccupations et aux besoins de leur milieu social, politique et économique, notamment sous l'effet des nouveaux modes de financement de la recherche (Godin, Trépanier et Albert, 2002; Slaughter et Leslie, 1997). Ces transformations ont un impact non seulement sur le travail et la carrière des professeurs-chercheurs, mais également sur l'expérience de formation des étudiantes et étudiants qui, sous leur direction, apprennent à faire de la recherche (Gemme et Gingras, à paraître). En introduisant de nouvelles manières de percevoir et de faire dans l'habitus scientifique traditionnellement acquis par les étudiants aux cycles supérieurs, ces nouvelles pratiques auront-elles aussi des impacts sur l'insertion professionnelle des diplômés? À l'aide des données recueillies auprès de 956 d'étudiants et diplômés des programmes de maîtrise et 
de doctorat ${ }^{1}$ québécois, nous examinerons les perspectives d'insertion professionnelle et les trajectoires effectives associées aux divers types de formation. Plus spécifiquement, nous comparerons la situation de ceux ayant été formés dans le cadre de projets de recherche impliquant une collaboration université-milieu à celle des étudiants plus «traditionnels » qui n'ont pas vécu de tels partenariats au cours de leur cheminement.

\section{L'ENQUÊTE}

Le projet de recherche sur la formation aux cycles supérieurs dans le contexte des relations université-milieu a débuté au Centre interuniversitaire de recherche sur la science et la technologie (CIRST) en juin 2002 grâce au soutien du programme d'Initiative de la nouvelle économie du Conseil de recherche en sciences humaines du Canada. Le Fonds québécois de recherche sur la nature et les technologies et le Fonds québécois de recherche sur la société et la culture ont également soutenu le projet, notamment pour son volet portant sur les Bourses de recherche en milieu de pratique.

Après une période exploratoire au cours de laquelle une vingtaine d'étudiants, de diplômés, de professeurs et d'administrateurs universitaires ont été rencontrés ${ }^{2}$, un questionnaire détaillé a été construit afin de recueillir des données sur toutes les étapes de la formation à la recherche à la maîtrise et au doctorat. Les conditions d'études, la direction, l'encadrement, le financement, le sujet, les conditions de collaboration avec le milieu (s'il y a lieu), la diffusion des résultats de recherche, le bilan, la durée des études et les projets professionnels (pour les étudiants) ou l'insertion professionnelle (pour les diplômés) y ont été abordés.

Le questionnaire a d'abord été administré par Internet aux boursiers du programme de "Bourses de recherche en milieu de pratique» des Fonds québécois de recherche (FQRNT, FQRSC et FRSQ) au cours de l'hiver 2003 puis, après des corrections et modifications mineures, aux étudiants du Régime de partenariat de l’Université de Sherbrooke.

1. Seuls les programmes de maîtrise et de doctorat de type «recherche», c'est-à-dire comprenant la rédaction d'un mémoire ou d'une thèse, sont visés. Les programmes de type "professionnel» comportant un stage et/ou un essai ne font pas partie de notre échantillon.

2. Cette phase exploratoire a été réalisée conjointement par une équipe du CIRST et un assistant de recherche embauché par le Conseil national des cycles supérieurs. 
Par la suite, l'ensemble des étudiants et diplômés des universités québécoises francophones ont été sollicités pour participer à l'enquête. Les participants ont été recrutés de diverses manières: affichage, annonces dans les médias institutionnels, diffusion électronique, contacts avec les programmes d'études, etc. Afin de rejoindre un plus grand nombre d'étudiants impliqués dans des projets en collaboration université-milieu, nous avons également constitué des listes de chercheurs à partir des données sur le financement de la recherche ainsi que d'autres sources de données. Les étudiants ont pu remplir le questionnaire de manière confidentielle et sécuritaire entre les mois de novembre 2003 et juin 2004. Au total, 956 répondants ont fourni un questionnaire complet, dont 104 boursiers du programme «en milieu de pratique», 9 étudiants du Régime de partenariat et 843 autres. Ces répondants sont répartis entre les diverses universités francophones ${ }^{3}$ et entre les disciplines.

Pour faciliter la présentation des résultats dans cette analyse, nous avons divisé les répondants en trois catégories: les boursiers en milieu de pratique des Fonds de recherche québécois (appelés ci-après les «boursiers»), les autres étudiants et diplômés affirmant avoir été impliqués dans un projet de recherche en collaboration université-milieu et les autres étudiants et diplômés n'ayant pas été impliqués dans un tel projet. Le tableau 6.1 montre la distribution des répondants selon les catégories et les disciplines. Il est nécessaire de réaliser une analyse séparée par grands groupes disciplinaires, car la structure et l'expérience de la formation aux cycles supérieurs varie de manière importante d'une discipline à l'autre (Delamont, Parry et Atkinson, 2000; Becher et Trowler, 2001). Comme on peut le voir, le nombre de répondants en sciences humaines et en sciences de la santé chez les boursiers était faible et rend difficiles les comparaisons entre les différents types d'étudiants. Ainsi, dans cet article, seules les données concernant les répondants des sciences naturelles et du génie seront considérés. L'analyse du cas spécifique des sciences humaines et sociales, cependant, demeure d'une grande importance et devrait faire l'objet de recherches plus approfondies étant donné l'effet important que semble avoir la participation à des collaborations de recherche sur l'expérience étudiante dans ces disciplines.

3. Certains répondants sont issus des universités anglophones, mais ils constituent moins de $5 \%$ des répondants de l'enquête. Le questionnaire n'étant pas traduit en anglais, nous n'avons pu recruter systématiquement au sein de ces institutions. 
TABLEAU 6.1

Distribution des répondants par catégorie

\begin{tabular}{|l|c|c|c|c|}
\hline & $\begin{array}{c}\text { Boursiers en milieu } \\
\text { de pratique }\end{array}$ & $\begin{array}{c}\text { Autres } \\
\text { collaborateurs }\end{array}$ & Non-collaborateurs & Total \\
\hline Sciences naturelles et génie & $72,1 \%(75)$ & $38,1 \%(192)$ & $31,3 \%(109)$ & $39,3 \%(376)$ \\
\hline Sciences de la santé & $11,5 \%(12)$ & $19,8 \%(100)$ & $17,5 \%(61)$ & $18,1 \%(61)$ \\
\hline Sciences humaines et sociales & $14,4 \%(15)$ & $39,9 \%(201)$ & $37,6 \%(131)$ & $36,3 \%(347)$ \\
\hline Total & $100 \%(102)$ & $100 \%(493)$ & $100 \%(301)$ & $100 \%(896 *)$ \\
\hline
\end{tabular}

* Le total des répondants est égal à 956, mais, faute de réponses à certaines questions, certains n'ont pu être classés.

\section{RÉSULTATS}

Deux parties de notre questionnaire traitaient de l'insertion professionnelle: la première s'adressait aux étudiants actuels et les interrogeait sur leurs projets professionnels, tandis que la seconde s'adressait aux diplômés et recueillait de l'information sur l'emploi occupé après les études. Le portrait qui se détache des différentes catégories de répondants est nettement contrasté entre ceux qui ont participé à des projets de recherche en collaboration et ceux qui ont fait des recherches appartenant essentiellement au champ universitaire.

\section{Les projets professionnels}

Les collaborateurs et les non-collaborateurs se distinguent très significativement quant à leurs aspirations professionnelles. En effet, quand on leur demande dans quel secteur ils préféreraient œuvrer au terme de leurs études, les portraits sont pratiquement inversés: seulement un boursier du domaine scientifique sur cinq affirme préférer travailler «dans une université » tandis que c'est le cas d'un étudiant sur deux parmi les noncollaborateurs. Cette différence notoire n'est pas le fait d'une plus grande proportion d'étudiants à la maîtrise parmi les boursiers et de doctorants parmi les non-collaborateurs, puisque la répartition par cycle est identique dans ces deux catégories. À mi-chemin entre ces deux extrêmes, on trouve les autres collaborateurs, dont le tiers préféreraient travailler à l'université. 
TABleau 6.2

Projets professionnels des étudiants "L'emploi que vous aimeriez le plus occuper dans I'avenir serait-il plutôt..."

\begin{tabular}{|l|c|c|c|c|}
\hline & $\begin{array}{c}\text { Boursiers en milieu } \\
\text { de pratique }\end{array}$ & $\begin{array}{c}\text { Autres } \\
\text { collaborateurs }\end{array}$ & Non-collaborateurs & Total \\
\hline$\ldots$ dans une entreprise. & $40,0 \%(26)$ & $23,3 \%(35)$ & $23,6 \%(21)$ & $27,0 \%(82)$ \\
\hline$\ldots$ dans une université. & $18,5 \%(12)$ & $32,7 \%(49)$ & $50,6 \%(45)$ & $34,9 \%(106)$ \\
\hline $\begin{array}{l}\text {... dans une institution } \\
\text { gouvernementale ou } \\
\text { paragouvernementale. }\end{array}$ & $26,2 \%(17)$ & $30,0 \%(45)$ & $12,4 \%(11)$ & $24,0 \%(73)$ \\
\hline $\begin{array}{l}\ldots . \text { dans le milieu } \\
\text { communautaire. }\end{array}$ & $0,0 \%(0)$ & $2,0 \%(3)$ & $3,4 \%(3)$ & $2,0 \%(6)$ \\
\hline Autre. & $4,6 \%(3)$ & $5,3 \%(8)$ & $5,6 \%(5)$ & $5,3 \%(16)$ \\
\hline Je ne sais pas. & $10,8 \%(7)$ & $6,7 \%(10)$ & $4,5 \%(4)$ & $6,9 \%(21)$ \\
\hline Total & $100 \%(65)$ & $100 \%(150)$ & $100 \%(89)$ & $100 \%(304)$ \\
\hline
\end{tabular}

Que souhaitent donc faire les boursiers? La plupart perçoivent l'entreprise comme le milieu de travail idéal, et quelques-uns songent à intégrer la fonction publique ou parapublique. La tendance à privilégier l'entreprise est peut-être liée au fait que les boursiers collaborent majoritairement avec des entreprises au cours de leurs études. L'autre fait marquant observable dans le tableau est la très faible proportion de non-collaborateurs intéressés à travailler dans des organisations gouvernementales ou paragouvernementales. Les perspectives d'emploi dans ce domaine n'intéressent pas les étudiants formés de manière traditionnelle, ou peut-être ignorent-ils les types d'emplois qui y sont offerts.

Peu importe le secteur dans lequel ils veulent œuvrer, la grande majorité des étudiants ont l'intention de faire de la recherche dans l'avenir: moins de $10 \%$ ne souhaitent faire que peu ou pas de recherche. La moitié des répondants, peu importe leur catégorie, souhaitent partager leur temps entre la recherche et d'autres tâches, et environ $40 \%$ souhaitent faire surtout de la recherche. On observe donc que le fait d'être un boursier ou d'avoir entretenu un autre type de collaboration avec des partenaires non universitaires au cours des études a certes une influence sur le milieu de travail souhaité par les étudiants, mais non sur leur désir de faire de la recherche. On peut néanmoins penser que le type de recherche qu'ils souhaitent faire est peut-être différent. 
TABLEAU 6.3

Intention de faire de la recherche "Quelle place souhaitez-vous que la recherche occupe dans votre carrière? »

\begin{tabular}{|l|c|c|c|c|}
\hline & $\begin{array}{c}\text { Boursiers en milieu } \\
\text { de pratique }\end{array}$ & $\begin{array}{c}\text { Autres } \\
\text { collaborateurs }\end{array}$ & Non-collaborateurs & Total \\
\hline Pas de recherche. & $0,0 \%(0)$ & $0,7 \%(1)$ & $2,2 \%(2)$ & $1,0 \%(3)$ \\
\hline $\begin{array}{l}\text { Un peu de recherche mais } \\
\text { surtout d'autres tâches. }\end{array}$ & $7,6 \%(5)$ & $9,3 \%(14)$ & $8,9 \%(8)$ & $8,8 \%(27)$ \\
\hline $\begin{array}{l}\text { Partager mon temps entre la } \\
\text { recherche et d'autres tâches. }\end{array}$ & $45,5 \%(30)$ & $51,7 \%(78)$ & $48,9 \%(44)$ & $49,5 \%(152)$ \\
\hline $\begin{array}{l}\text { Surtout de la recherche et } \\
\text { quelques autres tâches. }\end{array}$ & $42,4 \%(28)$ & $33,1 \%(50)$ & $33,3 \%(30)$ & $35,2 \%(108)$ \\
\hline Seulement de la recherche. & $4,5 \%(3)$ & $3,3 \%(5)$ & $4,4 \%(4)$ & $3,9 \%(12)$ \\
\hline Je ne sais pas. & $0,0 \%(0)$ & $2,0 \%(3)$ & $2,2 \%(2)$ & $1,6 \%(5)$ \\
\hline Total & $100 \%(66)$ & $100 \%(151)$ & $100 \%(90)$ & $100 \%(307)$ \\
\hline
\end{tabular}

Cependant, les étudiants sont nettement distingués par une autre variable: leur sentiment d'être prêts à affronter le marché du travail. Les boursiers scientifiques du programme de recherche en milieu de pratique se sentent significativement mieux préparés que les autres à entrer sur le marché du travail, tandis que les collaborateurs non boursiers et les noncollaborateurs se sentent moins préparés. Comme on peut l'observer au tableau 6.4, les boursiers et même les autres collaborateurs sont plus nombreux à se dire "très bien préparés », et pratiquement aucun d'entre eux ne se dit «très mal préparé » à faire face aux défis qui les attendent après leurs études. Il est important de souligner que ce sont les boursiers qui n'ont pas l'intention d'œuvrer en milieu universitaire dans l'avenir qui se sentent les mieux préparés $(3,45)$ au marché du travail.

En ce qui a trait aux étudiants qui n'ont pas encore terminé leurs études, il ressort donc clairement que les boursiers en milieu de pratique ont des projets de carrière plus diversifiés et se sentent mieux préparés à affronter le marché du travail, dans le domaine scientifique à tout le moins, que leurs collègues non collaborateurs. Les étudiants ayant fait des recherches en collaboration sans bourse se trouvent pour leur part souvent à mi-chemin entre les deux pôles, en fonction de l'intensité de leur partenariat avec l'organisation non universitaire. Il ne s'agit toutefois là que de projets. Nous verrons maintenant ce qu'il advient effectivement des diplômés. 
TABLEAU 6.4

Sentiment de préparation à l'égard du marché du travail Étudiants "Comment vous sentez-vous par rapport à votre entrée sur le marché du travail ? "

\begin{tabular}{|l|c|c|c|c|}
\hline & $\begin{array}{c}\text { Boursiers en milieu } \\
\text { de pratique }\end{array}$ & $\begin{array}{c}\text { Autres } \\
\text { collaborateurs }\end{array}$ & Non-collaborateurs & Total \\
\hline Très mal préparé. & $0,0 \%(0)$ & $2,7 \%(4)$ & $4,7 \%(4)$ & $2,7 \%(8)$ \\
\hline Plutôt mal préparé. & $7,6 \%(5)$ & $15,4 \%(23)$ & $15,1 \%(13)$ & $13,6 \%(41)$ \\
\hline Plutôt bien préparé. & $57,6 \%(38)$ & $55,7 \%(83)$ & $65,1 \%(56)$ & $58,8 \%(177)$ \\
\hline Très bien préparé. & $34,8 \%(23)$ & $26,2 \%(39)$ & $15,1 \%(13)$ & $24,9 \%(75)$ \\
\hline Moyenne & $3,27 / 4$ & $3,05 / 4$ & $2,91 / 4$ & $3,06 / 4$ \\
\hline
\end{tabular}

\section{L'insertion professionnelle effective}

Parmi l'ensemble de nos répondants en sciences naturelles et génie, 67 étaient diplômés au moment de remplir le questionnaire. Parmi ces derniers, 37 possédaient une maîtrise et 30, un doctorat. Il est difficile de comparer la situation des boursiers à celle des autres catégories quant à leur insertion professionnelle, car seuls quatre d'entre eux occupent un emploi, les quatre autres diplômés ayant indiqué avoir poursuivi leurs études au doctorat après leur maîtrise en milieu de pratique. Leur profil étant comparable à celui des autres collaborateurs, nous joindrons donc les deux catégories (boursiers et autres collaborateurs) pour les besoins de cette section. Nous analysons également simultanément la situation des diplômés de la maîtrise et du doctorat, car leur nombre est trop faible pour autoriser de véritables comparaisons.

TABLEAU 6.5

\section{Occupation des répondants}

"Lequel des énoncés suivants s'applique le mieux à votre situation? »

\begin{tabular}{|l|c|c|c|c|}
\hline & $\begin{array}{c}\text { Boursiers en milieu } \\
\text { de pratique }\end{array}$ & $\begin{array}{c}\text { Autres } \\
\text { collaborateurs }\end{array}$ & Non-collaborateurs & Total \\
\hline J'occupe un emploi. & $50,0 \%(4)$ & $89,2 \%(33)$ & $89,5 \%(17)$ & $84,4 \%(54)$ \\
\hline $\begin{array}{l}\text { Je n'occupe pas d'emploi mais } \\
\text { j'en cherche un. }\end{array}$ & $0,0 \%(0)$ & $10,8 \%(4)$ & $5,3 \%(1)$ & $7,8 \%(1)$ \\
\hline $\begin{array}{l}\text { Je n'occupe pas d'emploi et je } \\
\text { n'en cherche pas. }\end{array}$ & $50,0 \%(4)$ & $0,0 \%(4)$ & $5,3 \%(1)$ & $7,8 \%(5)$ \\
\hline Total & $100 \%(8)$ & $100 \%(37)$ & $100 \%(19)$ & $100 \%(64 \%)$ \\
\hline
\end{tabular}

* Le nombre n'est pas égal à 67 en raison des non-réponses. 
Parmi ceux qui sont présentement en emploi, les tendances observées auprès des étudiants se maintiennent: les collaborateurs sont beaucoup plus nombreux à travailler dans le secteur privé, en entreprise, tandis que les non-collaborateurs œuvrent majoritairement en milieu universitaire. Tous n'ont toutefois pas précisé le type d'emploi qu'ils occupent et nous savons que seuls certains, parmi ceux qui ont un poste universitaire, travaillent comme professeurs, alors que d'autres occupent un poste d'assistant de recherche ou de chercheur contractuel.

TABLeau 6.6

Milieu de travail «À laquelle des catégories suivantes appartient votre employeur actuel?»

\begin{tabular}{|l|c|c|c|}
\hline & Collaborateurs & Non-collaborateurs & Total \\
\hline Entreprise & $44,7 \%(17)$ & $17,6 \%(3)$ & $36,4 \%(20)$ \\
\hline Université & $28,9 \%(11)$ & $52,9 \%(9)$ & $36,4 \%(20)$ \\
\hline Ministère ou organisme gouvernemental & $21,1 \%(8)$ & $5,9 \%(1)$ & $16,4 \%(9)$ \\
\hline Autre établissement d'enseignement & $0,0 \%(0)$ & $17,6 \%(3)$ & $5,5 \%(3)$ \\
\hline Organisme non gouvernemental ou communautaire & $2,6 \%(1)$ & $5,9 \%(1)$ & $3,6 \%(2)$ \\
\hline Autre & $2,6 \%(1)$ & $0,0 \%(0)$ & $1,8 \%(1)$ \\
\hline Total & $100 \%(38)$ & $100 \%(17)$ & $100 \%(55)$ \\
\hline
\end{tabular}

En règle générale, comme l'indique le tableau 6.7, les boursiers et les autres collaborateurs semblent s'être trouvé du travail plus rapidement que leurs collègues qui n'ont pas participé à des recherches en collaboration. En fait, plusieurs avaient déjà un emploi avant leurs études, qu'ils ont conservé par la suite. C'est notamment le cas de plusieurs diplômés du programme «Régime de partenariat» de l'Université de Sherbrooke. Dans ce programme, les étudiants conservent leur emploi au sein d'une organisation non universitaire au cours de leurs études et doivent consacrer leur mémoire ou leur thèse à un problème de recherche proche des besoins de leur employeur. Les autres collaborateurs ont généralement trouvé un emploi avant même la fin de leurs études, ce qui est moins souvent le cas des non-collaborateurs. 
L'exploration approfondie des données du questionnaire nous force cependant à constater que l'insertion professionnelle des jeunes chercheurs n'est pas un processus linéaire simple. Par exemple, parmi les répondants ayant indiqué «ne pas encore avoir trouvé d'emploi» (tableau 6.7), il s'en trouve un qui a par ailleurs indiqué avoir un emploi dans une université. De même, ceux qui ont dit avoir «poursuivi d'autres études immédiatement après» déclarent aussi parfois occuper un poste dans une université, une entreprise ou un organisme gouvernemental. Les périodes de formation et d'insertion professionnelle semblent donc, chez certains étudiants à tout le moins, superposées.

\section{TABLEAU 6.7}

\section{Temps requis pour l'insertion professionnelle}

"Combien de temps s'est écoulé entre le moment où vous avez obtenu votre diplôme et le moment où vous avez trouvé un emploi ?»

\begin{tabular}{|l|c|c|c|}
\hline & Collaborateurs & Non-collaborateurs & Total \\
\hline J'ai le même emploi qu'au début... & $16,7 \%(8)$ & $5,3 \%(1)$ & $13,4 \%(9)$ \\
\hline J'ai trouvé un emploi avant la fin... & $43,8 \%(21)$ & $31,6 \%(6)$ & $40,3 \%(27)$ \\
\hline Moins d'un mois. & $4,2 \%(2)$ & $5,3 \%(1)$ & $4,5 \%(3)$ \\
\hline De 1à 5 mois. & $14,6 \%(7)$ & $15,8 \%(3)$ & $14,9 \%(10)$ \\
\hline De 6à 12 mois. & $4,2 \%(2)$ & $5,3 \%(1)$ & $4,5 \%(3)$ \\
\hline Plus de 12 mois. & $0,0 \%(0)$ & $10,5 \%(2)$ & $3,0 \%(2)$ \\
\hline Je n'ai pas encore trouvé d'emploi. & $4,2 \%(2)$ & $5,3 \%(1)$ & $4,5 \%(3)$ \\
\hline J'ai poursuivi d'autres études immédiatement après. & $4,2 \%(2)$ & $21,1 \%(4)$ & $9,0 \%(6)$ \\
\hline Total & $100 \%(44)$ & $100 \%(19)$ & $100 \%(63)$ \\
\hline
\end{tabular}

Nous avons vu plus tôt que les boursiers qui étaient toujours étudiants se sentaient mieux préparés que les autres à affronter le marché du travail. Cet optimisme survit-il à l'expérience effective de l'emploi? Si c'est vrai pour les (rares) boursiers diplômés, qui se disent tous bien préparés, il n'en va pas de même pour les autres collaborateurs. Mis ensemble, les collaborateurs (BMP et autres) disent, en moyenne, avoir été moins bien préparés que les diplômés non collaborateurs. La différence n'est toutefois pas significative entre les deux groupes. 
TABLEAU 6.8

Sentiment de préparation à l'égard du marché du travail Diplômés "Selon vous, comment votre programme vous a-t-il préparé, jusqu'à présent, pour le marché du travail ?»

\begin{tabular}{|l|c|c|c|}
\hline & Collaborateurs & Non-collaborateurs & Total \\
\hline Très mal préparés. & $4,2 \%(2)$ & $10,5 \%(2)$ & $6,0 \%(4)$ \\
\hline Plutôt mal préparés. & $16,7 \%(8)$ & $5,3 \%(1)$ & $13,4 \%(9)$ \\
\hline Plutôt bien préparés. & $50,0 \%(24)$ & $36,8 \%(7)$ & $46,3 \%(31)$ \\
\hline Très bien préparés. & $27,1 \%(13)$ & $42,1 \%(8)$ & $31,3 \%(21)$ \\
\hline Moyenne & $3,02 / 4$ & $3,17 / 4$ & $3,06 / 4$ \\
\hline
\end{tabular}

\section{CONCLUSION}

Le fait de réaliser ses études de maîtrise ou de doctorat en collaboration avec une ou plusieurs organisation(s) non universitaire(s) expose celle ou celui qui s'y prête à une réalité potentiellement très différente de celle des étudiantes et étudiants formés en contexte traditionnel. La collaboration ne prend pas toujours la même forme et ses effets sont généralement proportionnels à son intensité. Par exemple, le seul fait de recevoir du financement provenant d'une organisation non universitaire, sans avoir de véritables interactions avec les représentants de cette dernière, procure une expérience différente de celle découlant de contacts quotidiens et d'échanges importants avec des intervenants issus de l'extérieur du milieu institutionnel. On peut penser que plus l'intensité du partenariat est grande, plus les étudiants intègrent de nouvelles manières de faire et de percevoir à leurs propres pratiques de recherche en développement.

Sans surprise, ces changements ont des conséquences sur l'insertion professionnelle, autant prévue qu'effective, des étudiants. Chez les boursiers du programme de recherche en milieu de pratique, qui ont en moyenne les collaborations les plus intenses avec leurs partenaires non universitaires, on observe un plus grand intérêt pour les carrières de recherche non universitaires et un plus grand sentiment de préparation au marché du travail. Ces aspirations se traduisent concrètement par une insertion professionnelle plus rapide pour les étudiants qui collaborent avec des organisations du milieu au cours de leurs études. D'ailleurs, la rapidité avec laquelle certains se trouvent un emploi laisse présager qu'ils avaient déjà commencé à faire des démarches sérieuses 
au cours de leurs études, ou même qu'ils avaient déjà été approchés par un ou des employeurs potentiels. Les autres étudiants collaborateurs, mais non boursiers, n'ont pas toutes les caractéristiques des boursiers en milieu de pratique mais ont tout de même des projets professionnels nettement plus diversifiés que leurs collègues non collaborateurs, et se trouvent aussi des emplois plus rapidement. Ils ne se disent toutefois pas aussi préparés au marché du travail, un phénomène que nous examinerons plus tard dans le cadre d'entrevues. Qui plus est, à l'heure actuelle, les diplômés sont encore très peu nombreux au sein de nos répondants. La relance que nous comptons effectuer en 2006 nous permettra de voir où en sont rendus les étudiants de la première enquête afin de mieux saisir l'effet de la participation à des collaborations de recherche sur l'insertion professionnelle ultérieure. Le plus grand nombre de répondants diplômés nous permettra également de mieux comprendre les liens entre certaines caractéristiques de la formation expérimentée par les étudiants et leur emploi par la suite.

D'ici là, force est de reconnaître l'effet des collaborations de recherche université-milieu sur les étudiants qui les expérimentent. Un facteur important attire notre attention comme piste d'explication provisoire: l'ouverture du réseau social. En effet, nous avons observé que les étudiants engagés dans des partenariats université-milieu reçoivent le soutien d'un plus grand nombre d'intervenants différents dans la réalisation de leur mémoire ou de leur thèse. Comme le montre le tableau 6.9, les trois quarts des boursiers et plus de la moitié des autres collaborateurs reçoivent le soutien de quatre catégories d'acteurs différents et plus. En outre, dans sensiblement le même nombre de cas, on trouve au moins un intervenant non universitaire (chercheur ou non-chercheur) au nombre des personnes ayant fourni de l'aide ou du soutien à l'étudiant au cours de son cheminement. De telles interactions directes avec des acteurs du milieu montrent que les étudiants sont, avant même d'entrer formellement sur le marché du travail, en contact avec des employeurs potentiels qu'ils ont déjà appris à connaître, et auprès desquels ils ont commencé à se faire connaître. Il n'est donc pas surprenant que, d'une part, les étudiants collaborateurs soient plus enclins à s'intéresser à différentes carrières, ne serait-ce que parce qu'ils sont exposés à divers modèles professionnels, et que, d'autre part, ils se trouvent plus rapidement du travail une fois leurs études terminées. 
TABLEAU 6.9

Nombre et catégories d'acteurs soutenant l'étudiant dans son mémoire (sa thèse) «Lesquelles des personnes suivantes vous ont fourni de l'aide ou du soutien dans la réalisation de votre projet de recherche* ?"

\begin{tabular}{|l|c|c|c|c|}
\hline & $\begin{array}{c}\text { Boursiers en milieu } \\
\text { de pratique }\end{array}$ & $\begin{array}{c}\text { Autres } \\
\text { collaborateurs }\end{array}$ & Non-collaborateurs & Total \\
\hline Nombre de catégories \\
\hline 3 et moins & $25,3 \%(19)$ & $43,8 \%(84)$ & $58,7 \%(64)$ & $44,4 \%(167)$ \\
\hline 4 et plus & $73,3 \%(55)$ & $56,3 \%(108)$ & $38,5 \%(42)$ & $54,5 \%(205)$ \\
\hline Types d'intervenants & \multicolumn{5}{|l|}{} \\
\hline Comprend des universitaires. & $96,0 \%(72)$ & $99,5 \%(191)$ & $97,2 \%(106)$ & $98,1 \%(369)$ \\
\hline $\begin{array}{l}\text { Comprend des non- } \\
\text { universitaires. }\end{array}$ & $80,0 \%(60)$ & $56,8 \%(109)$ & $5,5 \%(6)$ & $46,5 \%(175)$ \\
\hline
\end{tabular}

* Le choix de réponse comprenait: directeur, codirecteur, autres professeurs, chercheur d'organisation non universitaire, non-chercheur d'organisation non universitaire, professionnel de recherche à l'université, technicien à l'université, étudiants plus avancés, étudiants de niveau équivalent ou moindre, autre personne.

La formation en milieu de pratique, bien qu'elle ait ses avantages, ne constitue pas pour autant une panacée en matière d'insertion professionnelle des jeunes chercheurs, ne serait-ce que parce que, à l'heure actuelle du moins, il ne s'agit pas d'un mode de formation qui s'adapte à toutes les problématiques de recherche. Les effets de la participation à la recherche en milieu de pratique sur la carrière de ceux qui choisiraient de suivre la voie du travail universitaire sont également à déterminer. Certes, nous savons que les pratiques de collaboration n'affectent pas significativement la propension des étudiants à participer à des publications évaluées par les pairs, mais l'habitus scientifique comprend beaucoup d'autres aspects qui ne peuvent tous être mesurés simplement mais qui sont néanmoins reconnus par les comités d'embauche des départements. Les étudiants ayant expérimenté la formation en milieu de pratique en seront-ils aussi dotés que les autres candidats au professorat? Seul le suivi longitudinal de notre cohorte nous permettra de l'estimer.

Quant aux jeunes chercheurs qui seront embauchés à l'extérieur $\mathrm{du}$ milieu universitaire, il ne serait pas surprenant qu'ils contribuent au maintien de projets de recherche en collaboration et à l'établissement de nouvelles initiatives semblables (Grossetti et Bès, 2001). Au cours d'entrevues exploratoires, puis par le biais de questions ouvertes dans le questionnaire, plusieurs étudiants nous ont confié leur volonté de devenir des interfaces entre le monde universitaire et son environne- 
ment, notamment industriel. Nous l'avons vu, la majorité des étudiants collaborateurs n'ont pas le projet de travailler à l'université, mais cela ne les empêche pas de vouloir garder leurs liens avec leur ancien directeur et leur ancienne équipe, d'autant plus qu'il est probable qu'ils demeurent actifs en recherche. Ce faisant, ces anciens étudiants deviendront potentiellement des ambassadeurs pour le monde universitaire dans l'industrie, ou vice-versa. S'il est attendu que ces pratiques de formation transforment le monde universitaire, il est également probable qu'elles transforment aussi les entreprises et les gouvernements, rendant ces derniers plus aptes à dialoguer avec les chercheurs universitaires et à intégrer les résultats de la recherche universitaires à leurs pratiques.

En somme, l'émergence d'une nouvelle espèce de chercheurs, au sein desquels coïncideraient habitus universitaire et propension à s'adapter aux besoins du milieu, sera sans doute favorisée par la multiplication d'opportunités de formation en milieu de pratique. À court terme, cette tendance se traduit déjà par un passage sensiblement facilité des études au marché du travail. Les effets à plus long terme, tant sur les trajectoires de chercheurs que sur les institutions universitaires et non universitaires, devront pour leur part continuer de faire l'objet de recherches qui, elles-mêmes, devront bénéficier de la collaboration entre les différents milieux.

\section{BIBLIOGRAPHIE}

Becher, T. et R. Trowler (2001). Academic Tribes and Territories, $2^{\mathrm{e}}$ éd., Buckingham, Society for Research Into Higher Education et Open University Press.

Delamont, S., O. Parry et P. AtKinson (2000). The Doctoral Experience: Success and Failure in Graduate School, Londres, Falmer Press.

Gemme, B. et Y. Gingras (à paraître). Les effets de la formation en milieu de pratique à la maîtrise et au doctorat, Note de recherche du CIRST.

Gemme, B. et Y. Gingras (à paraître). «The New Production of Researchers » soumis pour publication dans le livre de A. Chan et D. Fisher, Transforming Academic Culture, Vancouver, UBC Press.

Gingras, Y., B. Godin et M. Trépanier (1999). «La place des universités dans les politiques scientifiques et technologiques canadiennes et québécoises » dans Paul Beaulieu et Denis Bertrand (dir.), L'État québécois et les universités: acteurs et enjeux, Sainte-Foy, Presses de l'Université du Québec, p. 69-99. 
Godin, B., M. Trépanier et M. Albert (2000). «Des organismes sous tension: les conseils subventionnaires et la politique scientifique», Sociologie et sociétés, $X X X I I(1)$, p. 17-42.

Grossetti, M. et M.-P. BÈs (2001). «Encastrements et découplages dans les relations science-industrie», Revue française de sociologie, 42(2), p. 327-355.

Slaughter, S. et L.L. Leslie (1997). Academic Capitalism: Politics, Policies and the Entrepreneurial University, Baltimore, Johns Hopkins University Press. 


\title{
Chapitre
}

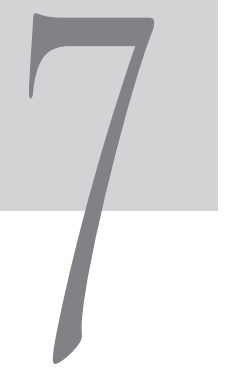

\section{EXODE DES CERVEAUX \\ Phénomène imaginaire?}

\author{
Stéphanie Andrew et Daniel Parent \\ Département d'économie Université McGill
}

Peu de questions suscitent autant d'inquiétudes et mobilisent autant d'énergie dans le milieu universitaire que le problème du recrutement et de la rétention de professeurs qualifiés, à tout le moins dans certains départements. La même problématique est présente dans d'autres secteurs employant des travailleurs hautement qualifiés. L'attrait apparemment irrésistible des salaires élevés et des faibles taux de taxation en vigueur aux États-Unis, en plus des conditions de travail autres que salariales $^{1}$, est perçu comme posant un problème qui ne peut que s'aggraver à moins que des mesures vigoureuses ne soient mises en place.

Par ailleurs, lorsqu'on évoque la question de l'exode des cerveaux, plusieurs soulignent que le problème est plus imaginaire que réel, car, après tout, un volume considérable d'individus fortement scolarisés choisissent d'immigrer au Canada: le nombre de ces immigrants surpasse

1. Par conditions de travail non salariales, pensons à la charge d'enseignement qui est typiquement réduite dans les universités dites de recherche (par opposition aux liberal arts colleges, qui sont par nature avant tout des institutions de premier cycle) ainsi qu'aux infrastructures de recherche en sciences appliquées, que ce soit dans le secteur universitaire ou dans les laboratoires de recherche à l'extérieur des universités. 
même le nombre de ceux qui quittent le pays (Zha, Drew et Scott Murray, 2000). De plus, le phénomène n'est pas nouveau: le Canada a toujours été un pays d'immigrants et d'émigrants. Par conséquent, plaider en faveur de politiques de nature essentiellement fiscales afin de contrer le départ des plus scolarisés est plutôt perçu comme faisant partie d'une stratégie déployée par des secteurs se percevant comme touchés, comme par exemple les universités, et visant à se voir accorder des faveurs fiscales au détriment d'autres groupes de la société qui sont peut-être davantage dans le besoin ${ }^{2}$.

Cet article cherche à contribuer à la question de savoir si l'exode des cerveaux est un phénomène imaginaire ou non en se penchant sur une catégorie de travailleurs qui devraient être particulièrement touchés, soit les détenteurs de doctorats. Nous examinerons d'abord les flux nets de migration de ces individus. Ensuite, et c'est ici que nous pensons que les pressions exercées par la force d'attraction du marché américain devraient se manifester si vraiment le phénomène de l'exode des cerveaux apparaît comme réel, nous nous pencherons sur les salaires relatifs des émigrants vers les États-Unis par rapport à ceux qui sont demeurés ici, ainsi que par rapport aux Américains de naissance ayant un doctorat.

\section{DESCRIPTION DE L'APPROCHE}

L'objectif poursuivi dans cet article est relativement simple et limité à la fois. Nous cherchons dans un premier temps à quantifier les flux migratoires nets des gens fortement scolarisés entre les années 1980 et 2000.

2. Par exemple, selon Yves Gingras du Département d'histoire de l'UQAM et directeur du Centre interuniversitaire de recherche sur la science et la technologie, «La pénurie de professeurs, c'est comme l'exode des cerveaux: ça n'existe pas» (La Presse, samedi, 14 février 2004). John Helliwell (Helliwell, 1999) documente le parcours des diplômés de l'Université de la Colombie-Britannique et trouve que la migration vers le sud était trois fois plus importante entre 1957-1961 qu'elle ne l'était au cours des années 1990. Il en conclut que bien que le phénomène apparaisse réel, il est probablement exagéré de s'en faire outre mesure et de mettre en place des politiques qui soient basées sur des inquiétudes en partie non fondées. Finalement, David Card (Card, 2003) documente également les flux migratoires des Canadiens aux États-Unis et note que le phénomène n'est pas nouveau. Un des résultats intéressants de Card est que contrairement à ce qui se passait il y a une vingtaine d'années, alors que les moins qualifiés s'expatriant aux États-Unis encouraient une pénalité salariale par rapport aux Américains de souche ayant les mêmes qualifications et que les plus qualifiés jouissaient au contraire d'une prime, les Canadiens émigrés en 2000 aux États-Unis sont au moins aussi bien payés en moyenne que les Américains, et ce, peu importe leur degré de qualification. Il note aussi que les salaires payés au Canada devraient s'ajuster en réaction à une recrudescence de l'émigration, si pénurie il y a. Or cet ajustement par les prix n’a pas encore eu lieu. 
En d'autres mots, nous voulons comparer le nombre de détenteurs de doctorats qui sont Canadiens de naissance et qui travaillent aux ÉtatsUnis $^{3}$. Ensuite, nous voulons faire la même analyse, mais dans le sens inverse, en examinant l'entrée de détenteurs de doctorat au Canada en provenance du reste du monde. Pour faire l'analyse des flux migratoires nets, nous utilisons les microdonnées des recensements canadiens effectués en 1981, 1991 et 1996, de même que les microdonnées des recensements américains de 1980, 1990 et 2000. Alors que les recensements canadiens nous permettent de quantifier les entrées de doctorants au Canada par année d'arrivée, les données américaines nous offrent la possibilité de faire de même pour quantifier les entrées en provenance du Canada.

L'intérêt de se pencher sur ces mouvements migratoires au cours de cette période est d'abord de potentiellement relativiser la nature du problème de l'exode des cerveaux, si problème il y $\mathrm{a}$. En effet, l'attirance des États-Unis pour les gens hautement qualifiés ne date pas d'hier étant donnél'explosion du réseau universitaire américain au cours de la première moitié $\mathrm{du} \mathrm{xx}^{\mathrm{e}}$ siècle et la demande en main-d'œuvre qui en a résulté. On pourrait même avancer sans trop se tromper que la qualité exceptionnelle de plusieurs universités américaines s'est bâtie d'une certaine façon aux dépens du reste du monde par la faculté qu'ont toujours eu les ÉtatsUnis à attirer les talents de pointe. Alors qu'au XIX ${ }^{\mathrm{e}}$ siècle, les universités

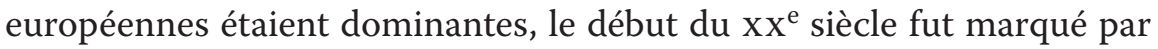
un déplacement de la recherche de pointe vers les États-Unis, déplacement sans nul doute aidé par le fait que l'Europe fut le théâtre de deux conflits mondiaux.

Bref, de tout temps ou presque, les Canadiens ont émigré vers les États-Unis et la question pertinente est alors d'examiner dans quelle mesure le phénomène s'est amplifié ou non au cours des dernières années.

3. À noter que nous pourrions aussi examiner l'émigration vers les États-Unis en provenance du Canada de ceux qui ne sont pas citoyens canadiens. Cette dernière catégorie de «cerveaux en exode» viserait simplement à tenir compte du nombre significatif de personnes œuvrant au Canada dans des secteurs faisant appel à des détenteurs de doctorat qui ne sont pas Canadiens et qui font bien sûr partie des gens à risque d'être attirés vers les États-Unis et qui, possiblement, utilise le Canada comme un stade intermédiaire avant d'aboutir aux États-Unis. Malheureusement, cette information n'est pas disponible dans tous les recensements. Nous nous en tiendrons donc aux citoyens canadiens. 
Dans un deuxième temps, nous cherchons à en savoir un peu plus sur ceux qui ont quitté le Canada pour aller travailler aux États-Unis ainsi que sur ceux qui sont entrés au Canada pour y travailler en examinant les salaires relatifs des différentes catégories (par exemple, le salaire des émigrants $v s$ celui des Canadiens demeurés au Canada). La raison pour laquelle nous utilisons le salaire est relativement simple. Lorsqu'on parle de l'exode des cerveaux, il est raisonnable de penser qu'on ne fait pas référence à des individus «moyens ». Ce qu'on a à l'esprit lorsqu'on s'inquiète du départ des travailleurs hautement qualifiés vers les États-Unis est bien davantage le problème du départ des «meilleurs». Or, comme c'est le cas dans beaucoup de secteurs, les meilleurs ont tendance à gagner des salaires plus élevés: de fait, il s'agit de l'une des raisons invoquées par ceux qui sont convaincus qu'il y a un problème pour expliquer le mouvement migratoire vers les États-Unis. Par conséquent, une comparaison des salaires des détenteurs de doctorat en provenance du Canada, qu'ils soient Canadiens ou non, avec les salaires de ceux qui sont demeurés ici devrait nous informer quant à la validité de cet argument. De plus, l'évolution des salaires relatifs au cours des vingt dernières années nous fournit une occasion de voir dans quelle mesure la nature et l'étendue du problème ont évolué au fil des ans.

Par ailleurs, il importe de souligner qu'il y a certaines des questions liées à la problématique de l'exode des cerveaux qu'il nous est impossible d'aborder, les possibilités offertes par les données des recensements étant limitées. Par exemple, un aspect important du phénomène de l'exode des cerveaux concerne les individus qu'on essaie de recruter dans les universités ou dans d'autres secteurs mais qui choisissent d'aller ailleurs, en général aux États-Unis ${ }^{4}$.

De plus, l'analyse des comparaisons salariales est limitative. Nul doute que maints facteurs non salariaux influencent la décision des individus de quitter le Canada ou de ne pas y venir. Pensons aux infrastructures de recherche et à la tâche d'enseignement, qui sont des facteurs très importants pour la plupart des chercheurs. Nous limitons notre analyse afin de circonscrire l'évolution d'un des facteurs jouant un rôle, soit le salaire.

4. À titre d'anecdote, le Département d'économique à McGill a dû faire paraître 10 offres à l'hiver 2003 pour combler 3 postes. Notre situation est loin d'être exceptionnelle si j'en juge par les échos provenant d'autres départements, à tout le moins en économique. Une question connexe concerne ceux qui ne se voient pas offrir de postes tout simplement parce qu'on perçoit qu'il n'y a que peu de chances de les voir accepter une offre. 


\section{RÉSULTATS}

\section{Données}

Les recensements américains et canadiens permettent de connaître le lieu de naissance de chaque résident, ainsi que l'année d'immigration s'il y a lieu. Le recensement américain de 2000 renseigne même sur le pays de provenance des immigrants entrés aux États-Unis au cours des cinq années précédentes. Par conséquent, il est possible de se faire une idée assez précise des flux d'émigration vers les États-Unis, que les émigrants soient Canadiens de naissance ou non. Les recensements antérieurs à 2000 ne permettent toutefois pas d'obtenir ce niveau de détail dans les données. Les recensements canadiens permettent également de connaître l'année d'arrivée de tous les immigrants. Ces données contiennent en outre de l'information sur le niveau de scolarité atteint, l'industrie, l'occupation, le nombre de semaines travaillées et le salaire annuel. Nous utilisons ces deux dernières variables afin de construire une mesure du salaire hebdomadaire dont nous faisons usage dans tous les tableaux. En ce qui concerne le niveau de scolarité complété, le seul problème potentiel concerne le recensement américain de 1980, lequel ne nous permet pas de savoir directement si le répondant détient un $\mathrm{Ph}$. D. ou non. Nous nous rabattons alors sur le fait que les répondants doivent déclarer le nombre d'années qu'ils ou elles ont passé à l'université (avec un diplôme à la fin du séjour) et attribuons le niveau de doctorat à ceux qui déclarent avoir passé huit ans et plus à l'université. Nul doute qu'il y a une part de «faux positifs» (ceux qui ont passé huit ans à l'université pour obtenir une maîtrise, par exemple, parce qu'ils ou elles ont travaillé à temps partiel durant les études) et de «faux négatifs» (ceux qui obtiennent un doctorat après quatre ou cinq ans à l'université) dans cette approximation, mais comme la question des années passées à l'université fait également référence à un diplôme sanctionnant la fin du séjour, le problème d'erreur de mesures ne devrait pas être trop grave. Notons finalement que nous limitons les échantillons aux personnes âgées entre 25 et 64 ans ayant travaillé au cours de l'année précédant l'année de recensement.

\section{Sommaire}

L'examen des flux nets révèle des fluctuations intéressantes au cours des dernières décennies. Tout d'abord, notons que peu importe que l'on examine tous les secteurs d'activité dans lesquels œuvrent les détenteurs de doctorats ou seulement le secteur de l'éducation, ils sont plus 
nombreux à immigrer au Canada quà en émigrer. Toutefois, alors que les flux nets étaient nettement plus favorables en 1970, le nombre relatif de détenteurs de doctorats émigrant vers les États-Unis s'est accru au fil des ans, tout particulièrement au cours des années 1990 et dans le secteur de l'éducation. Plusieurs facteurs concourraient à ce qu'il y ait un grand nombre d'immigrants hautement qualifiés qui aient immigré au Canada durant les années 1960 et 1970, mais un des plus importants a sans contredit été l'expansion rapide du réseau universitaire au Canada, plus particulièrement au Québec. Ce développement était bien sûr causé en partie par les changements démographiques induits par le baby-boom d'après-guerre. Toutefois, ce même facteur était également en jeu aux États-Unis à la même époque. La différence au Canada, particulièrement au Québec, était que la hausse du taux de fréquentation scolaire dans les universités était en pleine croissance tout au long des années 1960 et 1970 alors que le taux de fréquentation aux États-Unis a plafonné au début des années 1970.

Quant aux comparaisons de salaires, en général, nous trouvons que les émigrants en provenance du Canada bénéficient d'un avantage salarial substantiel par rapport à ceux qui demeurent au Canada. Cela est particulièrement vrai lorsqu'on ne se limite pas à ceux qui œuvrent dans le secteur de l'éducation. De plus, cet avantage salarial s'est accru considérablement au cours des années 1990 et cette augmentation ne peut s'expliquer uniquement par la détérioration du taux de change du dollar canadien durant cette période. Il semble vraiment y avoir une augmentation de la pression exercée par la hausse des salaires aux États-Unis au cours de la dernière décennie. Avec de tels écarts de salaires réels, il nous apparaît difficile de contrecarrer la migration des éléments talentueux vers le sud, à moins de mettre en place un ensemble de facteurs, salariaux ou «normatifs», qui rendent le Canada attrayant. Nous reviendrons plus loin sur la question des implications de ce que nous observons en termes de politique publique.

\section{Description détaillée}

Les figures 7.1 à 7.3 décrivent l'évolution des flux d'émigration et d'immigration par année d'arrivée, soit aux États-Unis dans le cas des émigrants, soit au Canada dans le cas des immigrants. Une première constatation nous semble assez évidente au vu des figures 7.1 et 7.2: ce n'est pas d'hier que les Canadiens fortement scolarisés choisissent d'émigrer vers les États-Unis. De fait, comme nous limitons notre analyse à ceux 
FIGURE 7.1A

Canadiens ayant un doctorat et travaillant aux É.-U.Personnes œuvrant dans tous les secteurs

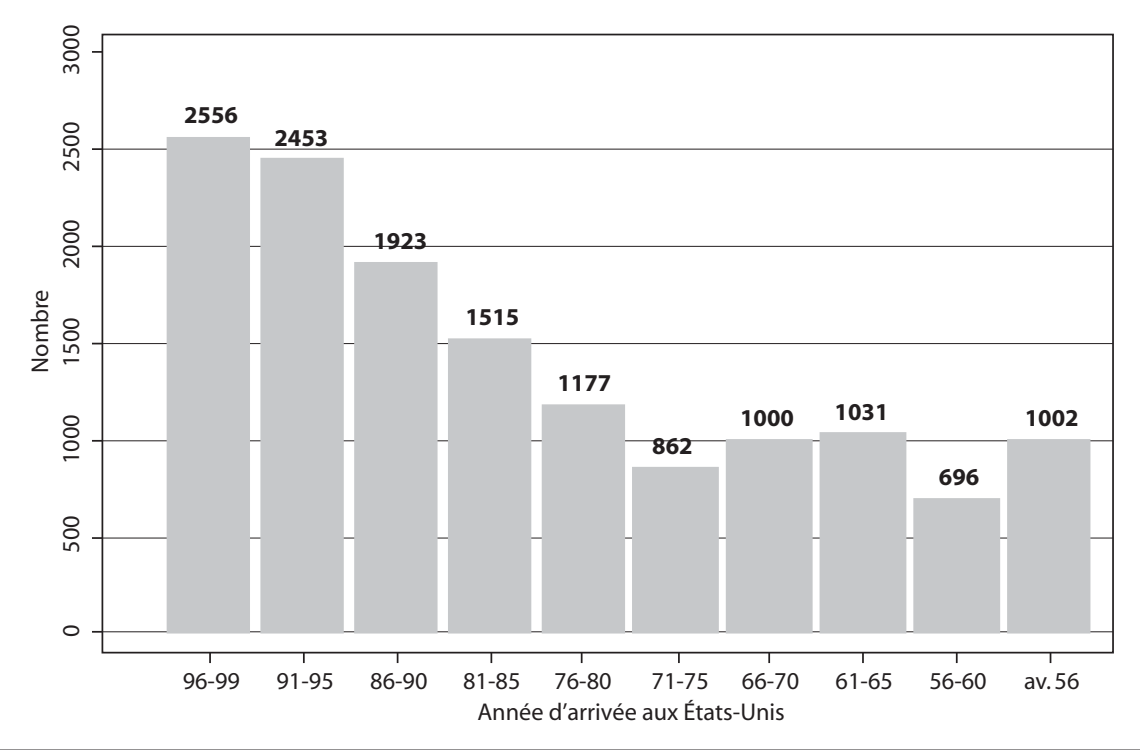

FIGURE 7.1B

Canadiens-français ayant un doctorat et travaillant aux É.-U.Personnes œuvrant dans tous les secteurs

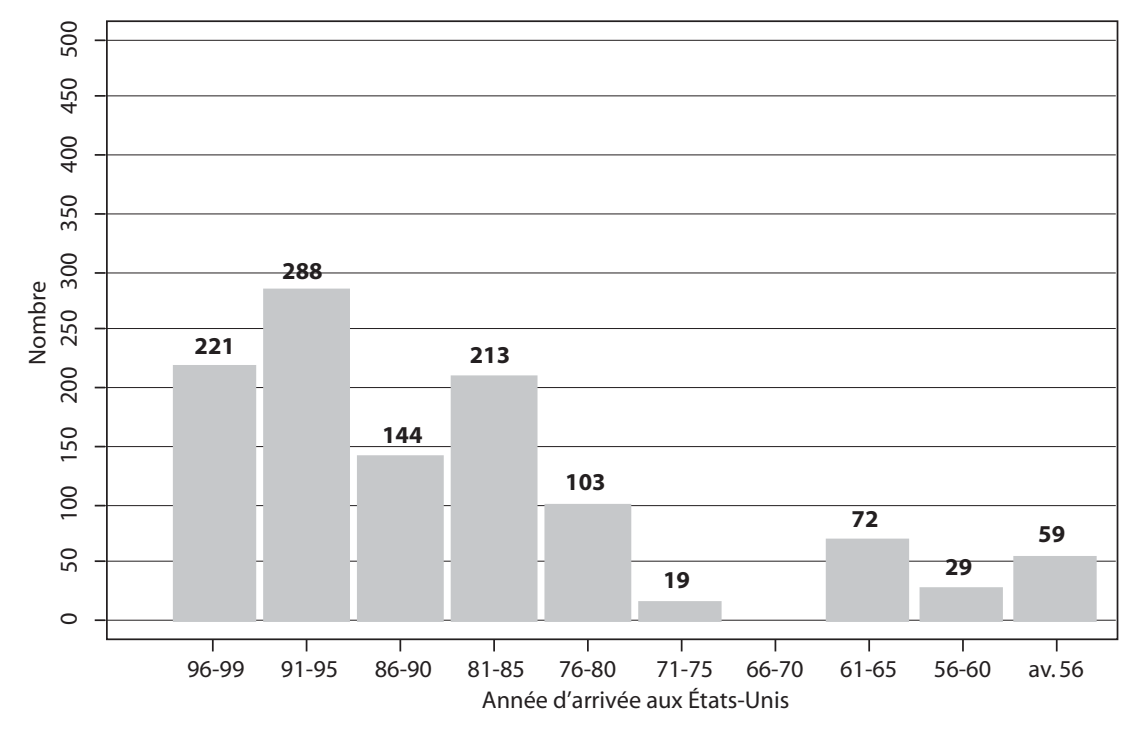


FIGURE 7.2A

Canadiens ayant un doctorat et travaillant aux É.-U.Secteur de l'éducation

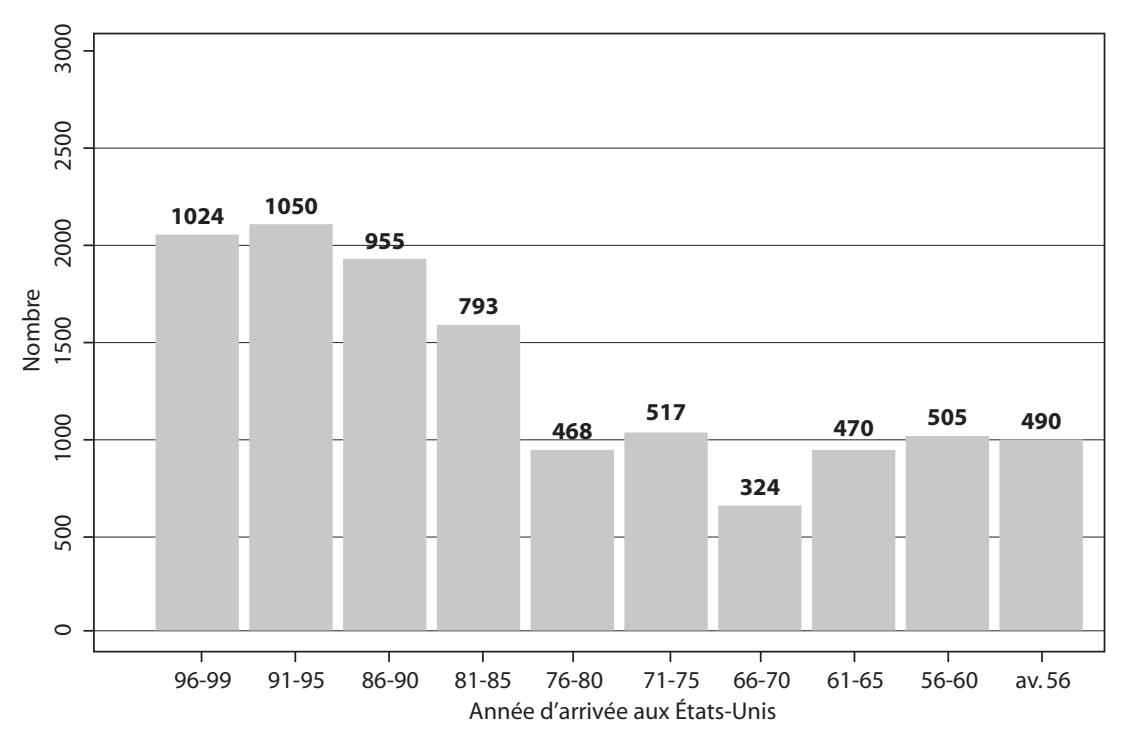

FIGURE 7.2B

Canadiens-français ayant un doctorat et travaillant aux É.-U.Secteur de l'éducation

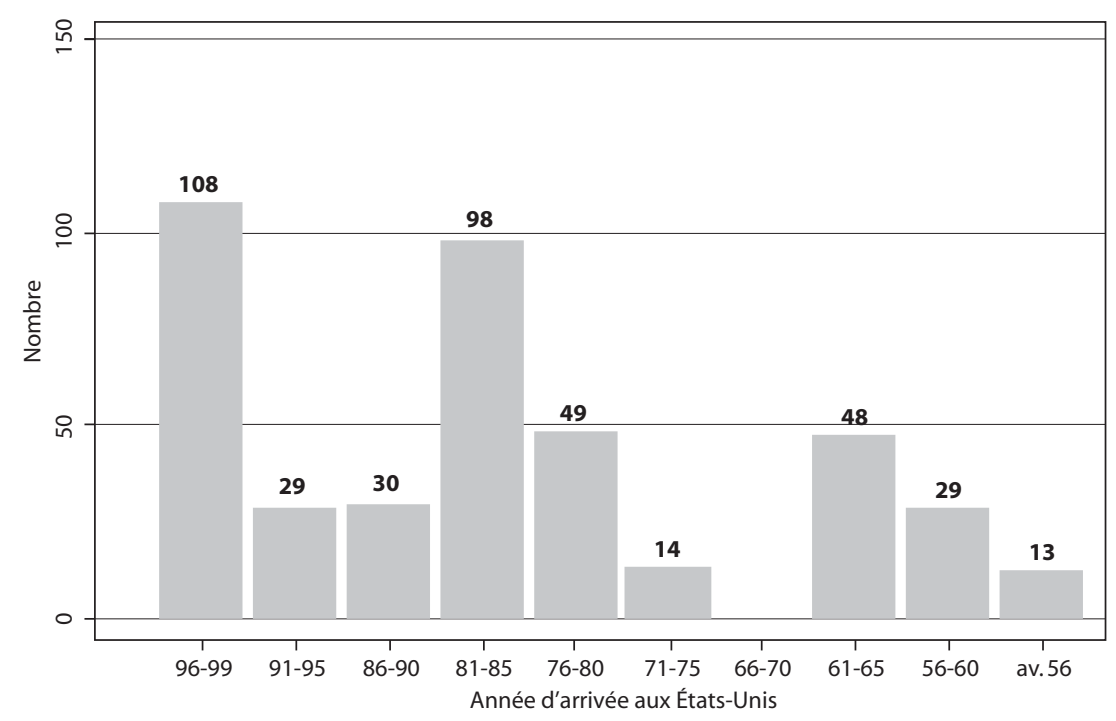


FIGURE 7.3A

Immigrants ayant un doctorat et travaillant au Canada Personnes œuvrant dans tous les secteurs

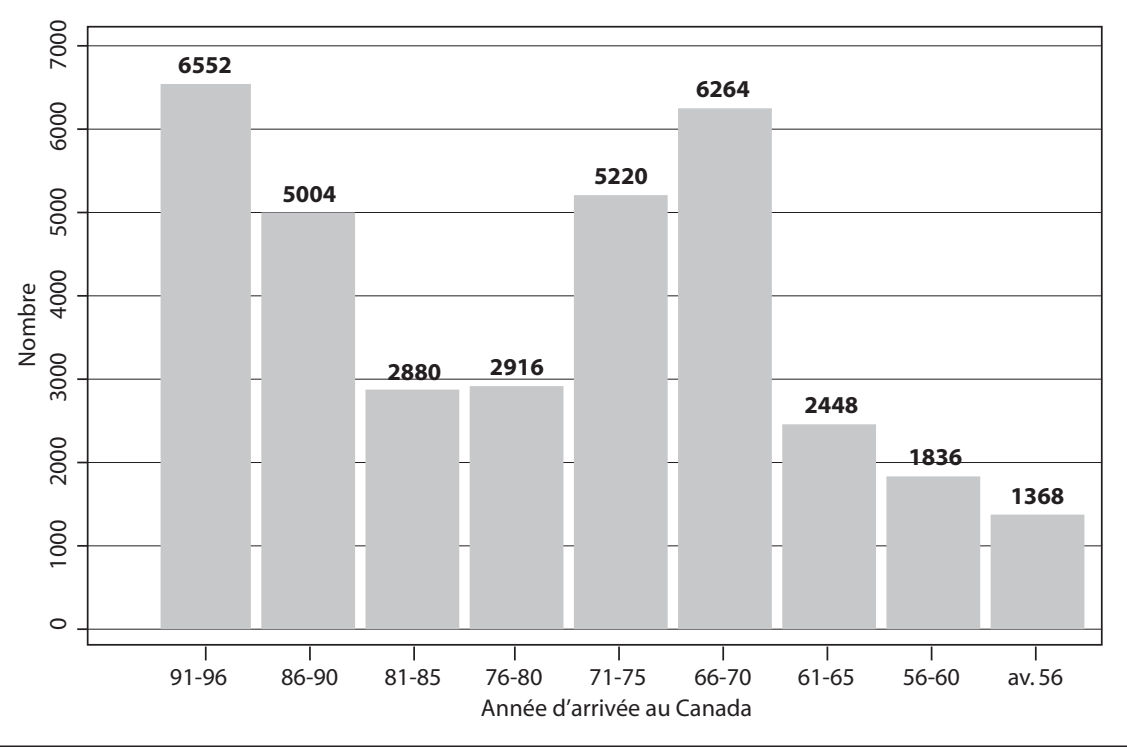

FIGURE 7.3B

Immigrants ayant un doctorat et travaillant au Canada Secteur de l'éducation

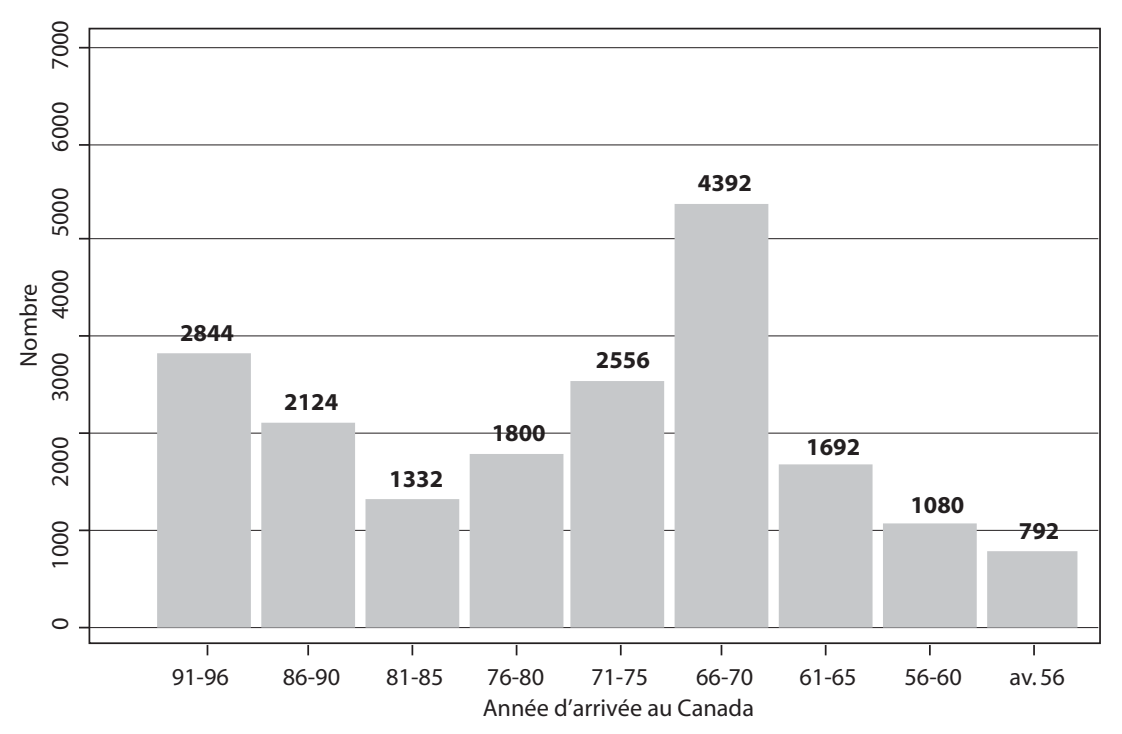


qui déclarent avoir travaillé au cours de l'année précédant l'année de recensement, nous sous-estimons les mouvements migratoires vers les États-Unis observés dans les décennies antérieures aux années 1960, plusieurs de ces émigrants ayant pris leur retraite en l'an $2000^{5}$. Cela étant dit, il apparaît tout de même clair que le nombre d'émigrants s'est accru au cours des dernières années, que ce soit dans tous les secteurs ou dans le seul secteur de l'éducation. Il faut toutefois remarquer que cela, en soi, n'indique en rien l'existence d'un problème qui a tendance à s'aggraver. D'abord, une partie de ces émigrants ont en fait étudié aux États-Unis et ont choisi d'y demeurer à la fin de leurs études. Or, les dernières décennies au Canada ont été caractérisées par une hausse substantielle des taux de fréquentation scolaire à l'université. Il est donc normal que davantage de jeunes Canadiens soient allés faire leurs études doctorales aux États-Unis et il n'est pas impossible que le pourcentage de ceux qui sont revenus ne soit pas très différent de ce qu'il a été historiquement. De plus, comme on peut le voir à la figure 7.3, le nombre d'immigrants détenteurs de doctorats s'est lui aussi accru depuis le début des années 1980. Tout de même, on pourrait argumenter que le ratio du nombre de Canadiens qui émigrent sur le nombre d'immigrants qui entrent s'est détérioré depuis le ratio très faible observé dans les années 1960. Mais même cet argument n'est pas très révélateur en soi. C'est simplement qu'au cours des années 1960 la demande domestique, particulièrement dans le secteur de l'éducation, était tellement forte que beaucoup moins de Canadiens ressentaient le besoin d'aller aux États-Unis pour se trouver un emploi. D’ailleurs, il est intéressant de noter qu'aucun Canadien français résidant aux États-Unis déclare y être arrivé entre 1966 et 1970. C'est justement durant ces années que le système d'éducation québécois a connu un développement inégalé.

Peu importe sous quel angle on examine ces graphiques de flux d'entrées et de sorties, il est clair que beaucoup plus d'immigrants possédant les mêmes qualifications nominales en termes de scolarité complétée sont entrés au Canada qu'il n'y a de Canadiens qui en sont partis. À noter que si l'on examine les flux d'émigration vers les États-Unis au cours des années 1996-2000 en provenance du Canada, donc incluant non seulement les Canadiens de naissance, mais également les immigrants au

5. Les mêmes graphiques obtenus avec les données du recensement américain de 1980 (non inclus dans la présente étude mais disponible auprès des auteurs) montrent un mouvement migratoire important dans les années 1950. 
Canada, le nombre d'entrées surpasse tout de même le nombre de ceux qui ont quitté. Il y a donc peu dans ces graphiques qui laisse deviner la présence d'un problème grave.

Les figures $7.4 \mathrm{a}$ et $7.4 \mathrm{~b}$, quant à elles, montrent la distribution de lâge des Canadiens aux États-Unis. Il est intéressant de noter que ceux-ci ont tendance à être relativement jeunes (trentaine ou jeune quarantaine), les périodes généralement associées à la phase la plus productive dans une carrière scientifique. En revanche, lorsqu'on examine la distribution de l'âge des détenteurs de doctorats au Canada (figure 7.5a), on remarque tout de suite que l'âge médian est passablement plus élevé. À première vue, cela n'est pas très étonnant dans la mesure où l'intensité du phénomène de migration est souvent la plus forte parmi les gens plus jeunes ${ }^{6}$. Il est néanmoins intéressant de constater que le «trou » dans la distribution de lâge au Canada correspond à un sommet dans celle des Canadiens aux États-Unis. De plus, si l'on porte une attention plus particulière à la distribution de l'âge des hommes, on remarque que la distribution de lâge des immigrants établis au Canada (figure 7.5c) est très différente de la distribution de lâge des émigrants canadiens aux États-Unis (figure 7.5b). Cette dernière, tout comme c'est le cas à la figure 7.4a pour les hommes et les femmes réunis, indique clairement qu'une fraction importante des Canadiens établis aux États-Unis se retrouve à un stade du cycle de vie correspondant aux années les plus productives. Cela ne semble pas être le cas pour les immigrants détenteurs de doctorats établis au Canada. De fait, la distribution de l'âge pour ces derniers est assez similaire à la distribution de l'âge des Canadiens récemment émigrés aux États-Unis (figure 7.4b), bien que même cette dernière possède davantage de masse entre 35 et 45 ans que ce n'est le cas pour la distribution des immigrants.

Comme nous l'avons souligné plus tôt, il n'y a aucun indice nous permettant de penser qu'il y ait "pénurie» au sens étroit où les flux migratoires nets de $\mathrm{Ph}$. D. seraient négatifs. Toutefois, si les qualifications nominales sont les mêmes, cela ne signifie pas que les individus soient interchangeables. Les tableaux 7.1, 7.2 et 7.3 illustrent les comparaisons qui nous apparaissent comme étant plus pertinentes. Dans un premier temps, nous quantifions les différences salariales entre les Canadiens

6. Cela est également vrai lorsqu'on examine la «migration» d'un emploi à un autre: la mobilité interemploi est très forte au début de la carrière et diminue avec l'âge. 
FIGURE 7.4A

\section{Distribution de l'âge des émigrants canadiens}

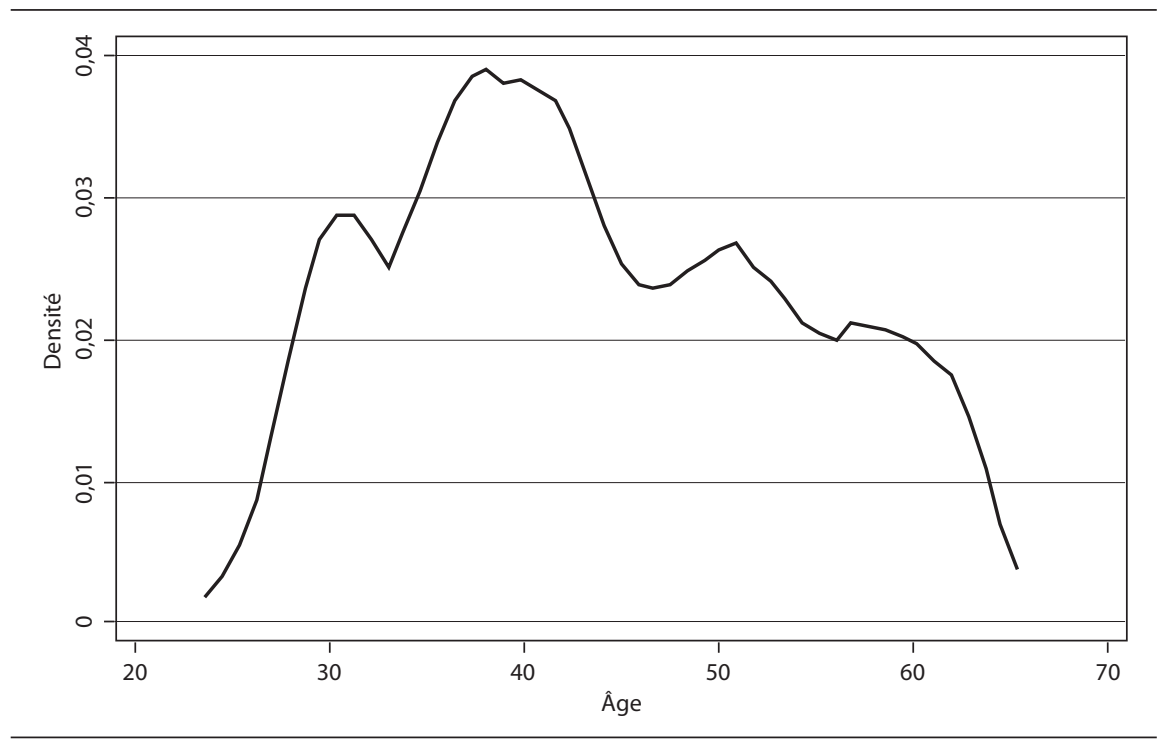

Source: 2000 U.S. Census.

FIGURE 7.4B

\section{Distribution de l'âge des émigrants récents}

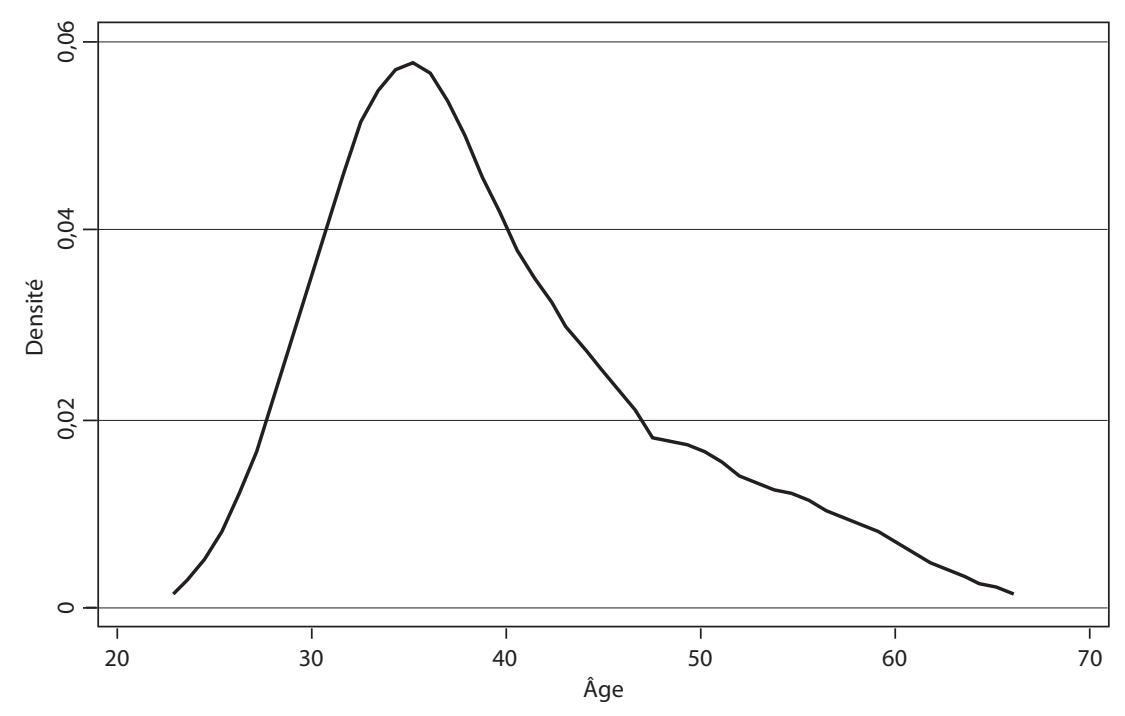

Source: 2000 U.S. Census. 
FIGURE 7.5A

\section{Âge des Canadiens au Canada (hommes)}

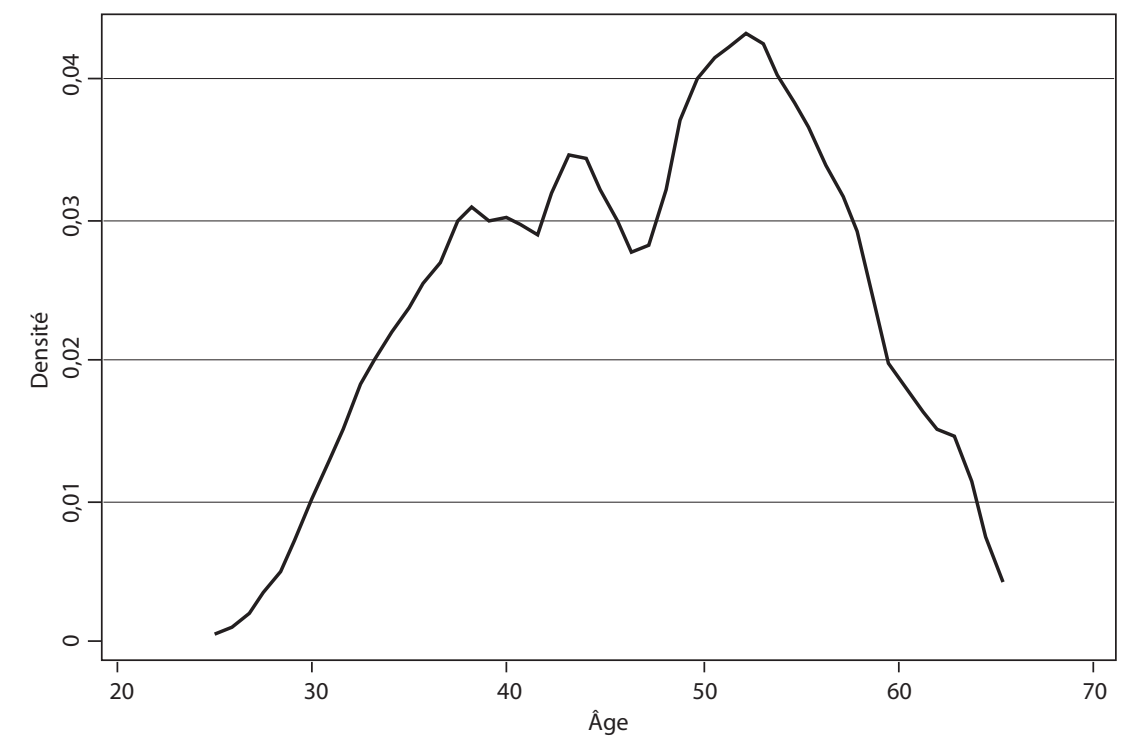

FIGURE 7.5B

Âge des émigrants canadiens aux É.-U. (hommes)

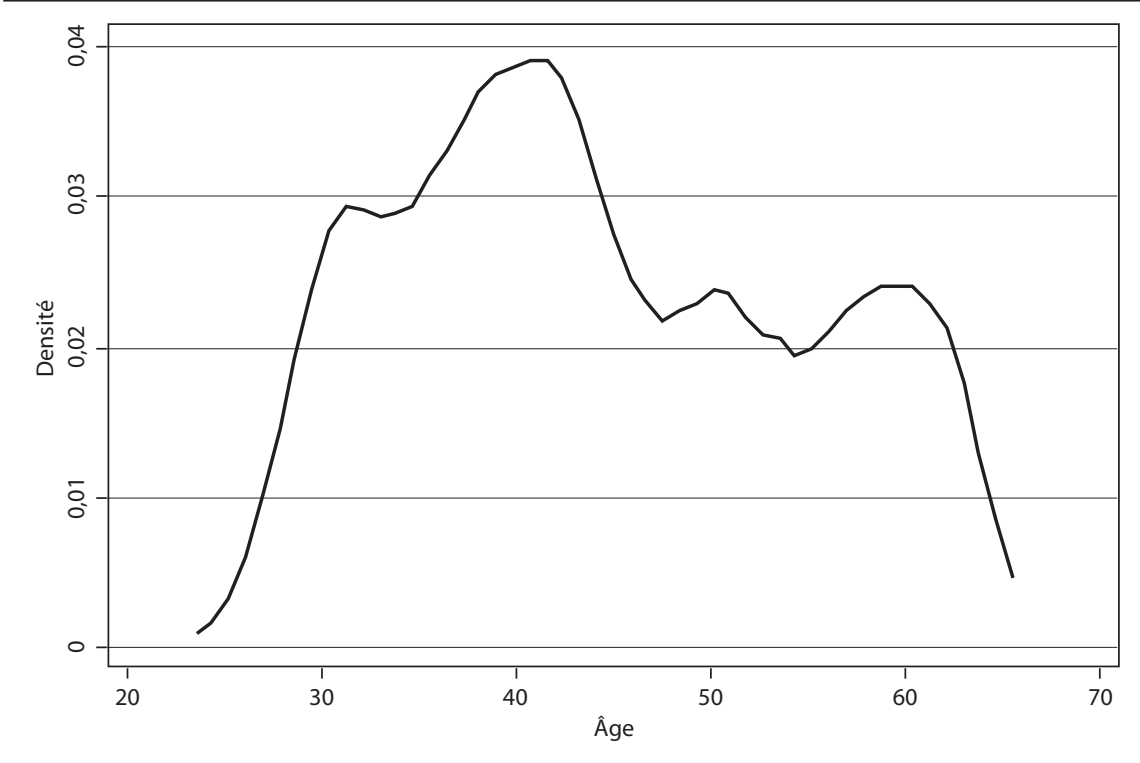


FIGURE 7.5C

\section{Âge des immigrants au Canada (hommes)}

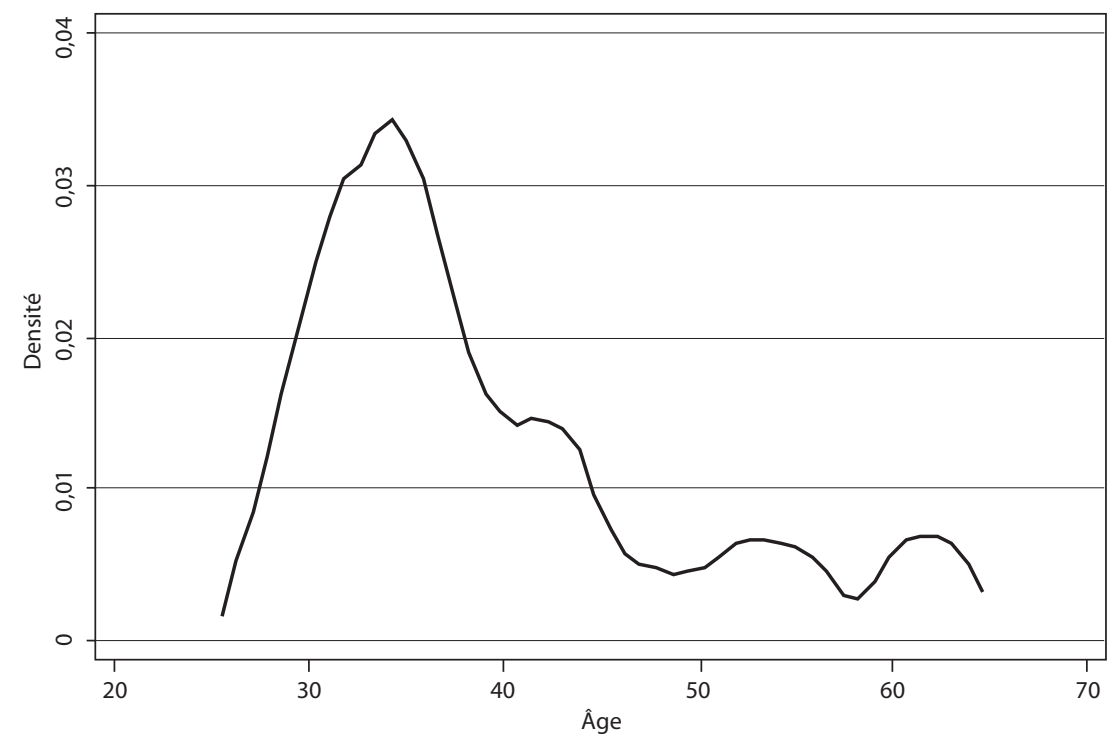

œuvrant aux États-Unis et ceux qui travaillent au Canada. À noter que toutes les comparaisons de salaire tiennent compte des différences dans la structure d'âge des émigrants par rapport aux Canadiens travaillant au Canada ${ }^{7}$.

Il ressort clairement du tableau 7.1 que les années 1990 ont vu les pressions à la hausse exercées sur les salaires aux États-Unis augmenter considérablement, que ce soit pour toutes les cohortes confondues ou seulement la cohorte entrée aux États-Unis entre 1996 et 1999. Avec de tels écarts, il n'est pas étonnant que certains secteurs aient connu des difficultés sur le plan de la rétention. En ce qui concerne le salaire des Canadiens aux États-Unis par rapport à celui des Américains de naissance (tableau 7.2), il est intéressant de noter que tandis que les salaires américains ont connu une nette progression à partir des années 1980,

7. Nous estimons la différence en log du salaire des Canadiens aux États-Unis par rapport aux Canadiens au Canada par une régression linéaire du log du salaire hebdomadaire sur une variable dichotomique indiquant le statut de résidence aux États-Unis en plus d'une série de variables dichotomiques indiquant l'âge des répondants. Donc, les résultats montrés dans les tableaux font référence à la différence du log du salaire, qui est approximativement égale à la différence en pourcentage. 
les salaires relatifs des Canadiens ont chuté, à tout le moins lorsque l'on compare les salaires en 2000 avec ceux en 1980. Au fond, cela n'est guère étonnant. Il y a 20-25 ans, les conditions salariales dans les universités canadiennes (pour ne mentionner que ce secteur) étaient similaires à celles offertes dans les universités américaines. Par conséquent, une université américaine qui voulait attirer un Canadien devait payer une prime substantielle de façon à rendre la perspective d'une migration suffisamment attirante. Aujourd'hui, les universités américaines n'ont plus besoin de faire cela: tout ce qu'elles ont à faire est d'offrir une prime suffisamment supérieure à ce que paient les universités canadiennes, et comme ces dernières paient passablement moins qu'aux États-Unis, il en découle une détérioration relative pour les émigrants, surtout les plus récents. Naturellement, ces derniers pourront voir leurs salaires aux États-Unis ajustés à la hausse dans les années suivant leur arrivée.

Finalement, nous voyons au tableau 7.3 la différence salariale au Canada entre les Canadiens de naissance et les immigrants. En gros, les deux groupes de détenteurs de doctorats, toutes cohortes d'immigrants confondues, gagnent des salaires semblables. Ce que cela indique, c'est que la «qualité» des deux groupes est similaire. Naturellement, contrairement aux États-Unis, la distribution des salaires tend à être plus égalitaire, ce qui signifie qu'a priori on ne s'attendrait pas à voir des différences moyennes très grandes de toute façon. Toutefois, ce que cela illustre aussi, c'est que les meilleurs parmi les immigrants fortement scolarisés sont susceptibles de ne pas choisir le Canada et de choisir plutôt les États-Unis, et ce, pour les mêmes raisons qui amènent certains des Canadiens les plus talentueux à émigrer vers les États-Unis. Ce qui est peut-être plus inquiétant, c'est que la cohorte la plus récente d'immigrants est beaucoup plus défavorisée par rapport aux Canadiens que lors des recensements précédents. Étant donné les compressions effectuées dans le milieu universitaire au milieu des années 1990, un peu partout au Canada, cela n'est peut-être pas tout à fait étonnant: les perspectives d'emploi étaient tout simplement moins bonnes à ce moment-là et les récentes cohortes ont sans doute eu plus de difficultés qu'auparavant à se trouver un emploi correspondant à leurs qualifications. 
TABLEAU 7.1

\section{Salaire des Canadiens de naissance ayant un doctorat et travaillant aux États-Unis comparé au salaire des Canadiens titulaires de doctorat au Canada}

\begin{tabular}{|l|c|c|c|c|}
\hline \multirow{2}{*}{ Année de recensement } & \multicolumn{2}{|c|}{ Tous les secteurs } & \multicolumn{2}{c|}{ Secteurs de l'éducation } \\
\cline { 2 - 5 } & Tous les individus & $\begin{array}{c}\text { Travailleurs à plein } \\
\text { temps seulement }\end{array}$ & Tous les individus & $\begin{array}{c}\text { Travailleurs à plein } \\
\text { temps seulement }\end{array}$ \\
\hline Partie A: Émigrants canadiens de toutes les cohortes \\
\hline 1980-1981 & 13,9 & 20,6 & 1,0 & 5,2 \\
\hline 1990-1991 & 2,0 & 2,7 & $-6,6$ & $-4,1$ \\
\hline 2000-1996 & 45,0 & 44,6 & 30,6 & 30,1 \\
\hline Partie B: Canadiens ayant émigré aux É.-U. au cours des cinq années précédant chaque recensement \\
\hline 1980-1981 & 19,2 & 25,6 & 10,6 & 16,7 \\
\hline 1990-1991 & $-13,8$ & $-6,8$ & $-11,0$ & $-1,8$ \\
\hline 2000-1996 & 34,4 & 22,4 & 28,2 & 17,8 \\
\hline
\end{tabular}

Note: Le contenu des cellules représente la différence du log du salaire hebdomadaire, ajusté en fonction de l'âge, du taux de change et de l'inflation. La première année de chaque paire représente l'année de recensement aux États-Unis alors que la deuxième nous donne l'année de recensement au Canada.

TABLEAU 7.2

\section{Salaire des Canadiens de naissance ayant un doctorat et travaillant aux États-Unis comparé au salaire des Américains détenteurs de doctorat aux É.-U.}

\begin{tabular}{|l|c|c|c|c|}
\hline \multirow{2}{*}{ Année de recensement } & \multicolumn{2}{|c|}{ Tous les secteurs } & \multicolumn{2}{c|}{ Secteurs de l'éducation } \\
\cline { 2 - 5 } & Tous les individus & $\begin{array}{c}\text { Travailleurs à plein } \\
\text { temps seulement }\end{array}$ & Tous les individus & $\begin{array}{c}\text { Travailleurs à plein } \\
\text { temps seulement }\end{array}$ \\
\hline Partie A: Émigrants canadiens de toutes les cohortes \\
\hline 1980-1981 & 11,8 & 15,5 & 5,1 & 9,7 \\
\hline 1990-1991 & 0,1 & $-3,2$ & $-3,8$ & $-1,5$ \\
\hline 2000-1996 & 6,4 & 5,5 & 3,6 & 4,8 \\
\hline Partie B: Canadiens ayant émigré aux É.-U. au cours des cinq années précédant chaque recensement \\
\hline 1980-1981 & 12,3 & 15,5 & 5,1 & 16,7 \\
\hline 1990-1991 & $-3,5$ & $-7,0$ & $-12,1$ & $-14,6$ \\
\hline 2000-1996 & 1,9 & 10,3 & $-6,6$ & $-14,6$ \\
\hline
\end{tabular}

Note: Le contenu des cellules représente la différence du log du salaire hebdomadaire, ajusté en fonction de l'âge. 
TABLEAU 7.3

\section{Salaire des Canadiens de naissance ayant un doctorat et travaillant au Canada comparé au salaire des immigrants détenteurs de doctorat au Canada}

\begin{tabular}{|l|c|c|c|c|}
\hline \multirow{2}{*}{ Année de recensement } & \multicolumn{3}{|c|}{ Tous les secteurs } & \multicolumn{2}{c|}{ Secteurs de l'éducation } \\
\cline { 2 - 5 } & Tous les individus & $\begin{array}{c}\text { Travailleurs à plein } \\
\text { temps seulement }\end{array}$ & Tous les individus & $\begin{array}{c}\text { Travailleurs à plein } \\
\text { temps seulement }\end{array}$ \\
\hline Partie A: Immigrants de toutes les cohortes & & $-0,1$ & $-1,3$ \\
\hline 1981 & 3,1 & 1,6 & 2,3 & $-0,5$ \\
\hline 1991 & $-1,6$ & $-3,0$ & $-0,6$ & $-0,7$ \\
\hline 1996 & 3,3 & 3,1 & 21,3 & 10,7 \\
\hline Partie B: Immigrants arrivés au Canada, au cours des cinq années précédant chaque recensement \\
\hline 1981 & 9,2 & 5,1 & 8,7 & 1,5 \\
\hline 1991 & 8,2 & 2,2 & 21,6 & 13,0 \\
\hline 1996 & 34,9 & 26,8 & \\
\hline
\end{tabular}

Note: Le contenu des cellules représente la différence du log du salaire hebdomadaire, ajusté en fonction de l'âge.

\section{DISCUSSION}

Évidemment, le salaire n'est pas tout dans la vie; les conditions de vie dans le pays d'accueil comptent également. De plus, les comparaisons de salaires décrites ci-dessus ne tiennent pas compte des champs de spécialisation: les différences seraient plus faibles s'il se trouvait que les émigrants qui quittent pour les États-Unis œuvrent dans des secteurs particulièrement payants alors que les Canadiens qui restent travaillent par exemple dans le secteur des lettres et sciences humaines. Enfin, les conditions non salariales jouent certainement aussi un rôle important. On peut penser à la décision d'émigrer ou non comme étant influencée par une variété de facteurs et il n’est pas nécessairement clair qu'à la marge il ne faille modifier que le salaire pour retenir les meilleurs talents. Toutefois, des écarts de salaires tels que ceux mentionnés montrent bien, à notre avis, qu'il faut prendre avec un gros grain de sel les simples comparaisons de flux d'entrées et de sorties. Ce qui compte lorsqu'on parle de fuite des cerveaux, ce n'est pas en soi de perdre les talents «moyens» (si l'on en vient à s'inquiéter de cela, c'est alors que le problème est encore plus grave qu'il ne l'est à l'heure actuelle!), c'est bien plutôt le départ de ceux qui sont meilleurs que la moyenne. Ce sont précisément eux qui sont convoités par les universités et centres de recherche américains. 
Nous sommes amenés à penser que le phénomène ne se résorbera pas de sitôt, car il résulte en partie d'un contexte strictement américain sur lequel nous n'avons aucun contrôle, soit l'expansion très substantielle des inégalités aux États-Unis depuis le début des années 1980 (voir, par exemple, DiNardo, Fortin et Lemieux, 1996, et Katz et Murphy, $1992^{8}$ ). Une des principales composantes de cet accroissement des inégalités est la croissance de l'avantage salarial des plus scolarisés par rapport aux moins scolarisés. Cela est vrai pour les détenteurs d'un baccalauréat par rapport aux diplômés du secondaire et ce l'est encore plus pour les vedettes détentrices d'un doctorat. Bien que cette croissance des inégalités se soit plus ou moins arrêtée depuis à peu près la fin des années 1990, sinon avant, il en résulte que les salaires payés par les universités américaines combinés avec des conditions de travail très attrayantes (charge d'enseignement réduite, fonds de recherche, etc.) font qu'il est plus difficile que jamais de rendre le Canada attrayant pour les plus talentueux œuvrant dans certains secteurs bien définis. Le fameux giant sucking sound va continuer de se faire entendre à moins que des mesures ne soient prises, que ce soit sous la forme de salaires qui s'ajustent de façon plus flexible afin de récompenser/attirer les meilleurs éléments ou par la création d'un environnement de recherche plus stimulant, par exemple par la réduction de la charge d'enseignement dans les universités de recherche. Comme illustré au tableau 7.1, les données les plus récentes montrent assez clairement que l'écart salarial entre les États-Unis et le Canada, soit les salaires des Canadiens aux États-Unis vs les salaires versés au Canada, s'est considérablement accru au cours des années 1990. Ce qui est peut-être encore plus troublant, c'est que les émigrés récents ont vraisemblablement contribué de façon importante au creusement de cet écart (tableau 7.1, partie B). Comme on l'a vu à la figure 7.4b, les récents émigrants vers les États-Unis sont recrutés en grande partie parmi les éléments relativement jeunes (mais expérimentés, ceux qui en quelque sorte atteignent ou sont sur le point d'atteindre les années les plus productives de leur carrière).

Mais jusqu'à quel point doit-on tenter de concurrencer les riches universités américaines? À notre avis, il est illusoire de penser pouvoir offrir les mêmes conditions qu'aux États-Unis: après tout, beaucoup de chercheurs ambitieux préféreront toujours, à salaire égal, être à l'emploi des Harvard et Princeton de ce monde. Par conséquent, si l'on s'engage

8. Même s'il y a également eu une hausse des inégalités au Canada (Morissette, 1995), elle a été de moindre ampleur qu'aux États-Unis. 
dans une surenchère afin d'acquérir les services de gens qui n'ont aucune raison particulière de vouloir vivre ici, ceux-ci quitteront de toute façon lorsque la bonne offre arrivera. En réalité, ceux qui doivent être visés sont ceux qui manifestent une attirance pour le Canada et le Québec, mais qui ne voient pas comment ils pourraient venir y travailler étant donné les différences substantielles dans les conditions globales de travail, qu'elles touchent le salaire ou la charge d'enseignement comme en ce moment. À cet égard, des interventions plus ciblées, comme les chaires de recherche du Canada ou la Fondation canadienne pour l'innovation qui finance les infrastructures de recherche, représentent probablement une meilleure façon de s'attaquer au problème en comparaison d'une intervention plus globale qui ferait augmenter les salaires de tout le monde, même ceux qui n'ont aucune volonté d'émigrer et/ou qui ne sont pas attirés par les universités ou firmes américaines. Le même raisonnement vaut pour la répartition des charges d'enseignement. Réduire la charge d'enseignement de tout le monde afin de retenir ou d'attirer les meilleurs chercheurs représenterait une stratégie très coûteuse par rapport aux bénéfices potentiels qu'on en tirerait.

\section{BIBLIOGRAPHIE}

CARD, David (2003). « Canadian Emigrants in the United States », ronéo, Department of Economics, Berkeley.

DiNardo, John (1999). Nicole Fortin et Thomas Lemieux (1996). «Labor Market Institutions and the Distribution of Wages, 1973-1992: A SemiParametric Approach », Econometrica, 64(5), septembre, p. 1001-1044.

Helliwell, John (1999). "Checking the Brain Drain: Evidence and Implications», Policy Options, Montréal, Institut de recherche en politique publique (IRPP).

Katz, Lawrence F. et Kevin Murphy (1992). "Changes in Relative Wages, 1963-1987: Supply and Demand Factors », Quarterly Journal of Economic, 107(1), février, p. 35-78.

MorissetTe, René (1995). «Why Has Inequality in Weekly Earnings Increased in Canada?», Statistics Canada Analytical Studies Branch Research paper $\mathrm{n}^{\circ} 80$.

Zhao, John, Doug Drew et T. Scott Murray (2000). «Brain Drain and Brain Gain: The Migration of Knowledge Workers from and to Canada », Education Quarterly Review, 6(3), Ottawa, Statistics Canada, Catalogue 81-003. 

Deuxième partie

L'INSERTION SOCIOPROFESSIONNELLE Le point de vue du Conseil national des cycles supérieurs 



\section{Chapitre}

\section{L'INSERTION, UN CONCEPT EN REDÉFINITION}

D’après plusieurs études menées sur les diplômés, le meilleur indicateur retenu pour mesurer l'insertion est celui de la stabilité d'emploi. Selon la définition proposée par Vincens ${ }^{1}$, l'insertion (dans sa définition la plus traditionnelle) est complétée et réussie lorsque le diplômé occupe un emploi qu'il ne prévoit pas quitter ou perdre à court terme et qu'il considère que cet emploi lui permet de réaliser son projet de vie.

Les nombreuses Relance effectuées auprès des diplômés nous renseignent également sur le degré de concordance existant entre la formation initiale du diplômé et l'emploi qu'il a obtenu. Cet indicateur a pour assise le postulat traditionnel de l'adéquation formation-emploi qui sous-entend qu'on doit planifier la formation en fonction des besoins du marché en considérant qu'à une catégorie d'emploi donnée correspond un type de formation précis.

Les données issues des enquêtes sur l'insertion des diplômés nous renseignent en somme sur la situation de ces derniers une fois qu'ils ont intégré le marché de l'emploi. Ces renseignements sont en quelque

1. J. Vincens, «L'insertion dans la vie active», dans L'insertion professionnelle des jeunes à la sortie des études postsecondaires, dossier $n^{\circ} 2$, Université catholique de Louvain, Institut des sciences du travail, Louvain, 1981. 
sorte limités à la perception traditionnelle de l'insertion. De fait, la façon dont on mesure l'insertion est basée sur des concepts qui ont peu évolué depuis 20 ans. Or le marché du travail, lui, a évolué significativement, ce qui fait en sorte que les préceptes d'une insertion réussie tels qu'ils ont été élaborés au début des années 1980 ne peuvent plus être appliqués aux réalités actuelles de l'insertion professionnelle. Dans le présent chapitre, nous nous basons grandement sur les travaux de Trottier pour questionner le concept classique d'insertion. D'abord, nous remettons en perspective les idées relatives à la stabilité d'emploi et au lien formationemploi, considérant à ce sujet les attentes et les perceptions des diplômés. Puis, nous prenons soin d'analyser le processus d'insertion, ainsi que la manière dont le diplômé y contribue.

\section{MOUVANCE ET INSTABILITÉ DU MARCHÉ DE L'EMPLOI: MISER SUR L'EMPLOYABILITÉ DE L'INDIVIDU}

On constate aisément que les emplois sont moins stables qu'auparavant, c'est-à-dire que le marché du travail est en évolution perpétuelle; on a grand besoin de gens qualifiés, mais la nature des besoins change souvent. Les entreprises sont instables, constamment en concurrence avec des joueurs de partout. Selon le développement des technologies, l'évolution des marchés et la réorganisation des entreprises, la demande varie significativement dans le temps. Dans un tel contexte, comment arrimer les intérêts des diplômés et ceux du marché du travail?

Il est vrai qu'une grande proportion de jeunes vivent une situation d'emploi caractérisée par le travail temporaire, autonome ou à temps partiel et, conséquemment, par la précarité. Il faut souhaiter qu'on intervienne socialement et politiquement pour atténuer cette précarité, mais ses causes étant structurelles, la situation risque fort de perdurer. Toutefois, il faut se rappeler que les diplômés n'évoluent pas hors contexte et qu'ils sont familiers avec cette caractéristique du marché du travail. Ainsi, selon les observations réalisées par Trottier, les jeunes, et parmi eux les diplômés de cycles supérieurs, ont remis en perspective les indicateurs de stabilité traditionnellement utilisés pour mesurer l'insertion. Comme le décrit l'auteur, «[...] tout se passe comme s'ils assumaient cette précarité et avaient intériorisé les conditions objectives qui rendent 
peu probable qu'ils obtiennent dès leur entrée sur le marché du travail un emploi à durée indéterminée ${ }^{2} »$. On peut donc affirmer que les attentes des diplômés se sont modelées à la réalité du travail d'aujourd'hui.

Le marché du travail a changé, la nature des emplois qu'on y retrouve aussi, et, corrélativement, la relève en main-d'œuvre évolue de façon à assurer son insertion et son intégration à l'intérieur des paramètres actuels. Il importe alors de revoir, dans un marché du travail où l'instabilité et la mouvance sont devenues les règles, la façon dont la précarité vécue par les diplômés influence notre idée de leur insertion. Doit-on considérer que le fait d'effectuer des contrats à durée déterminée signifie que l'insertion n'est pas réussie? Doit-on conclure que le travail autonome n'est pas une option menant à l'insertion professionnelle?

Trottier propose de troquer la traditionnelle vision de la stabilité d'emploi pour celle d'une stabilité globale sur le marché du travail.

Tout se passe comme si la stabilisation sur le marché du travail était beaucoup moins qu'auparavant liée à la permanence de l'emploi et du poste qu'un individu occupe, mais davantage à l'employabilité de l'individu, à sa capacité d'éviter le chômage, de mettre en valeur ses compétences et sa polyvalence, soit en démontrant que ses compétences correspondent à celles qu'un employeur éventuel recherche, soit en créant son propre emploi ${ }^{3}$.

Ainsi, plusieurs jeunes arrivent à répondre au besoin de flexibilité des entreprises et à se maintenir relativement stables sur le marché du travail, même si les employeurs, eux, sont plus instables. Dans un contexte de «volatilité» de l'emploi, c'est l'employabilité de l'individu qui est la clé de son insertion, ainsi que sa capacité à répondre aux besoins des employeurs dans les secteurs d'activité où il gravite. Cela exige des diplômés une grande flexibilité et une remise en question des principes habituels de l'emploi stable et de la précarité. Aujourd'hui, des gens peuvent évoluer dans cette mouvance tout au long de leur carrière sans pour autant se considérer en situation "précaire».

2. C. Trottier, «Le rapport au travail et l'accès à un emploi stable, à temps plein, lié à la formation. Vers l'émergence de nouvelles normes?», dans Les 18 à 30 ans et le marché du travail - Quand la marge devient la norme, 2000, Québec, Presses de l'Université Laval, p. 47.

3. Ibid., p. 46-47. 
L'instabilité offre d'ailleurs à certains jeunes le prétexte pour créer leur propre entreprise, afin de répondre aux nouveaux besoins sans se limiter à la réalisation de divers petits contrats pour plusieurs compagnies. Ces jeunes développent ainsi une expertise qu'ils peuvent par la suite offrir aux divers employeurs. La multiplication du travail autonome et de l'entrepreneuriat est, de fait, une tendance forte ces dernières années.

Les données montrent toutefois une dégradation importante des conditions d'emploi et de vie pour les employés à temps partiel, temporaires et autonomes. Ainsi, la législation et les programmes sociaux doivent être adaptés en conséquence ${ }^{4}$. Les travailleurs précaires sont mal protégés, c'est vrai, mais ce qui ajoute à leurs difficultés, c'est qu'ils sont souvent mal outillés pour s'aventurer sur un marché de l'emploi où le développement de l'employabilité est essentiel.

Actuellement, les données sur l'insertion se basent sur une vision de cheminement par étapes. Pour répondre à des besoins de prévision économique, d'orientation ou de planification basés sur des analyses macroéconomiques, la vision de l'insertion est rectiligne. Il y a la formation, puis le début de l'insertion, puis la fin de l'insertion... Il s'agit d'une simplification de la réalité qui peut difficilement servir de base à nos objectifs. Aujourd'hui, l'insertion ne peut être si facilement circonscrite. L'insertion constitue rarement un état final. Une fois qu'il aura réussi une première insertion, le diplômé sera probablement appelé à poursuivre le processus à différents moments au cours de sa vie active.

Parallèlement à tout cela, d'autres aspects de la vie des diplômés doivent être pris en considération. Il faut concilier les désirs d'avoir des enfants, de fonder une famille, d'acquérir une propriété et de s'établir au sein d'une communauté. Certains recherchent, notamment pour ces raisons, des emplois stables. D'autres, selon les priorités et les caractères, ne seront pas nécessairement intéressés par la stabilité d'emploi, mais par la possibilité d'avoir un ou des emplois valorisants. Ils évolueront très bien dans le contexte de mouvance et miseront davantage sur leur employabilité.

4. Conseil permanent de la jeunesse, Emploi atypique et précarité chez les jeunes: une maind'œuvre à bas prix, compétente et jetable, op. cit., note 19, 114 p. 
Sur le plan de la formation, les périodes de précarité vont souvent permettre aux jeunes de réfléchir sur ce qu'ils veulent vraiment faire, d'acquérir une formation complémentaire, de mettre à l'épreuve leurs compétences et leurs objectifs de carrière afin de mieux définir ce qui leur convient. Comme société, il importe bien entendu de leur apporter un soutien efficace et structuré. En somme, l'approche de la stabilisation en emploi par l'employabilité repose sur le développement par les diplômés de leur potentiel professionnel et personnel. Elle permet de miser sur l'individu comme acteur de sa propre insertion, mais impose de lui donner les outils pour faciliter son adaptation aux nouvelles réalités.

\section{REPRÉSENTATIONS ET ATTENTES DES DIPLÔMÉS FACE À L'INSERTION}

Le CNCS-FEUQ croit qu'il est primordial, dans une démarche où l'on cherche à définir et à circonscrire l'insertion des diplômés, de considérer leurs perceptions et leurs attentes à l'égard du processus d'insertion. Nous relevions précédemment que les jeunes, et plus précisément les diplômés de cycles supérieurs, ont à composer avec une réalité du travail qui leur demande sans cesse de s'adapter, de réévaluer et de revoir leurs objectifs d'insertion. En d'autres mots, ils doivent se demander si la réalité qui se présente à eux répond à leurs attentes, et si elle est conciliable avec les autres aspects de leur vie.

Devant cette remise en question, les réactions des diplômés peuvent être diverses. Or, pour bien juger de l'état de l'insertion, il faut chercher à connaître ces réactions. Aux yeux des diplômés, est-ce que la vie professionnelle perd de l'intérêt en raison de la précarité vécue? Sont-ils désillusionnés? Essaient-ils de prendre une distance par rapport à tout cela? À quelle proportion de diplômés un contexte de mouvance convient-il? Combien recherchent à tout prix des emplois stables?

Afin de trouver réponses à ces questions, Trottier reprend les résultats de deux enquêtes menées en Allemagne et au Québec ${ }^{5}$ sur le rapport des jeunes au travail. Il souligne que pour ces derniers, «[...] l'objectif de se trouver du travail, de s'insérer dans la vie professionnelle pour

5. M. Beathe, «Le rapport au travail des jeunes», dans Jeunesses et sociétés - Perspectives de la recherche en France et en Allemagne, sous la direction de G. Mauger, R. Bendit et C. Wolfferdorff, Paris, Armand Colin, 1994, p. 151-166.

G. Fournier et L. Croteau, «Perceptions et attentes vis-à-vis du travail des jeunes diplômés », dans Psychologie du travail et des organisations, vol. 3, $\mathrm{n}^{\text {os }} 3-4,1998$, p. 89-108. 
gagner leur vie demeure primordial. Loin d'avoir diminué l'importance accordée au travail, la crise de l'emploi chez les jeunes a plutôt renforcé leurs valeurs à l'égard du travail ${ }^{6}$.»

En résumé, la très grande majorité des diplômés ont une conception expressive du travail. Cela signifie que, pour eux, le travail fait partie intégrante du projet de vie et il doit leur permettre de se réaliser, de s'épanouir et de se développer. Ainsi, il est indéniable que les diplômés ont des attentes face à leur entrée sur le marché du travail et à leur cheminement professionnel. Les approches traditionnellement retenues pour mesurer l'insertion sont de nature plus quantitative alors que l'approche qui consiste à tenir compte des représentations et des attentes que les diplômés ont au regard de leur insertion est de nature plus qualitative ${ }^{7}$. Cette méthode permet non seulement d'avoir un portrait de la situation des diplômés sur le marché du travail, mais également de qualifier cette insertion et d'en définir les variables déterminantes.

Afin de cerner les représentations d'une insertion réussie chez les diplômés universitaires, Trottier et son équipe ont procédé à des entrevues semi-dirigées auprès de 61 bacheliers. De cette démarche, six thèmes ont été dégagés comme faisant partie intégrante de la perception des diplômés à l'égard du processus d'insertion ${ }^{8}$. Nous présentons ci-dessous les grandes lignes évoquées pour chacun des thèmes.

\section{La stabilité d'emploi}

- Pour environ le tiers des diplômés interrogés, le thème de la stabilité d'emploi continue d'être important à leurs yeux. Ces diplômés évoquent la difficulté des situations précaires et estiment qu'une stabilité d'emploi est plus sécurisante.

6. C. Trottier, «Le rapport au travail et l'accès à un emploi stable, à temps plein, lié à la formation. Vers l'émergence de nouvelles normes?», op. cit. note 39, p. 38.

7. C. Trottier, L. Laforce et R. Cloutier, «Les représentations de l'insertion professionnelle chez les diplômés de l'université », dans Formation Emploi, n 58, Centre d'études et de recherches sur les qualifications (Céreq), Marseille, 1997, p. 61-77.

8. Ibid., p. 63-72. 


\section{L'employabilité individuelle}

- Près de la moitié des diplômés considère que c'est l'employabilité de chacun qui assure aujourd'hui sa stabilité sur le marché du travail. «On ne peut plus [selon eux], dans la conjoncture actuelle, mesurer la stabilité d'emploi uniquement par le fait de détenir un emploi à durée indéterminée $(\mathrm{CDI})^{9}$.»

\section{La participation au marché du travail}

- Pour $10 \%$ des diplômés interrogés, l'insertion est assimilée à la simple participation au marché du travail. Selon cette perception, être inséré signifie simplement avoir un emploi dont la fonction est principalement d'assurer un revenu décent.

\section{La correspondance formation / emploi}

- Plus de la moitié des diplômés interrogés ont précisé que la stabilité d'emploi (ou la stabilité sur le marché du travail) ne suffit pas à elle seule à assurer une insertion réussie. Il faut également que l'emploi occupé corresponde à la formation reçue. Il est important de noter que les diplômés qui valorise le lien entre la formation et l'emploi ont critiqué la formation qu'ils ont reçue, car celle-ci ne leur avait pas apporté le bagage «pratique» nécessaire pour se préparer et répondre aux exigences du marché du travail ${ }^{10}$.

- L'importance que revêt les diplômés pour la concordance entre l'emploi occupé et la formation reçue varie d'une personne à l'autre. Pour certains, ce lien est primordial et doit être très fort alors que, pour d'autres, l'utilisation des compétences développées au cours des études (par exemple, l'esprit critique ou la capacité d'analyse) sera satisfaisante. L'importance de cet élément pour les diplômés varie notamment en fonction du type de formation reçue, du secteur d'étude, de la conjoncture économique, du cheminement ou du changement d'orientation professionnelle.

9. Ibid., p. 64 .

10. Ibid., p. 67-68. 


\section{L'engagement dans le travail}

- Près d'un étudiant sur cinq signale qu'il est important pour lui d'occuper un travail stimulant qui fait appel à ses habiletés et à ses compétences professionnelles. Les diplômés voient leur emploi comme un engagement, un moyen de se dépasser et de se réaliser.

\section{L'intégration dans le milieu du travail et la profession}

- Les diplômés (près de la moitié) recherchent également un travail qui leur permettra de s'intégrer à des réseaux professionnels et qui leur assurera la reconnaissance de leurs pairs. Ce critère, de même que celui touchant l'engagement dans le travail, renvoie à la notion de construction d'une identité professionnelle et à une conception de l'insertion comme processus de socialisation professionnelle ${ }^{11}$.

En somme, les réponses données par les diplômés sur chacun des thèmes confirment que l'insertion est un processus qui doit être défini en tenant compte de facteurs extérieurs tels que la transformation du marché du travail et l'évolution des types d'emploi. Pour certains diplômés, la stabilité d'emploi renvoie à un emploi stable à temps complet, alors que, pour d'autres, la mouvance du marché du travail et la volatilité des emplois offerts ne sont que des variables avec lesquelles ils composent plutôt sereinement. Les données sur le travail autonome démontrent d'ailleurs que plus une personne est scolarisée, plus elle peut évoluer aisément dans un contexte de mouvance. De prime abord, les diplômés de cycles supérieurs semblent donc plus aptes (du moins, en théorie) à faire face à cette nouvelle réalité.

\section{COMPRENDRE LE PROCESSUS D'INSERTION}

Ce qui nous intéresse, ce n'est pas tant de quantifier l'insertion que de déterminer quels sont les paramètres sur lesquels on peut intervenir pour faciliter et optimiser le processus d'insertion. Pour déterminer ces paramètres, il faut bien comprendre comment se déroule le processus d'insertion du point de vue du diplômé.

11. Ibid., p. 72. 


\section{Circonscrire le processus d'insertion}

Il faut se rappeler d'abord que, pour arriver à circonscrire l'insertion et à la mesurer, on a toujours cherché à en situer le début et la fin. Le processus se définit alors par le passage d'un état initial (p. ex. les études à temps plein) à un autre (p. ex. un emploi permanent à temps plein). Les enquêtes auprès des diplômés cherchent notamment à caractériser la relation entre la formation et l'emploi, en mettant en relation les caractéristiques de la formation et les conditions d'emploi des diplômés quelques années après l'obtention du diplôme. Ces enquêtes permettent d'apporter certaines informations intéressantes sur la situation d'insertion des diplômés. Toutefois, leur objectif n'est pas d'étudier le processus d'insertion. Audelà des informations quantitatives disponibles, on cherche donc à mieux comprendre la finalité de l'insertion. Comment commence-t-elle et quand se termine-t-elle? Quand peut-on dire qu'elle est réussie?

Pour répondre à ces questions, nous avons basé notre analyse du processus d'insertion sur les travaux de Trottier. Celui-ci considère que le début de l'insertion correspond au moment de l'entrée dans la vie active. À ce sujet, Trottier cite les travaux de Vincens, selon lequel «l'insertion commence quand l'individu cesse de partager son temps entre le loisir, les études et le travail non rémunéré pour consacrer du temps à un emploi rémunéré ou à la recherche d'emploi ${ }^{12}{ }$.

Or, le CNCS-FEUQ est d'avis que le début du processus d'insertion ne peut être associé simplement à l'entrée dans la vie active. De fait, l'insertion est un processus non linéaire et difficile à circonscrire: il n'est pas constitué d'étapes bien définies. Comme l'explique Trottier, «[...] l'insertion professionnelle est définie comme un processus qui se déroule sur une période où s'enchevêtrent des situations de recherche d'emploi, de chômage, de formation et d'inactivité (Vincens, 1981, Paul, 1984, Dupaquier et al., 1986). Cette perspective tient compte à la fois des trajectoires professionnelles des individus et de leurs cheminements scolaires $^{13}$.»

La période à laquelle fait référence Trottier doit être perçue comme étant à géométrie variable. Pour le CNCS-FEUQ, l'idée d'insertion intègre l'ensemble de la trajectoire professionnelle, ce qui comprend à la fois

\footnotetext{
12. C. Trottien, R. Cloutier et L. Laforce, «Typologie de l'insertion professionnelle des personnes diplômées de l'université», dans Revue canadienne d'enseignement supérieur, vol. XXIV, n 2, 1994, p. 74.

13. Ibid., p. 73.
} 
l'orientation et la transition formation-emploi. Cette période d'insertion n'est pas reliée à des étapes ou aspects précis de la vie. Elle n'est définie ni par la formation, ni par la recherche d'emploi, ni par les caractéristiques d'emploi, ni par l'avènement d'une vie familiale. Elle est davantage liée à la période durant laquelle est déterminé et accompli le projet professionnel. Comme l'exprime Trottier, «il importe, au plan de l'analyse, de prendre en considération simultanément plutôt que successivement les projets et les cheminements de formation des diplômés de même que leurs projets et leurs trajectoires professionnels ${ }^{14}$ ». Cette façon de voir l'insertion implique notamment que l'orientation des diplômés ne doit pas seulement se faire à la fin du secondaire mais bien tout au long du processus. De même que l'on parle de formation tout au long de la vie, on pourrait dans cette optique parler d'insertion et de réinsertion tout au long de la vie.

\section{Considérer les attentes des diplômés}

Il est relativement simple de comprendre comment s'entame l'insertion telle que nous venons de la décrire. En effet, lorsque l'étudiant commence à envisager son orientation professionnelle, le processus d'insertion débute. Il n'est cependant pas si facile de caractériser où se termine ce processus. Dans l'optique où les diplômés ont aujourd'hui des représentations et des attentes très diverses au regard de l'insertion, la finalité même du processus ne peut plus être simplement identifiée à l'obtention d'un emploi permanent et à temps plein, même si ce dernier est lié aux études. Les représentations des diplômés ainsi que le caractère instable du marché du travail nous poussent fortement à revoir la façon dont on conçoit la fin de l'insertion. L'approche proposée par Vincens il y a plus de 20 ans permet de circonscrire une finalité de l'insertion qui intègre l'aspect subjectif des choix de l'individu. Selon cette approche, l'insertion se termine lorsque l'individu arrête de consacrer du temps à la recherche d'emploi, lorsqu'il obtient un emploi durable (rien ne lui laissant croire qu'il devra changer dans un avenir plus ou moins proche) et qu'il considère vouloir garder cet emploi compte tenu de ce qu'il veut faire et de ce

14. C. Trottier, L. Laforce, R. Cloutier, M. Perron et M. Diambomba, «Planifier ou explorer? Les projets de formation et les stratégies d'insertion professionnelle de diplômés universitaires québécois », dans A. Degenne, M. Lecoutre, P. Lièvre et P. Werquin (dir.), Insertion, transition professionnelle et identification de processus, Centre d'études et de recherche sur les qualifications (Céreq), n 142, mai 1999, p. 361. 
qu'il perçoit des contraintes du marché du travail. On comprend alors que l'insertion n'est réussie que si le diplômé occupe un emploi qui répond à ses attentes.

Par son approche, Vincens a contribué à une redéfinition de l'idée de l'insertion. Il a d'ailleurs inspiré les travaux de Trottier, qui soutient que la finalité de l'insertion consiste en l'atteinte d'une stabilité sur le marché de l'emploi. Pour le CNCS-FEUQ, cette approche rend bien compte de la représentation et des attentes diverses qu'ont les diplômés d'aujourd'hui. Elle permet de tenir compte autant des idées de ceux qui recherchent des emplois stables que de ceux qui arrivent à se stabiliser à travers des emplois instables.

Pour le CNCS-FEUQ, le développement d'une employabilité qui permet de se maintenir stable sur le marché du travail est important pour l'ensemble des diplômés, peu importe leurs attentes. En effet, les emplois à durée indéterminée deviennent parfois instables, étant donné les fermetures d'entreprises et les restructurations constantes de l'économie. En ce sens, la formation continue devient cruciale pour l'employabilité des diplômés, qui peuvent ressentir à tout moment les effets des transformations du marché de l'emploi. En somme, l'obtention d'une situation d'emploi à durée indéterminée nous semble moins indicative d'une insertion réussie que la possibilité de demeurer stable sur le marché de l'emploi.

\section{Le rôle du diplômé, le contexte et les obstacles}

Le fait de mieux circonscrire le processus d'insertion ne permet pas de décrire les obstacles qui empêchent les diplômés de s'insérer conformément à leurs attentes. Pour le faire, il convient de remettre en contexte le cheminement du diplômé au cours du processus d'insertion. Pour Trottier, le diplômé n'est pas seulement le sujet de son insertion, il en est également l'acteur essentiel. Comme il l'explique, le diplômé «a un comportement rationnel dans sa recherche d'emploi et essaie de trouver un emploi en relation avec son projet de vie. [...] Ce dernier n'est cependant pas arrêté définitivement au début de la recherche d'emploi. Il peut se préciser ou être modifié à partir des informations que les personnes diplômées acquièrent en occupant un emploi ou des contraintes qu'elles découvrent dans la concurrence qu'elles affrontent pour y accéder ${ }^{15}$.»

15. C. Trottier, L. Laforce et R. Cloutier, «Les représentations de l'insertion professionnelle chez les diplômés de l’université», op. cit. note 7, p. 62. 
L'analyse de Trottier sous-tend que le diplômé peut agir sur plusieurs aspects de son insertion. À ce sujet, l'auteur va plus loin: il avance que les difficultés auxquelles sont confrontés les diplômés actuels au regard de l'emploi ne sont pas de nature à réduire la possibilité qu'ils ont d'agir sur leur insertion. Selon lui, «il est paradoxal de constater que, malgré les difficultés auxquelles ils sont confrontés sur le marché du travail, ils disposent d'un espace de décisions beaucoup plus large et différencié qu'auparavant. Cet espace est propice à l'élaboration de stratégies variées de formation initiale et d'insertion compte tenu des multiples trajectoires possibles d'entrée dans la vie adulte ${ }^{16} »$.

L'analyse de Trottier se tourne donc vers le diplômé en tant qu'acteur stratégique de son insertion. Dans la section suivante, nous développerons davantage cet aspect fort intéressant. De prime abord, il devient plus clair que les obstacles à l'insertion seront difficiles à généraliser, mais il est aussi possible de constater qu'ils seront liés à la définition et à l'exploration du projet professionnel, puis à l'élaboration d'une stratégie pour y cheminer.

\section{LES STRATÉGIES D'INSERTION}

Cette section reprend l'analyse et les résultats présentés récemment par Trottier ${ }^{17}$. Lapproche trouve notamment sa base théorique dans les concepts présentés par Crozier et Friedberg ${ }^{18}$, qui ont traité de la capacité de l'acteur à faire preuve de rationalité à l'intérieur des systèmes où il évolue. "Mais ils ont aussi mis en relief les limites de la rationalité de l'acteur et le caractère contingent de sa conduite: si celle-ci n'est pas déterminée, ni jamais entièrement prévisible, elle dépend d'un contexte, des opportunités et des contraintes qu'il fournit. L'acteur ne jouit que d'une liberté restreinte et n'est capable que d'une rationalité limitée ${ }^{19}$.» En termes concrets, on peut alors dire que les diplômés peuvent adopter certaines stratégies, bien qu'ils soient limités par leur connaissance restreinte du marché du travail et de la concurrence.

16. C. Trottier, L. Laforce, R. Cloutier, M. Perron et M. Diambomba, «Planifier ou explorer? Les projets de formation et les stratégies d'insertion professionnelle de diplômés universitaires québécois », op. cit. note 51, p. 361.

17. Ibid., p. 359-374.

18. M. Crozier et E. Friedberg, L'acteur et le système, Paris, Éditions du Seuil, 1977, 436 p.

19. C. Trottier, L. Laforce, R. Cloutier, M. Perron et M. Diambomba, «Planifier ou explorer? Les projets de formation et les stratégies d'insertion professionnelle de diplômés universitaires québécois », op. cit. note 51, p. 363. 
Trottier explique très bien que, dans ce contexte, le concept de stratégie ne doit pas être pris au sens traditionnel d'une action planifiée à l'avance. En fait, «le concept de stratégie se réfère à un ensemble de comportements qui témoignent d'une régularité en vue d'atteindre des objectifs qui ne sont pas toujours définis clairement au départ. Ces objectifs peuvent se préciser, changer chemin faisant parce que l'acteur est à l'affût d'opportunités lui permettant d'améliorer sa situation et est préoccupé d'élargir sa marge de liberté par rapport aux contraintes auxquelles il est confronté ou aux comportements des autres acteurs ${ }^{20} »$.

Les différentes approches qu'adoptent les diplômés donnent naissance à des stratégies de natures diverses.

Il est possible de situer les stratégies sur un continuum allant de la stratégie délibérée à la stratégie émergente. La première renvoie a) à un "pattern» de décision caractérisé par un plan explicite pour le futur, élaboré consciemment et intentionnellement avant la prise de décision, et b) à une action méthodique axée sur des objectifs à long terme et l'allocation de ressources pour les réaliser. Dans la stratégie émergente, ce «pattern» se forme graduellement, peut-être même de façon non délibérée, à mesure que les décisions sont prises une à une et que s'effectue une évaluation continuelle des contraintes. Ce type de stratégie est centré davantage sur les moyens que sur les objectifs à long terme, et implique un ajustement graduel des décisions en fonction des contraintes, des opportunités qui se présentent et des ressources disponibles ${ }^{21}$.

Il est intéressant de prendre note que les comportements des diplômés à l'égard de leur insertion, même s'ils ne sont pas toujours planifiés, peuvent suivre un «pattern» stratégique. Dans un processus comme celui de l'insertion, où les étapes s'enchevêtrent et où le cheminement ne peut être que partiellement structuré, la stratégie n'est pas un exercice facile. Selon Trottier,

[...] dans les situations faiblement structurées, le meilleur stratège est celui qui fait preuve d'une réflexivité diffuse, c'est-à-dire d'une capacité de prendre distance par rapport aux situations et au monde qui l'entoure et aux idées reçues, de repérer les diverses possibilités d'action, de les évaluer, d'en anticiper les conséquences explicites et implicites, de mesurer sa propre marge d'autonomie, de changer de registre, d'inventer, de gérer des informations plus ou moins complètes $^{22}$.

\footnotetext{
20. Idem.

21. Ibid., p. 366.

22. Ibid., p. 365 .
} 
Il faut alors retenir qu'il y a un intérêt à planifier davantage les stratégies relatives à l'insertion, mais qu'il faut éviter de se cantonner dans la rigidité au regard de son projet. De fait, le projet professionnel est susceptible d'évoluer, entre autres en fonction des informations obtenues sur le marché du travail. La notion de stratégie s'inscrit bien dans l'optique de la poursuite d'un projet. «Par définition, un projet renvoie à une situation ou à un état que l'on pense atteindre, à une définition d'intention, une projection provisoire de l'avenir. Il ne consiste pas à définir un objectif et à construire un plan précis pour l'atteindre, mais en "une action continue de projection de soi"23.»

Les types de stratèges définis par Trottier permettent de couvrir les différents comportements stratégiques que peuvent adopter les diplômés en ce qui a trait à leur insertion. Ils sont au nombre de neuf:

- Le planificateur résolu: projet précis au départ, non modifié, atteinte des objectifs;

- Le planificateur encore aux études: projet précis au départ, non modifié, en voie de réalisation (le diplômé a poursuivi ses études et n'était pas encore sur le marché du travail au moment de la dernière collecte des données);

- Le planificateur déconcerté: projet précis au départ, non modifié, non-atteinte des objectifs;

- Le planificateur flexible: projet précis au départ, modifié, atteinte des objectifs;

- Le planificateur contrarié: projet précis au départ, modifié, nonatteinte des objectifs;

- L'explorateur contenté: projet flou au départ, explicité par la suite, atteinte des objectifs ;

- L'explorateur encore aux études : projet flou au départ, explicité par la suite, en voie de réalisation (le diplômé a poursuivi ses études et n'était pas encore sur le marché du travail au moment de la dernière collecte de données);

23. Ibid., p. 366. 
- L'explorateur contrarié: projet flou au départ, explicité par la suite, non-atteinte des objectifs ;

- L'explorateur désemparé: projet flou au départ, non explicité par la suite, non-atteinte des objectifs ${ }^{24}{ }$.

Les diplômés de cycles supérieurs n'ont donc pas seulement des attentes et des représentations variées quant à leur avenir professionnel, ils ont aussi des projets bien différents et ils adoptent des stratégies diverses. Afin de mieux situer comment se comportent réellement les diplômés universitaires, Trottier a également procédé à une enquête par entrevues auprès de 61 diplômés de baccalauréat, de 30 diplômés de maîtrise et de 10 diplômés de doctorat. Cette étude montre bien que les diplômés universitaires sont plus planificateurs (70\%).

Il importe de souligner que, parmi les 73 planificateurs, 32 ont modifié leur projet. La plupart l'ont fait après leurs études soit parce qu'ils ne trouvaient pas d'emploi dans un domaine lié à leur formation, soit parce qu'ils n'aimaient pas le type d'emploi auquel les avait conduits leur formation et qu'ils avaient développé de nouveaux intérêts, soit parce qu'ils ont découvert sur le marché du travail des opportunités qu'ils n'avaient pas anticipées, soit parce qu'ils ont dû se retirer du marché du travail pour des raisons de santé. [...] De ce point de vue, il y a plus de planificateurs qui ont modifié leur projet (32, en majorité suite à leur entrée sur le marché du travail) que d'explorateurs (28) qui sont parvenus à définir ou à expliciter un projet (25). Ces résultats témoignent du caractère émergent des stratégies d'insertion, et illustrent que celles-ci peuvent être définies de façon graduelle même dans le cas de diplômés qui avaient un projet précis au départ ${ }^{25}$.

Une des raisons qui expliquent les stratégies émergentes et les modifications de projet une fois sur le marché du travail est la mauvaise connaissance qu'ont les diplômés du marché du travail. Les observations faites par Trottier tendent à confirmer cette analyse: en effet, les diplômés en cheminement continu ${ }^{26}$, qui ont une bien meilleure connaissance du marché du travail où ils s'orientent, planifient davantage que les autres.

24. Ibid., p. 368.

25. Ibid., p. 370.

26. Les diplômés en cheminement continu sont ceux qui poursuivent des études en lien avec le travail qu'ils occupent ou qu'ils occupaient, notamment pour se perfectionner. Par ailleurs, les diplômés en réinsertion sont ceux qui reviennent aux études pour acquérir une formation différente, avec l'intention d'effectuer un changement de carrière. 
Si on compare les diplômés qui en étaient à leur première insertion avec ceux qui étaient en réinsertion ou en cheminement continu, les diplômés qui ont eu le plus tendance à planifier leur stratégie sont ceux qui étaient en cheminement continu (9/10) et qui s'engageaient dans des études tout en étant déjà insérés sur le marché du travail. Tout se passe comme si «l'investissement» qu'ils avaient décidé de faire dans leur formation tout en continuant à travailler les avait incités à préciser leur projet au départ pour en maximiser le rendement. Par contre, seulement la moitié des diplômés en réinsertion ont fait appel à une stratégie planifiée ${ }^{27}$.

Cette dernière observation démontre bien le caractère souvent émergent des stratégies de réinsertion.

D'après le CNCS-FEUQ, on peut certainement se questionner sur le manque d'orientation relativement au projet professionnel. Une construction peu consciente du projet pose notamment un problème pour le développement de compétences visant à favoriser une stabilité accrue sur le marché de l'emploi. La question sera plus explorée dans le chapitre suivant.

27. C. Trottier, L. Laforce, R. Cloutier, M. Perron et M. Diambomba, «Planifier ou explorer? Les projets de formation et les stratégies d'insertion professionnelle de diplômés universitaires québécois », op. cit. note 51, p. 370. 


\section{Chapitre}

\section{FAVORISER L'INSERTION OPTIMALE DES DIPLÔMÉS DES CYCLES SUPÉRIEURS}

\section{COMMENT INTERVENIR SUR L'INSERTION ?}

Dès le départ, nous nous étions proposés de définir comment on pouvait intervenir pour favoriser une insertion optimale des diplômés. Nous cherchions à comprendre le processus qui leur permet de réussir leur insertion. L'analyse présentée au chapitre 3 nous a permis de faire quelques constats essentiels. D'une part, il a été démontré que la réussite de l'insertion, du point de vue des diplômés, n'était pas un concept unique. Les diplômés ont parfois des représentations fort différentes de ce qu'est l'insertion. Pour certains, le concept est lié à la stabilité de l'emploi, alors que pour d'autres il dépend de l'employabilité du diplômé. Certains se satisfont simplement d'un emploi qui assure un revenu, alors que d'autres perçoivent leur travail comme un moyen d'expression, une occasion de se réaliser. Bref, la pluralité des représentations signifie que la réussite de l'insertion est un concept à géométrie variable.

Dans cette optique, il est complexe pour qui s'intéresse à faciliter l'insertion de fixer quelque objectif que ce soit en ce qui concerne la finalité de l'insertion. On peut difficilement proposer des mesures visant à 
amener l'individu vers un état d'insertion optimal, puisque cet état varie selon les projets qu'élaborent les diplômés. En fait, au cours du processus d'insertion, nous avons vu que l'individu agit, de manière planifiée ou non, de telle sorte qu'il progresse plus ou moins efficacement vers la réalisation d'un projet professionnel. Ce projet professionnel est d'autant plus unique qu'il s'intègre au projet de vie des diplômés, lequel comprend d'autres facettes (famille, loisir, etc.) dont l'importance peut évoluer dans le temps.

Étant donné la difficulté de circonscrire le processus d'insertion et d'en dégager un modèle optimal commun, il convient de redéfinir les éléments fondamentaux qui composent tout projet d'insertion. En nous basant sur les études existantes, nous dégageons trois éléments qui ne s'avèrent pas indépendants, mais qui sont plutôt en synergie durant tout le continuum du projet professionnel:

- La construction d'un projet professionnel, par l'orientation et l'exploration professionnelles, qui commence dès le secondaire et se poursuit, de manière latente ou active, tout au long des processus de formation et d'insertion;

- Lélaboration et la mise en œuvre d'une stratégie planifiée ou émergente, qui renvoie aux actions que prend le diplômé pour répondre aux exigences de son projet, comme le développement de compétences, l'acquisition d'expérience, etc.;

- L'expérimentation d'une stabilisation sur le marché du travail, qui permet de valider le projet et la stratégie, en les réévaluant de manière dynamique selon l'évolution de son projet de vie et en fonction des contraintes propres au marché du travail.

Ces éléments fondamentaux constituent les aspects sur lesquels on peut agir pour aider le diplômé dans son processus d'insertion. Une action plus efficace sur chacun de ces éléments permet de favoriser l'insertion peu importe les représentations qu'en ont les diplômés. Par ailleurs, l'importance des éléments varie selon les représentations. Par exemple, les diplômés qui considèrent le travail comme un moyen de réalisation de soi accorderont plus d'importance au troisième élément (l'expérimentation) que les diplômés qui ne souhaitent qu'une participation au marché du travail. 


\section{LE CNCS-FEUQ RECOMMANDE DONC:}

Que les interventions visant à améliorer l'insertion se basent sur une conception de la transition vers l'emploi qui comprend les trois éléments fondamentaux suivants:

- La construction d'un projet professionnel, par l'orientation et l'exploration professionnelles, qui commence dès le secondaire et se poursuit, de manière latente ou active, tout au long des processus de formation et d'insertion;

- L'élaboration et la mise en œuvre d'une stratégie planifiée ou émergente, qui renvoie aux actions que prend le diplômé pour répondre aux exigences de son projet, comme le développement de compétences, l'acquisition d'expérience, etc.;

- L'expérimentation d'une stabilisation sur le marché du travail, qui permet de valider le projet et la stratégie, en les réévaluant de manière dynamique selon l'évolution de son projet de vie et en fonction des contraintes propres au marché du travail.

\section{LES FACTEURS STRUCTURAUX INFLUENÇANT L'INSERTION}

Nous avons vu au chapitre 9 que les jeunes diplômés universitaires ne se sentent pas toujours désemparés devant l'instabilité des situations d'emploi d'aujourd'hui. Une majorité d'entre eux ont appris à vivre avec ce spectre. Plusieurs abordent même cette réalité avec optimisme, car ils considèrent que leur préparation leur permettra de se stabiliser sur le marché du travail. Le CNCS-FEUQ croit qu'il serait judicieux, dans un contexte d'instabilité qui ne semble pas vouloir se résorber, d'outiller encore mieux les jeunes diplômés universitaires pour y faire face le plus sereinement possible.

Par ailleurs, le CNCS-FEUQ juge qu'il faut toujours consacrer de grands efforts à stabiliser les emplois. Plusieurs jeunes recherchent des emplois stables, parce qu'ils sont incapables de vivre dans un contexte de mouvance constant ou parce qu'ils arrivent à une période de leur vie où ils désirent établir des bases solides, par exemple fonder une famille. De plus, plusieurs études ${ }^{1}$ font état du contexte parfois difficile dans lequel

1. Pour plus de détails, se référer à: Conseil permanent de la jeunesse, Emploi atypique et précarité chez les jeunes: une main-d'œuvre à bas prix, compétente et jetable, avis, Québec, 2001, $114 \mathrm{p}$. 
se trouvent les jeunes diplômés sur le marché du travail. Les jeunes les moins qualifiés sont en fait les plus touchés par cette précarité. Devant l'instabilité des emplois, plusieurs jeunes choisissent l'entrepreneuriat ou le travail autonome, mais la formule ne réussit pas à tous. Qu'on parle de diplômés de cycles supérieurs ou de toute autre catégorie de jeunes travailleurs, le maintien de la stabilité d'emploi est important. Le CNCSFEUQ juge donc que différentes mesures à caractère structurel doivent être adoptées en priorité. Parmi celles-ci, notons celles du Conseil permanent de la jeunesse qui a déjà fait état de plusieurs sources de précarité et d'instabilité d'emploi chez les jeunes, soit le très faible renouvellement de la fonction publique québécoise ${ }^{2}$, l'imposition de clauses orphelins ${ }^{3}$ et le champ restreint d'application de la Loi sur les normes du travail ${ }^{4}$. Jusqu'à maintenant, les efforts consentis par le gouvernement au sujet de ces problématiques n'ont pas permis de modifier la donne.

Dans l'intérêt de l'ensemble des jeunes, le gouvernement doit favoriser la stabilité d'emploi et, de surcroît, trouver de nouveaux moyens d'assurer une certaine stabilité aux jeunes dans un marché du travail instable.

La particularité des diplômés de cycles supérieurs est probablement d'être les moins touchés par l'instabilité d'emploi. Les études démontrent qu'ils peuvent se stabiliser plus facilement sur le marché du travail. Cependant, rien ne garantit que les emplois qu'ils comblent mettent à profit leurs aptitudes et compétences au regard de la recherche, du développement et de l'innovation. Voilà les raisons qui ont motivé le CNCSFEUQ à introduire le concept d'insertion optimale. Celui-ci permet, comme nous le verrons ci-après, de considérer des interventions qui visent à améliorer les retombées du processus d'insertion.

2. Conseil permanent de la jeunesse, L’accès des jeunes à la fonction publique québécoise: Place à la relève!, avis, Québec, Le Conseil, 1999, 96 p.

3. Conseil permanent de la jeunesse, Interdire une fois pour toutes les clauses "orphelin", présentation à la Commission parlementaire de l'économie et du travail sur le projet de loi portant sur les disparités de traitement, Québec, Le Conseil, 1999, 10 p.

4. Conseil permanent de la jeunesse, Un vrai défi collectif: de nouvelles normes pour un nouveau travail!, mémoire présenté au ministre du Travail en réaction au document de consultation: «Revoir les normes du travail - Un défi collectif!», Québec, Le Conseil, 2002, 19 p. 


\section{COMMENT CONCEVOIR L'INSERTION OPTIMALE ?}

Parler d'insertion optimale, c'est évoquer une hausse des exigences par rapport à l'insertion. On ne désire plus seulement que les diplômés aient un type d'emploi donné ou que leur situation réponde à un standard. Il faut que l'insertion engendre des retombées positives optimisées pour l'individu et pour la société. Ainsi, nous avons défini deux objectifs au regard de l'insertion optimale.

Du point de vue de l'individu, l'insertion est optimale lorsque:

- le diplômé de cycles supérieurs a développé les compétences et acquis le bagage nécessaire pour s'assurer une stabilité sur le marché du travail dans un type d'emploi relié à son projet et qui lui permet de s'épanouir.

Du point de vue de l'apport social, culturel et économique, l'insertion est optimale lorsque:

- le diplômé de cycles supérieurs peut contribuer à l'innovation et au développement par la réalisation de son plein potentiel.

Le premier objectif découle clairement des analyses du chapitre 9 et se base sur la vision stratégique qui place le diplômé au centre de son processus d'insertion. Il rejoint les trois aspects des représentations de l'insertion définis au chapitre 9: la stabilité sur le marché du travail, la relation formation-emploi et le développement d'une identité professionnelle. Par ailleurs, le second objectif est un élément extérieur au processus d'insertion qui rejoint l'enjeu de l'innovation et du développement. Il repose sur la mise en valeur de compétences propres aux diplômés de cycles supérieurs, notamment les aptitudes acquises en recherche. Il peut aussi s'agir de connaissances et de compétences qui ne relèvent pas directement de la formation aux cycles supérieurs, mais qui sont acquises par l'expérience ou la formation continue.

Les chapitres 10, 11 et 12 reprennent les deux objectifs que nous venons d'énoncer et proposent des axes d'intervention qu'il faut considérer pour favoriser une insertion optimale, tant du point de vue du diplômé que des retombées pour la société. 


\section{RECOMMANDATIONS}

Que le CNCS-FEUQ fasse la promotion de l'importance de l'insertion optimale des diplômés de cycles supérieurs comme moyen de favoriser leur stabilisation sur le marché de l'emploi, de même que le développement et l'innovation dans les différents milieux de travail.

Que le CNCS-FEUQ défende que l'insertion est optimale lorsque:

Sur le plan de l'individu,

1. Le diplômé de cycles supérieurs a développé les compétences et acquis le bagage nécessaire pour s'assurer une stabilité sur le marché du travail dans un type d'emploi en relation avec son projet.

Sur le plan de l'apport social, culturel et économique,

2. Le diplômé de cycles supérieurs peut contribuer au développement et à l'innovation par la mise en valeur de son plein potentiel. 


\section{Chapitre}

\section{L'ÉLABORATION D'UN PROJET PROFESSIONNEL ET L'ORIENTATION}

La poursuite d'un projet professionnel et l'établissement d'une stratégie pour acquérir le bagage nécessaire à l'emploi souhaité sont des conditions permettant de faciliter l'insertion optimale. Nous avons conclu au chapitre 8 que le meilleur stratège était celui qui pouvait prendre du recul à différents moments par rapport à son projet d'insertion pour évaluer les possibilités qui s'offrent à lui. Cela implique toutefois la construction d'un projet professionnel. Ainsi, dans un premier temps, il importe de centrer l'action sur l'orientation et l'exploration professionnelles.

\section{POUR:}

Que le diplômé de cycles supérieurs développe les compétences et acquière le bagage nécessaire pour s'assurer une stabilité sur le marché du travail dans un type d'emploi relié à son projet et qui lui permet de s'épanouir.

\section{ON DEVRAIT INTERVENIR SUR:}

La construction d'un projet professionnel, par l'orientation et l'exploration professionnelles, qui commence dès le secondaire et se poursuit, de manière latente ou active, tout au long des processus de formation et d'insertion. 


\section{LE MANQUE CHRONIQUE DE RESSOURCES CONSACRÉES À L'ORIENTATION}

Le processus d'orientation est peu développé au Québec, ce qui laisse à la discrétion de chacun le soin de situer son projet professionnel. En 1995, l'Ordre professionnel des conseillers et conseillères d'orientation du Québec estimait que le ratio orienteur-élèves au secondaire variait de 1 pour 1200 à 1 pour 1700: il était de 1 pour 875 en 1989¹. Depuis, la situation ne semble pas s'être améliorée en ce qui concerne la disponibilité des ressources professionnelles. Récemment, le ministère de l'Éducation du Québec implantait une nouvelle approche d'intervention, l'école orientante, pour intégrer la dimension de l'exploration et de l'orientation professionnelle tout au long du parcours secondaire. L'approche est générale: elle favorise notamment la réalisation d'activités diverses permettant de construire une identité professionnelle et vise à démontrer aux jeunes la pertinence des contenus de cours en lien avec leur utilité pratique.

Bien qu'elle se fonde sur l'importance de l'orientation, l'approche de l'école orientante est très critiquée. Les spécialistes en orientation jugent qu'elle est inadéquate, car elle dilue encore le rôle des professionnels en orientation alors qu'il devrait être renforcé. L'approche repose grandement sur l'intervention d'enseignants qui n'ont pas de formation dans le domaine de l'orientation. Aussi, on mise de plus en plus sur le recours à l'information, sur les banques de données sur les carrières. Comme le mentionnent plusieurs intervenants en orientation, il ne suffit certainement pas de mettre les étudiants indécis (et ils le sont presque tous) devant une banque de données pour qu'ils réussissent à s'orienter ${ }^{2}$. Les adolescents sont en processus de développement: ils cherchent à se construire une identité personnelle ${ }^{3}$. Il n'est pas aisé de les faire cheminer vers un choix de carrière tout en les accompagnant dans le développement de leur identité.

1. B. Breton, «Les étudiants perdent à LOTO-CO - Jusquà trois mois d'attente pour obtenir un rendez-vous avec un conseiller d'orientation", dans L'orientation, Ordre professionnel des conseillers et conseillères d'orientation du Québec, vol. 11, nº 2, 1998, p. 17-19.

2. Idem.

3. J. Paquin, «L'école orientante: pour une approche de l'orientation adaptée au développement de l'adolescent», dans L'orientation, Ordre professionnel des conseillers et conseillères d'orientation du Québec, vol. 12, n 1, 1999, p. 5-10. 
Pour le CNCS-FEUQ, comme pour la majorité des intervenants, il est clair que les professionnels en orientation doivent être plus nombreux et avoir le temps d'aider vraiment les élèves, ce qui n'est pas le cas actuellement. Comme l'illustre Bleau, on a parfois tendance à s'attaquer aux difficultés d'insertion sans pour autant toucher le problème, c'est-àdire le développement de l'identité. Or, le développement de l'identité est fondamental non seulement pour l'orientation au secondaire, mais aussi pour assurer une base solide par la suite. Bleau illustre ce propos en reprenant une citation de Condamin qui s'inspire du conte des Trois petits cochons: «les maisons de paille sont peut-être plus économiques à construire mais elles n'offrent guère de sécurité... le développement de l'individu se construit comme une seule et même maison, avec ses bases, ses murs et son toit et [...] il est inutile d'essayer de faire un toit lorsqu'il n'y a ni mur solides, ni bases établies ${ }^{4} . . . »$. Il est donc important d'investir pour rendre disponibles les ressources professionnelles.

D'ailleurs, les investissements en orientation sont rapidement récupérables. En effet, on rapporte qu'environ $60 \%$ des diplômés du collégial avaient une idée peu ou pas précise de leur choix de carrière à la fin du secondaire. En conséquence, un étudiant sur trois change de programme au cours de ses études collégiales ${ }^{5}$.

\section{L'ORIENTATION, UN PROCESSUS CONTINU}

Alors qu'au secondaire plusieurs jeunes ne sont pas prêts à élaborer un projet professionnel, on ne semble pas prévoir un processus d'orientation bien soutenu par la suite. Il existe bien des services d'orientation au collégial et à l'université, mais, dans plusieurs établissements, ils sont peu développés ou simplement inexistants. Au collégial, par exemple, le nombre d'étudiants par orienteur est généralement supérieur à ce qu'on retrouve au secondaire. Au début des années 1990, le ratio orienteurétudiants y était de 1 pour $2000^{6}$.

4. M. Bleau, «L'orientation? On peut aller plus loin!», dans L'orientation, Ordre professionnel des conseillers et conseillères d'orientation du Québec, vol. 13, $\mathrm{n}^{\circ} 1,2000$, p. 8-11.

5. Idem.

6. Conseil permanent de la jeunesse, Une «cure de jeunesse» pour l'enseignement collégial, avis, Québec, Le Conseil, 1992, p. 36. 
Dans ce contexte, il n'est pas étonnant de constater que le système de formation universitaire n'encourage pas les étudiants à établir les possibilités professionnelles qui s'offrent à eux et à en explorer les différents aspects. Or, à mesure que les étudiants avancent dans leur formation, ils modifient et précisent les possibilités qui s'offrent à eux. Si, dans certaines disciplines professionnelles, le parallèle entre la formation et l'emploi est clair, il demeure que les étudiants connaissent peu les réalités et l'étendue des possibilités qu'ils ont. Cela est d'autant plus vrai dans les programmes qui ne mènent pas à une profession ou à un emploi déterminé.

Comme les données du chapitre 8 l'indiquent, une proportion non négligeable d'universitaires et d'étudiants aux cycles supérieurs poursuivent leurs études en repoussant toujours à plus tard les réflexions professionnelles. Comme le souligne Trottier après avoir mené une enquête par interview sur l'insertion "[...] nous avons constaté que certains diplômés, même au niveau doctoral, ne se sont préoccupés de leur entrée sur le marché du travail qu'à la fin des études qu'ils avaient entreprises. Ils n'étaient pas particulièrement pressés d'arrêter un projet précis se laissant le temps d'explorer ${ }^{7} »$.

Il n'est pas surprenant que les étudiants universitaires prennent plus de temps qu'auparavant à se stabiliser sur un marché du travail, où les emplois sont plus instables que jamais, si on ne les incite pas à mieux se préparer durant leur formation. Les périodes transitoires de précarité qui caractérisent l'insertion des jeunes d'aujourd'hui constituent effectivement une période propice aux questionnements et à l'exploration professionnelle. Cependant, il serait fortement souhaitable que les diplômés puissent réduire cette période d'exploration en se préparant adéquatement.

De plus, il est reconnu que le développement d'objectifs professionnels constitue un important facteur de motivation pour les étudiants. Certains d'entre eux redoutent simplement leur entrée sur le marché du travail. Il s'agit d'une inquiétude souvent due à l'incertitude au regard de l'avenir professionnel, à une perception négative de l'emploi ou à une connaissance restreinte des possibilités professionnelles. Qui plus est,

7. C. Trottier, L. Laforce, R. Cloutier, M. Perron et M. Diambomba, « Planifier ou explorer? Les projets de formation et les stratégies d'insertion professionnelle de diplômés universitaires québécois », dans A. Degenne, M. Leconte, P. Lièvre et P. Werquin (dir.), Insertion, transition professionnelle et identification du processus, Centre d'études et de recherche sur les qualifications (Céreq), n 142, mai 1999, p. 369. 
plusieurs universitaires ont encore beaucoup à faire pour cerner leur identité personnelle et, conséquemment, les perspectives professionnelles qui leur conviennent. Le manque de projet ou de perspectives professionnelles est un facteur évident de prolongement des études. Il est dans l'intérêt du gouvernement, des universités et des étudiants que soit intégrée à la formation universitaire une exploration des possibilités professionnelles qui s'offrent au futur diplômé.

\section{LES MOYENS APPROPRIÉS POUR ASSURER UN PROJET D'INSERTION OPTIMAL}

Avec la mouvance du marché de l'emploi, les services d'information sur les carrières et sur l'emploi ont connu un développement fulgurant. Il existe aujourd'hui de multiples publications qui s'adressent aux étudiants et aux diplômés des différents ordres d'enseignement. Le développement d'Internet a aussi permis la prolifération des services accessibles en ligne. On s'imagine alors qu'il suffit de consulter les différentes banques d'information pour déterminer les perspectives professionnelles les plus adaptées à nos attentes et pour s'assurer une meilleure insertion. Or les études indiquent bien que la construction du projet personnel revêt une certaine complexité.

Il est certain que la connaissance des possibilités professionnelles et du marché de l'emploi s'accroît avec le niveau de formation, mais elle se complexifie également. Les emplois exigeant une formation universitaire impliquent souvent des responsabilités supérieures, elles peuvent être régies par des lois professionnelles et des codes déontologiques. La notion de responsabilité professionnelle est également liée aux considérations éthiques et aux retombées que peut entraîner la pratique professionnelle. En outre, les tâches sont parfois moins bien circonscrites et demandent une plus grande autonomie. Dans ces conditions, les diplômés universitaires et, à plus forte raison, les diplômés de cycles supérieurs ont besoin de se questionner davantage sur leurs intérêts professionnels.

Pour situer les types d'emploi, les secteurs d'activité et les milieux de travail qui conviennent, l'étudiant doit trouver réponse à de nombreuses questions. Quelles compétences a-t-il? Lesquelles lui manque-t-il?Quelles tâches veut-il faire? Quelle contribution à la société, à l'employeur, veut-il apporter? Quels impacts sociaux et éthiques la profession qu'il envisage implique-t-elle? Les milieux de travail qui l'intéressent correspondent-ils à ses valeurs? 
Cet exercice de questionnement est nécessaire afin de conscientiser l'étudiant afin qu'il élabore un projet professionnel réaliste. Celui-ci peut être modifié et adapté en chemin, mais il permet tout de même de se préparer au type d'emploi recherché. Cette préparation s'avère essentielle à l'élaboration d'une stratégie d'insertion, laquelle peut reposer sur l'acquisition de différentes compétences, sur l'implication auprès de différents réseaux ou organismes, ou sur la recherche d'expériences de travail pertinentes.

Or, même s'il existe des services de placement et d'orientation dans la majorité des universités, ceux-ci ne sont pas toujours bien développés. Ceux des grandes universités le sont, mais ils doivent composer avec des budgets restreints qui limitent les possibilités. Par ailleurs, dans les universités en région, les services de placement sont parfois inexistants ou embryonnaires. Les services sont surtout consultés par les services de placement en emploi au moment de l'obtention du diplôme. Conséquemment, une large proportion d'étudiants ne consultent pas et laissent leur projet professionnel indéfini.

Dans un premier temps, le CNCS-FEUQ croit que la démarche d'exploration professionnelle doit être encouragée chez l'ensemble des étudiants. Bien qu'il s'agisse d'un cheminement personnel, il mérite d'être favorisé et soutenu. Son importance dans une optique d'insertion optimale justifie qu'on y consacre un effort systématique. Ainsi, nous croyons qu'il faut favoriser, dans chaque formation universitaire, une démarche personnelle encadrée d'orientations et d'explorations professionnelles de manière à ce que chaque étudiant puisse: réévaluer ses projets professionnels en fonction de ses intérêts, de ses valeurs et de ses autres projets de vie; revoir les aptitudes et les compétences qu'il possède; reconsidérer les possibilités professionnelles qui s'offrent à lui; déterminer les exigences que requièrent ces possibilités; et établir les choix qui semblent les plus appropriés.

Dans les services universitaires, on constate souvent que les étudiants aux cycles supérieurs qui consultent n'ont pas une idée très définie de ce qui les distingue des autres. Ils ne sont pas non plus conscients des compétences requises par les employeurs ou des types d'emploi qu'ils visent. De plus, on remarque, à quelques exceptions près, qu'il n'existe pas de services s'adressant spécialement aux étudiants de cycles supérieurs. Le CNCS-FEUQ croit que les caractéristiques des compétences et de l'emploi aux cycles supérieurs devraient pousser les universités à consacrer 
des efforts particuliers à l'orientation et au placement des étudiants aux cycles supérieurs. Afin de faciliter l'implantation de services adaptés pour les étudiants de cycles supérieurs, le CNCS-FEUQ croit que les universités doivent travailler en concertation pour définir les besoins particuliers et les services nécessaires. Un groupe de travail spécial mandaté par la Conférence des recteurs et principaux des universités du Québec (CRÉPUQ) pourrait par exemple être chargé de cette tâche.

\section{RECOMMANDATIONS}

Que l'on favorise, dans chaque formation universitaire, une démarche personnelle encadrée d'orientations et d'explorations professionnelles et dont les objectifs seraient :

- de réévaluer les intérêts et les valeurs de l'étudiant en considérant ses autres projets de vie;

- de revoir les aptitudes et les compétences qu'il possède;

- de reconsidérer les possibilités professionnelles qui s'offrent à lui;

- de déterminer les exigences que requièrent ces possibilités;

- d'établir les choix qui semblent les plus appropriés selon ses valeurs et ses intérêts.

Que les services de placement et d'orientation professionnelle universitaires accordent une attention particulière aux besoins propres aux étudiants de cycles supérieurs et qu'ils mettent sur pied des services pour y répondre.

Que les universités travaillent en concertation pour définir les besoins particuliers et les services nécessaires aux étudiants de cycles supérieurs.

Que le gouvernement assure une partie du financement des services de placement et d'orientation professionnelle afin qu'ils puissent contribuer plus activement à favoriser l'insertion optimale des diplômés de cycles supérieurs.

Que les universités favorisent une meilleure connaissance des milieux professionnels auxquels s'intéressent les diplômés, par exemple en promouvant des activités de parrainage ou des stages de formation aux cycles supérieurs. 
En conclusion, le CNCS-FEUQ tient à mentionner qu'il est conscient que les propositions faites dans ce texte nécessitent un financement accru des services de placement et d'orientation. À ce titre, les retombées découlant de l'insertion optimale des diplômés universitaires justifient pleinement un accroissement substantiel des ressources allouées. 


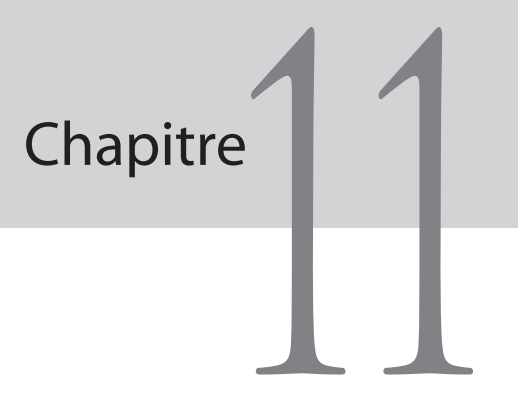

\section{LA FORMATION ET LE DÉVELOPPEMENT DES COMPÉTENCES}

Les étudiants aux cycles supérieurs ont souvent la réputation d'être très spécialisés et de ne pas avoir la polyvalence requise dans les milieux de travail. Cette perception a souvent été évoquée par les employeurs et soulève trois questionnements. D'abord, il est possible de s'interroger sur le bien-fondé de cette perception qu'ont les employeurs, sur leur compréhension des compétences que possèdent les diplômés de cycles supérieurs, de même que sur leur capacité de les mettre en valeur.

Les deux autres questionnements soulevés concernent l'efficacité de la formation universitaire aux cycles supérieurs, ainsi que le développement, en parallèle à celle-ci ou après celle-ci, de compétences particulières et de connaissances pratiques. Ces aspects font l'objet du présent chapitre et concernent l'approche stratégique du processus d'insertion. 
POUR:

Que le diplômé de cycles supérieurs développe les compétences et le bagage nécessaire pour s'assurer une stabilité sur le marché du travail dans un type d'emploi relié à son projet et lui permettant de s'épanouir.

\section{ON DEVRAIT INTERVENIR SUR:}

L'élaboration et la mise en œuvre d'une stratégie planifiée ou émergente, qui renvoie aux moyens que prend le diplômé pour répondre aux exigences de son projet, comme le développement de compétences, l'acquisition d'expérience, etc.;

L'expérimentation d'une stabilisation sur le marché du travail, qui permet de valider le projet et la stratégie, en les réévaluant de manière dynamique selon l'évolution de son projet de vie et en fonction des contraintes propres au marché du travail.

\section{UNE ADAPTATION DE LA FORMATION À LA RECHERCHE DANS LES UNIVERSITÉS}

L'adéquation de la formation au troisième cycle à titre de phase préparatoire à l'entrée sur le marché du travail est sérieusement remise en question. En effet, la formation a peu évolué récemment et les étudiants au doctorat sont toujours formés d'abord pour entreprendre une carrière universitaire $^{1}$. Le Groupe de recherche institutionnel sur les effectifs étudiants soutient qu'il est impératif de répondre à la question suivante: y a-t-il des débouchés pour les titulaires de doctorat en dehors de l'enseignement supérieur? Si la réponse est oui, il importe de s'interroger sur la pertinence de revoir la formation qui est offerte aux étudiants de troisième cycle.

Le CNCS-FEUQ a déjà pris position sur l'adéquation entre la formation universitaire à la recherche et les aptitudes requises pour effectuer de la recherche à l'extérieur du cadre universitaire. Dans un avis déposé lors des audiences publiques sur la politique québécoise de l'innovation, le CNCS-FEUQ s'était prononcé comme suit:

Lépanouissement personnel et l'apport socioprofessionnel des diplômés nécessitent une formation de base qui permet d'en faire des citoyens critiques, mais leur permet également d'acquérir les

1. Groupe de recherche institutionnel sur les effectifs étudiants, L'insertion professionnelle des personnes diplômées de 1993, Relance 1996, document n 4, Université Laval, Québec, avril $1997,188 \mathrm{p}$. 
compétences et qualités requises par les différents milieux où ils évolueront. Actuellement, au Québec, on profite très peu des aptitudes en recherche des étudiants chercheurs diplômés, que ce soit dans les entreprises, ou à plus forte raison dans les coopératives ou les organismes communautaires. De fait, la formation à la recherche prépare à la recherche académique, qui diffère cependant de la recherche appliquée et du développement effectué en entreprise ou de la recherche empirique et expérimentale qui est propre au secteur communautaire. Or, ce sont dans ces milieux, qui ont des besoins en recherche variés, que devront évoluer les étudiants. Les différents besoins en recherche appellent évidemment des méthodes et des habitudes de recherche différentes qu'il faut enseigner. Une culture de développement équilibré au Québec se base donc sur l'apport de jeunes chercheurs capables de s'adapter aux types de travail exigés par les différents milieux, et ayant une conscience sociale et éthique ${ }^{2}$.

Pour le CNCS-FEUQ, l'adaptation de la formation à la recherche aux nouvelles réalités qui attendent les diplômés de cycles supérieurs est un impératif. Les données démontrent que la carrière de professeur constitue la destination d'une minorité de diplômés de doctorat. En fait, si l'on considère également les finissants de maîtrise, une faible minorité des diplômés de cycles supérieurs se consacre à la recherche universitaire. Plusieurs évoluent dans d'autres milieux, où ils effectuent des activités de recherche et de développement de nature variée. Sans diluer l'apprentissage relatif aux carrières universitaire, il faut indéniablement considérer qu'il existe différents profils de carrière en recherche, lesquel nécessitent des compétences générales diverses. Qu'on fasse des études ou des recherches dans un ministère, dans les milieux communautaires, dans une entreprise de haute technologie ou à l'université, certaines compétences de base sont essentielles. Toutefois, une part des compétences requises diffère sensiblement d'un milieu à l'autre, tout comme les objectifs qui sont poursuivis. Il est du ressort de l'université de donner une formation de base différenciée. Une maîtrise de type recherche, mais à vocation plus professionnelle, apparaît tout indiquée à cet effet, alors que la formation doctorale peut demeurer axée sur la recherche scientifique ou universitaire tout en élargissant les compétences générales acquises. Par la suite ou parallèlement, un apprentissage pratique et l'acquisition de compétences propres aux différents milieux de travail peuvent se poursuivre en dehors de la formation universitaire.

2. CNCS-FEUQ, Politique scientifique: pour une vue d'ensemble, avis, Fédération étudiante universitaire du Québec, 2000, p. 24-25. 
Pour le CNCS-FEUQ, il importe dans ce nouveau contexte de responsabiliser les étudiants chercheurs sur les considérations éthiques et la conscience sociale où l'application de la recherche devient de plus en plus importante. La recherche, qu'on caractérise comme étant de plus en plus socialement distribuée, a de fait un impact important sur le développement d'une multitude de secteurs de la société. Dans ces divers secteurs, il faut souvent faire de la recherche multidisciplinaire en visant certains objectifs pratiques. Pour les étudiants de cycles supérieurs, une initiation à la recherche en milieu de travail semble d'une grande pertinence d'un point de vue formatif.

Si certains aspects de la formation peuvent être réalisés par l'intermédiaire de partenariats de recherche, le CNCS-FEUQ tient à préciser que des mécanismes de contrôle doivent être envisagés pour éviter les situations potentielles de conflits. Les étudiants aux cycles supérieurs peuvent se retrouver en fâcheuse position dans de tels cas, ce qui a poussé le CNCS-FEUQ à étudier de plus près ces situations.

En somme, il est temps de reconsidérer officiellement la formation à la recherche aux cycles supérieurs. Déjà, cette question a été mise à l'ordre du jour par l'Association canadienne des études supérieures (ACÈS), qui regroupe l'ensemble des doyens d'études supérieures, les étudiants de cycles supérieurs et plusieurs autres partenaires pertinents à la grandeur du Canada. Le CNCS-FEUQ prévoit participer à ces réflexions. Au Québec, il importe toutefois d'entamer des démarches concrètes visant à établir les objectifs que doit poursuivre la formation à la recherche. Les étudiants de cycles supérieurs invitent donc la Conférence des recteurs et principaux des universités du Québec (CRÉPUQ), l'Association des doyens d'études supérieures au Québec (ADÉSAQ) et, ultimement, le ministère de l'Éducation à repenser la formation à la recherche et à suggérer des modifications applicables à court et à moyen terme.

\section{RECOMMANDATIONS}

Que soit revue la formation à la recherche afin d'intégrer l'acquisition d'un esprit critique et d'une conscience civique et sociale, de même qu'un apprentissage des méthodes de recherche propres aux besoins des milieux pratiques.

Que l'apprentissage d'une méthodologie de recherche axée sur l'atteinte d'objectifs fasse aussi partie de la formation et que les expériences pratiques permettent de l'appliquer. 


\section{LE DÉVELOPPEMENT DES COMPÉTENCES DANS UN OBJECTIF STRATÉGIQUE DE PLANIFICATION PROFESSIONNELLE}

Certaines compétences et aptitudes complémentaires, qui varient selon les milieux de travail, sont recherchées, voire nécessaires pour permettre aux diplômés de cycles supérieurs d'être efficaces en emploi. Pour le CNCS-FEUQ, le développement de l'employabilité doit se faire parallèlement ou à la suite de la formation universitaire. Cette dernière doit favoriser l'acquisition de connaissances spécifiques et de compétences générales (travail en équipe, esprit critique, capacités de rédaction, etc.) appropriées pour divers types de débouchés professionnels. En ce qui concerne les compétences particulières qui permettent au diplômé d'être efficace et autonome dans ses futurs emplois, elles varient bien entendu selon les milieux de travail où il souhaite évoluer.

Selon notre analyse des données sur l'insertion, les diplômés sont insérés en emploi lorsqu'ils ont le bagage nécessaire pour pouvoir se stabiliser dans les types d'emploi où ils veulent évoluer. Actuellement, on remarque que le processus d'insertion constitue souvent une période de transition où les diplômés ont l'occasion d'acquérir ce bagage, c'est-àdire de perfectionner leurs connaissances pratiques et leurs compétences particulières, qui ne peuvent toutes être acquises à l'université.

Selon une étude de Krahn et Lowe (1991), menée en 1985 dans trois villes canadiennes, seulement une personne diplômée universitaire sur trois estimait que sa formation universitaire l'avait préparée adéquatement à entrer sur le marché du travail. «[...] les personnes diplômées sont confrontées à des problèmes d'insertion professionnelle: précarité d'emploi, sous-emploi, "inadéquation" de la formation et de l'emploi, disparité des conditions d'insertion selon les champs d'études ${ }^{3} »$.

La période de transition nécessaire entre la formation et l'emploi ralentit considérablement le processus d'insertion et place plusieurs diplômés dans une situation précaire qui les pousse à se maintenir dans des emplois où ils sont surqualifiés et, dans certains cas, qui ne sont pas reliés à leur domaine d'études.

3. C. Trottier, R. Cloutier et L. Laforce, «Typologie de l'insertion professionnelle des personnes diplômées de l'université», dans Revue Canadienne d'enseignement supérieur, vol. XXIV-2, 1996, p. 72. 
Souvent, les compétences particulières et les connaissances pratiques seront acquises à travers l'expérience, ce qui peut se révéler un long processus. Si l'expérience, bien sûr, ne se remplace pas, il est possible de bien se préparer à certains types d'emploi, en parallèle avec sa formation ou à la fin de celle-ci. Pour bien se préparer en fonction de ses intérêts professionnels et pour savoir quelles compétences sont stratégiques à développer, les diplômés devraient notamment pouvoir compter sur les services de placement. Au cours du processus d'orientation et d'exploration des possibilités professionnelles, on doit insister sur l'importance pour le diplômé de se référer aux services de placement pour mettre en œuvre sa stratégie d'insertion. Il faut finalement ajouter que, pour rendre possible l'intervention des services de placement, leur financement devrait être accru significativement.

\section{RECOMMANDATION}

Que les services d'orientation professionnelle et de placement fassent la promotion auprès des étudiants de cycles supérieurs de l'importance d'acquérir plus de polyvalence et, à cet effet, qu'ils offrent des services de consultation stratégique permettant de cibler les compétences et les connaissances les plus appropriées à développer selon la planification professionnelle de l'étudiant.

\section{UNE MEILLEURE CONNAISSANCE DES COMPÉTENCES RECHERCHÉES}

Les étudiants aux cycles supérieurs développent certes des compétences disciplinaires très avancées. Cependant, au moment de trouver un emploi, bien d'autres compétences ont de la valeur aux yeux des employeurs. Le marché du travail a évolué et n'est plus organisé selon un mode taylorien: l'organisation du travail et les tâches à effectuer sont de moins en moins prédéfinies et circonscrites. Les chercheurs d'emploi doivent convaincre leur futur employeur qu'ils possèdent les compétences requises pour évoluer dans un contexte global et mouvant d'organisation du travail.

Pour que les étudiants aux cycles supérieurs puissent cibler les compétences particulières dont ils ont besoin pour améliorer leur employabilité, et pour que les services de placement puissent les aider dans cette tâche, il faut évidemment qu'on possède une excellente information sur les besoins relatifs aux différents types d'emplois, selon les milieux de travail et les secteurs d'activité. Il est primordial de rechercher 
et d'étudier de manière détaillée les compétences et les aptitudes qui permettent aux diplômés de cycles supérieurs de répondre aux exigences du marché du travail. Il ne semble pas exister d'information assez précise et détaillée sur les exigences que commandent les emplois de haut niveau destinés aux diplômés de cycles supérieurs. Néanmoins, des études définissent les compétences générales de plus en plus recherchées sur le marché du travail et dans les différents secteurs. En octobre 1999, un groupe d'experts sur les compétences du Conseil consultatif des sciences et de la technologie du gouvernement du Canada a proposé cinq compétences essentielles dans l'économie du savoir. Il est important de souligner que ces compétences s'avèrent utiles pour œuvrer dans un contexte d'innovation, mais qu'elles ne concernent pas la capacité d'innover des diplômés à proprement parler.

Les services de placement doivent avoir accès à une information détaillée et de qualité sur les compétences et les connaissances pratiques qui sont nécessaires dans les différents emplois spécialisés qu'on peut associer aux diplômés de cycles supérieurs. Pour ce faire, ils devront compter sur des études adéquates sur le sujet. Entre autres choses, il faut se demander s'il ne serait pas nécessaire de mieux évaluer les compétences et aptitudes requises selon les contextes de travail, pour bien appuyer et stimuler le développement et l'innovation. Sur ce dernier aspect, il faut évidemment impliquer les différents partenaires du marché du travail.

\section{RECOMMANDATIONS}

Que les services de placement, afin de pouvoir guider les étudiants, s'assurent de bien comprendre les compétences dont ont besoin les diplômés pour être efficaces et se réaliser en emplois, selon les différents milieux de travail, les types d'emploi et les secteurs d'activité.

Que les partenaires du marché du travail contribuent à mieux définir leurs besoins en termes de compétences et de qualifications, particulièrement pour favoriser le développement et l'innovation, notamment en saisissant la Commission des partenaires du marché du travail de cette problématique. 


\section{UNE OFFRE ACCRUE DE FORMATIONS COURTES POUVANT PRÉPARER LE DIPLÔMÉ À S'INSÉRER PLUS EFFICACEMENT}

\section{La pertinence de l'offre des programmes courts pour le développement de compétences}

En plus d'adapter à certains égards la formation à la recherche, l'université, de même que les collèges, peuvent contribuer à une meilleure stratégie d'insertion des diplômés en mettant à leur disposition davantage de programmes professionnels et de programmes courts. Ces derniers constituent une façon efficace d'acquérir certaines compétences ou connaissances complémentaires en lien avec le projet professionnel. Ces programmes doivent bien sûr s'inscrire dans la mission des universités et offrir la même qualité de formation que les programmes réguliers.

Par ailleurs, il convient de mentionner que plusieurs compétences particulières et connaissances pratiques sont acquises par le biais de formations courtes offertes par les établissements d'enseignement publics et les formateurs privés. L'ensemble des formations courtes disponibles peut permettre de compléter la formation universitaire, mais peut aussi se révéler essentielle pour faciliter la réorientation professionnelle. Le Conseil de la science et de la technologie recommandait en effet de «favoriser la mise sur pied de programmes d'études courts ou concentrés dans le temps, dans les collèges et les universités, afin de réorienter des personnes déjà formées vers des domaines nouveaux en forte demande ${ }^{4} »$.

\section{Rendre les formations courtes plus accessibles}

Bien qu'il existe actuellement un nombre croissant de formations courtes s'adressant aux étudiants, aux personnes en réorientation professionnelle ou aux entreprises devant offrir à leurs employés de la formation continue, leur accessibilité n'est pas toujours adéquate. Pour celui ou celle qui souhaite suivre une formation, la recherche peut être laborieuse. Au Québec, chaque université offre une description de ses programmes courts sur son site Internet, ce qui oblige tous les intéressés à visiter chaque site pour faire ses recherches. Le CNCS-FEUQ privilégie la construction d'un répertoire global des formations courtes offertes. Une

4. Conseil de science et de technologie, Des formations pour une société de l'innovation, avis, Québec, Le Conseil, 1998, p. iv-v. 
brève recherche sur les programmes courts a permis au CNCS-FEUQ de démontrer à la fois le besoin d'établir ce répertoire et l'ampleur du travail que cela représente.

Dans un premier temps, l'établissement d'un répertoire dynamique des programmes courts universitaires demande peu de ressources puisque l'information sur ces programmes est très facilement accessible. Dans une bonne partie des établissements, de tels répertoires existent déjà. Dans la plupart des autres universités n'ayant pas de répertoires distincts pour les programmes courts, le montage de tels répertoires serait une tâche peu exigeante puisque ceux-ci sont intégrés dans les répertoires des programmes et sont facilement identifiables. Seules quelques universités classent les programmes par département ou par faculté, ce qui rend les recherches plus ardues.

Si le ministère de l'Éducation ne dispose pas de répertoire de programmes courts universitaires, il possède cependant des informations sur les programmes de formation professionnelle offerts au secondaire et au collégial. Cette partie du travail serait alors simplifiée.

La difficulté d'un répertoire global serait d'intégrer les formations offertes par les centres de formation privés. Les formations qu'offrent ces centres sont généralement complémentaires à celles des établissements publics et sont d'une grande pertinence en termes de formation pratique. Il y avait au Québec, en date du 17 octobre 2002, quelque 1343 formateurs agréés et 2126 organismes formateurs agréés. Ces formateurs sont régis par le ministère de l'Éducation, qui ne contrôle toutefois pas les formations données. Il conviendrait de compiler les informations quant aux programmes offerts.

Le CNCS-FEUQ croit qu'en matière de formation courte, il faut aussi que le ministère établisse clairement les rôles des établissements publics et des formateurs privés. L'établissement d'un répertoire global serait beaucoup plus bénéfique pour le développement des compétences qui oppose actuellement les différents intervenants en matière d'offre de formation courte. 


\section{RECOMMANDATIONS}

Que les universités, les collèges et les autres établissements d'enseignement assurent, dans la mesure de leurs missions respectives, une offre de programmes courts permettant de répondre aux besoins en développement de compétences et de connaissances.

Que le ministère de l'Éducation établisse clairement le rôle des différents établissements de formation publics et des formateurs privés dans l'offre de formations courtes.

Que le ministère de l'Éducation compile l'information nécessaire sur l'ensemble des formations offertes et qu'il en assure la diffusion pour faciliter les recherches et favoriser l'efficacité d'application de la Loi favorisant le développement de la formation de la main d'œuvre.

\section{LA FORMATION ET LES COMPÉTENCES À DÉVELOPPER LORS DE L'INSERTION}

La formation continue vise d'abord à assurer l'acquisition de compétences et de connaissances devenues nécessaires aux employés dans leur milieu de travail. Le système de développement des compétences et des connaissances constitue un rouage important dans une économie en mouvance et dans des milieux de travail en évolution constante. Or, le Québec avait pris un retard important en termes de formation continue, ce qui a mené à l'élaboration et au dépôt d'une politique sur le sujet. En fait, les chiffres démontrent bien le manque de culture de formation dans les organisations. Selon une étude de Statistique Canada, seulement $11,3 \%$ des entreprises dans le secteur des services en génie, un secteur de haut savoir, ont indiqué offrir de la formation structurée ${ }^{5}$.

En 2001, les ministres de l'Éducation et de l'Emploi, ainsi que la ministre déléguée à l'Emploi, ont présenté une politique de formation continue, dont un des objectifs est d'enraciner une culture de la formation continue chez les entreprises. Dans l'énoncé de cette politique, le gouvernement désire faire face aux insuffisances du système actuel de formation continue, qui n'ont pas été comblées par l'adoption de la Loi favorisant le développement de la formation de la main d'œuvre. Cette politique

5. D. Hamdani, Capacité d'innover, innovation et répercussion: le secteur canadien des services de génie, document de recherche, Statistique Canada, 2001, p. 15. 
s'adresse à l'ensemble de la formation continue et pour l'ensemble des domaines professionnels. Traditionnellement, la formation continue est orientée vers les ordres d'enseignement secondaire et collégial. Or, la formation continue orientée vers des domaines à haute qualification présente des problématiques qui lui sont propres et un examen de la politique qui a été déposée permet de reconnaître son inadéquation aux besoins d'insertion et de formation continue des diplômés de cycles supérieurs.

\section{L'adaptation des compétences lors de l'insertion}

Lors de leur entrée sur le marché du travail, les diplômés de cycles supérieurs sont confrontés à des problèmes liés au fait que leur formation, qui se doit d'être générale, ne répond souvent que partiellement aux exigences de l'emploi qu'ils occupent. Les écarts entre les acquis de formation et les exigences du travail peuvent constituer un frein à une insertion réussie. Ainsi, des mesures doivent être mises en place afin de diminuer ces écarts.

Le volet La formation continue liée à l'emploi de la nouvelle politique de formation continue comporte des éléments qui peuvent améliorer l'adaptation des compétences lors de l'insertion, mais elle n'intègre pas la réalité de la main-d'œuvre issue des cycles supérieurs.

En premier lieu, la mesure visant à mettre au point des outils de veille stratégique et d'écoute des milieux de travail est intéressante, car elle vise entre autres à renforcer les mécanismes de veille du marché du travail, en complétant la formation reçue par le système d'éducation selon les qualifications requises sur le marché du travail. Les diplômés de cycles supérieurs pourraient profiter de ce mécanisme, car un de ces aspects vise le domaine général de l'économie du savoir et des emplois hautement qualifiés. Dans cet aspect particulier de la mesure, la politique met à contribution le ministère de l'Emploi et de la Solidarité sociale et le Comité sectoriel de main-d'œuvre des technologies de l'information et de la communication/Techno-Compétences. Le plan de mise en œuvre de cette politique doit voir à ce que le ministère et le comité sectoriel s'attardent à la formation continue lors de l'insertion des nouveaux diplômés. Lors de leur entrée sur le marché du travail, ces derniers ont besoin de qualifications adaptées à leur nouveau cadre de travail et qui peuvent différer de celles reçues lors de leur formation universitaire. 
Dans le cadre de cette mesure, la nouvelle politique de formation continue propose également de diminuer l'écart entre la formation reçue par le système d'éducation et les besoins de qualification lors de l'entrée sur le marché du travail, en mettant à contribution le ministère de l'Éducation. En effet, le ministère de l'Éducation réalisera des activités de veille stratégique afin d'améliorer la pertinence des programmes d'études en considérant les besoins de qualification du marché du travail. Il sera donc important que cet aspect soit suivi d'un plan d'action, où le ministère de l'Éducation veillera à une plus grande pertinence des programmes d'études, afin de diminuer le besoin d'adaptation des qualifications des nouveaux diplômés entrant sur le marché du travail et, du même coup, les besoins correctifs de l'aspect décrit précédemment.

Pour la formation universitaire, toutefois, une telle adaptation est difficile puisque cette formation n'est généralement pas liée à un type d'emploi défini. Le complément de formation se réalise donc lors de l'insertion professionnelle et, à ce titre, concerne davantage EmploiQuébec. Effectivement, les universités sont maîtres d'œuvre en matière de programme de formation. Le CNCS-FEUQ croit qu'il faut préserver l'autonomie des institutions universitaires. Ainsi, il serait intéressant de doter nos établissements d'observatoires d'insertion professionnelle des diplômés. Nous sommes convaincus que l'étude de l'insertion apportera des éclairages intéressants et alimentera les réflexions de nos établissements. Nous savons qu'une planification stratégique de la formation de la main-d'œuvre, quand elle s'attarde aux champs d'études plutôt qu'aux compétences, s'accompagne souvent de mauvaises décisions. Nous n'avons qu'à nous rappeler le cas de la formation des ingénieurs civils ou, plus récemment, celui de la formation des infirmières.

\section{RECOMMANDATIONS}

Que le ministère de l'Emploi et de la Solidarité sociale prépare un plan d'action élaboré afin de développer des programmes de formation continue dans le domaine de l'économie du savoir et pour les emplois hautement qualifiés.

Que le ministère de l'Éducation, en collaboration avec le ministère de l'Emploi et de la Solidarité sociale et le Fonds québécois de recherche sur la société et la culture, subventionnent la création d'observatoires de l'insertion professionnelle des diplômés de cycles supérieurs. 


\section{La formation continue lors de l'insertion}

Le CNCS-FEUQ croit que les nouveaux diplômés entrant sur le marché du travail doivent faire l'objet d'une attention particulière. Ceux-ci seraient beaucoup plus efficaces s'il leur était donné l'opportunité de recourir à de la formation permettant, au besoin, de compléter leurs connaissances et leurs compétences générales au moment de leur entrée dans un nouveau type d'emploi. Dans plusieurs cas, les responsabilités dont ils ont la charge requièrent non seulement un niveau élevé de connaissances, mais aussi un champ étendu de compétences de même qu'une grande polyvalence.

Malheureusement, la politique gouvernementale proposée ne prévoit aucune mesure visant à favoriser la formation continue lors de l'insertion des étudiants de cycles supérieurs. En fait, cette politique a fait le choix, et à juste titre, de permettre à la main-d'œuvre la moins qualifiée ou la moins reconnue d'acquérir des compétences par l'emploi: elle vise à qualifier $100 \%$ de la population québécoise. Par ailleurs, si cet objectif constitue un investissement pour le Québec, la formation lors de l'insertion des étudiants de cycles supérieurs représente pour sa part un moyen de faire fructifier les investissements en formation avancée. Une insertion optimale permettrait d'accroître nettement l'efficacité dans les milieux de travail.

\section{RECOMMANDATION}

Que le ministère de l'Emploi et de la Solidarité sociale, en collaboration avec le ministère du Développement économique, de l'Innovation et de l'Exportation, mette en place des programmes favorisant la formation continue dès l'entrée des diplômés sur le marché du travail.

\section{La formation continue dans les petites entreprises et chez les travailleurs autonomes}

Faire reconnaître l'importance d'une telle approche de formation qui vise à optimiser l'insertion et ses retombées n'est pas une tâche facile. Les grandes entreprises possèdent déjà certaines politiques à cet égard, mais l'évolution des pratiques dans la majorité des milieux doit être soutenue par le gouvernement, en particulier auprès des petites organisations et des travailleurs autonomes. 
La politique de la formation continue s'attarde à la problématique du manque d'implantation de la culture de la formation continue dans les entreprises et chez les travailleurs autonomes et atypiques. En effet, à l'automne 2002, Emploi-Québec et la Commission des partenaires du marché du travail ont réalisé une étude sur les besoins en formation chez ces entreprises et travailleurs dont les activités de formation continue sont insuffisantes. Les conclusions de cette étude seront intégrées à la future stratégie gouvernementale d'intervention en matière de ressources humaines qui sera adoptée par le ministre d'État aux Ressources humaines et au Travail.

Le CNCS-FEUQ considère que la future stratégie gouvernementale en matière de ressources humaines devra mettre en place des mesures visant à aider les petites entreprises à offrir de la formation continue à leurs employés et à permettre aux travailleurs autonomes de suivre des formations en vue d'améliorer leurs compétences, ce qui vaut également pour les travailleurs œuvrant dans les secteurs de l'économie du savoir et de l'innovation, de même que dans d'autres secteurs d'emploi nécessitant une formation des cycles supérieurs.

\section{RECOMMANDATION}

Que la stratégie gouvernementale adoptée par le ministre d'État aux Ressources humaines et au Travail mette en place des mesures visant à offrir de la formation continue pour les petites entreprises et les travailleurs autonomes en incluant les secteurs de l'économie du savoir et de l'innovation.

\section{Les aspects qualifiants et la transférabilité de la formation continue}

Le Conseil de la science et de la technologie observait que «même dans les entreprises les plus actives en formation continue, on cherche de plus en plus à mettre l'accent surtout sur la formation nécessaire à l'atteinte immédiate de leurs objectifs à court terme ${ }^{6}{ }$. Pour le CNCS-FEUQ, cela signifie que peu d'intérêt est porté au développement de compétences relatives à l'innovation dans les milieux de travail. La politique de formation continue propose quelques mesures visant à inciter les entreprises à offrir des programmes de formation continue qualifiants et dont les

6. Conseil de science et de technologie, Des formations pour une société de l'innovation, op. cit. note 80 , p. 19. 
compétences sont transférables. Cependant, ces mesures ne tiennent pas compte de la spécificité des besoins de main-d'œuvre agissant dans les secteurs de l'innovation et de l'économie du savoir. De surcroît, l'approche s'applique difficilement au type d'emplois qu'occupent les diplômés issus de programmes de formation des cycles supérieurs.

La politique de formation continue met notamment à la disposition des comités sectoriels des outils de diverses natures: création de normes professionnelles à partir du cadre général de développement et de reconnaissance des compétences, certification professionnelle, crédit d'impôt aux entreprises, programme d'apprentissage en milieu de travail, etc. Ces mesures ne s'adressent pas à la main-d'œuvre innovante issue des cycles supérieurs.

Toutefois, la Commission des partenaires du marché du travail entend élargir le cadre général des apprentissages qui se réalisent en milieu de travail à d'autres voies que les seuls programmes d'apprentissage, il faudrait étudier les compétences requises par la main-d'œuvre pour le développement des secteurs de l'économie du savoir et de l'innovation. Cette étude pourra nous éclairer sur les problématiques spécifiques de formation de la main-d'œuvre dans ces secteurs et mener, au besoin, à la création d'un comité sectoriel qui pourrait voir à l'élaboration d'outils plus appropriés.

\section{RECOMMANDATIONS}

Que la Commission des partenaires du marché du travail étudie les compétences requises par la main-d'œuvre pour le développement des secteurs de l'économie du savoir et de l'innovation.

Que la Commission des partenaires du marché du travail explore la possibilité de créer un comité sectoriel de main-d'œuvre propre au secteur de l'économie du savoir et de l'innovation.

Que le ministère de l'Éducation favorise une culture de la formation continue qui commence au moment de l'insertion sur le marché du travail et qui se poursuit selon les exigences de l'innovation et du développement.

Que cette culture de la formation continue soit soutenue par des mesures incitatives ou de soutien auprès des personnes et entreprises chez qui sa promotion est difficile, notamment les petites organisations et les travailleurs autonomes. 


\section{La formation continue et l'insertion organisées et financées par des organismes collecteurs}

Les PME constituent le plus important créateur d'emploi au Québec, un terrain fertile à l'innovation et un milieu d'insertion professionnelle important des diplômés. Plusieurs de ces entreprises ont plus de 250000 \$ de masse salariale et sont assujetties à la Loi favorisant le développement de la formation de la main-d'œuvre (loi 90). Dans un tel contexte, notamment à cause des multiples formulaires à remplir, plusieurs PME préfèrent envoyer au gouvernement le $1 \%$ exigé et ne pas se préoccuper de planifier la formation de leur personnel. Cette situation, bien qu'explicable, nous paraît déplorable. Pour y remédier et financer les besoins de formation et d'insertion des diplômés de cycles supérieurs, nous recommandons à ces entreprises de se regrouper en fonction de leurs intérêts et secteurs d'emploi et de créer des organismes collecteurs. Ainsi, plutôt que de garnir le Fonds national de formation de la main-d'œuvre, les sommes non dépensées seraient amassées au sein d'un organisme qui assurerait le développement de programmes d'insertion et la mise en réseau des entreprises innovantes.

\section{RECOMMANDATION}

Que les entreprises innovantes et des milieux de l'économie du savoir, assujetties à la Loi favorisant le développement de la formation de la main-d'œuvre, se regroupent en organisme collecteur afin d'instituer des mécanismes de formation continue et d'insertion professionnelle des diplômés de cycles supérieurs. 


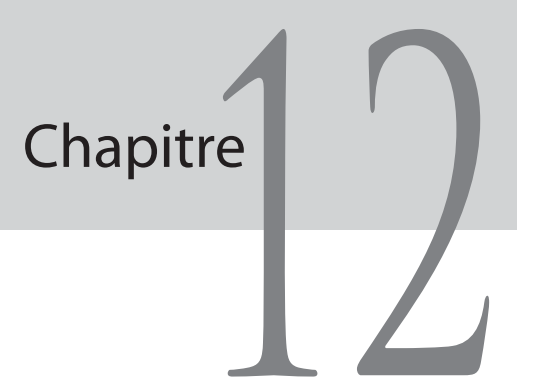

\section{LA CAPACITÉ D'INNOVER ET LA MISE EN VALEUR DES DIPLÔMÉS}

La mise en valeur du potentiel du diplômé de cycles supérieurs repose d'abord sur la structure des organisations où il évolue, sur leur capacité d'innover et sur le soutien que le diplômé peut y trouver. Pour les organisations, le diplômé de cycles supérieurs représente en fait un potentiel de développement et d'innovation qu'il faut valoriser. Or $26 \%$ des diplômés de maîtrise considèrent que leurs connaissances sont mal utilisées par leur employeur ${ }^{1}$. En outre, une enquête menée à l'Université Laval montre que très peu de diplômés $(11,3 \%)$ mettent en œuvre, dans le cadre de leur emploi, les compétences et habiletés reliées à l'innovation.

1. M. Audet, Les stratégies d'insertion professionnelle des titulaires d'un baccalauréat ou d'une maîtrise à la lumière de leur expérience du marché du travail, MEQ, Direction de l'enseignement et de la recherche universitaire, Québec, 1999, p. 81. 
POUR:

Que le diplômé de cycles supérieurs puisse contribuer au développement et à l'innovation par la mise en valeur de son plein potentiel.

\section{ON DEVRAIT INTERVENIR SUR:}

Les moyens d'accroître la capacité d'innover des organisations et la capacité des diplômés de cycles supérieurs à constituer une force d'innovation dans ces milieux.

\section{UNE MEILLEURE CONNAISSANCE DES COMPÉTENCES DES DIPLÔMÉS DE CYCLES SUPÉRIEURS}

Une question se pose principalement à propos de l'embauche et de l'exploitation du potentiel des diplômés de cycles supérieurs : les entreprises sont-elles conscientes des compétences développées par ces diplômés et savent-elles ce qu'ils peuvent leur apporter?

En effet, tout en se spécialisant dans leur domaine de recherche, les étudiants aux cycles supérieurs acquièrent plusieurs compétences générales essentielles à la réalisation de leur projet et qui sont, de plus, entièrement transférables. Le contexte de formation dans lequel ces étudiants évoluent leur permet de développer leur capacité de recherche, de rédaction, de synthèse et d'analyse tout en développant leur esprit critique. Ces habiletés peuvent apporter beaucoup aux entreprises dans une société d'innovation, si on met en valeur les diplômés qui les possèdent. Par exemple, les capacités que développent les étudiants de cycles supérieurs leur permettent d'effectuer des recherches pour tenir informé leur employeur sur les nouvelles technologies ou méthodes d'intervention récemment mises au point. Elles accentuent alors la capacité d'innover d'une entreprise en favorisant l'intégration de mécanismes de veille technologique fort utiles à l'organisation. Il y a donc nécessité de mieux faire connaitre les compétences acquises par les diplômés de cycles supérieurs. 


\section{RECOMMANDATIONS}

Qu'on fasse mieux connaître les compétences générales acquises par le diplômé de cycles supérieurs auprès de leurs employeurs potentiels.

Que les services de placement universitaire produisent, à l'intention des employeurs potentiels, des dépliants expliquant les compétences générales développées par les diplômés de cycles supérieurs et ce qu'ils peuvent apporter à leurs organisations.

\section{L'INSERTION DE DIPLÔMÉS DE CYCLES SUPÉRIEURS POUR ACCROÎTRE LA CAPACITÉ D'INNOVER}

Selon une étude du Comité consultatif des sciences et de la technologie, l'offre de compétences n'accroît pas forcément la demande ${ }^{2}$, ce qui signifie que la formation et le développement des compétences ne suffit pas à développer une culture d'innovation dans les organisations. Certes il faut intégrer des diplômés de cycles supérieurs dans les milieux de travail, mais pour qu'ils puissent mettre à profit leurs compétences en recherche et en innovation, il faut idéalement qu'ils se trouvent dans un milieu permettant l'utilisation de ces compétences et qu'ils aient la possibilité de suivre une formation pratique appropriée. Si certains milieux offrent à cet égard un encadrement intéressant, plusieurs autres ne sont pas nécessairement organisés et expérimentés en termes de recherche, de développement et d'innovation. Dans ce dernier cas, les organisations ne savent pas exactement ce qu'elles cherchent et de quelle manière procéder. C'est ce qui se produit lors de la création de nouveaux postes: des entreprises ont recours aux services de placement universitaires pour cerner le profil des candidats qu'ils recherchent.

En fait, les barrières à l'innovation varient d'un milieu à l'autre. Les diplômés de cycles supérieurs, par leur formation, peuvent favoriser le développement et linnovation dans plusieurs organisations, dont les PME, les coopératives, les organismes communautaires, les organismes gouvernementaux et les OSBL. Le CNCS-FEUQ veut ici préciser que quatre aspects doivent être considérés pour favoriser l'innovation

2. G. Lowe et K. McMullen, Obstacles et incitatifs à la formation, sommaire, Comité consultatif des sciences et de la technologie, Groupe d'experts sur les compétences, Canada, 1999, 5 p. 
dans certains milieux non innovants: la promotion de l'importance de la recherche et de l'innovation, l'insertion de diplômés de cycles supérieurs bien formés, le développement et le soutien de l'expertise dans les organisations et le développement d'un secteur de recherche et de développement périphérique aux organisations pour répondre aux besoins de plusieurs d'entre elles.

\section{Les milieux d'innovation sociale: OSBL, milieux communautaires et COOP ${ }^{3}$}

Le processus de développement des entreprises d'économie sociale, des organismes communautaires et des OSBL se fonde généralement sur une expérimentation pratique ou une recherche de type empirique ou pratique. En effet, on dit parfois de ces organismes qu'ils sont des laboratoires sociaux, des lieux d'innovation sociale, économique et culturelle. Toutefois, ces organisations ont rarement le temps et la possibilité de recourir à des ressources spécialisées pour étudier plus en profondeur leurs problématiques ou leurs méthodes d'intervention actuelles ou potentielles.

Pourtant, la structure des OSBL et des coopératives est propice au partage rapide et efficace du savoir, condition essentielle à l'innovation. «Pour qu'un organisme puisse partager le savoir de façon rapide et efficace, il est bon que sa structure soit horizontale, ouverte, interdisciplinaire et configurée en fonction de l'étude d'un problème ${ }^{4}$.» Les milieux d'innovation sociale répondent en général à cette description et peuvent, de surcroît, compter sur des diplômés de sciences sociales qui font preuve d'une grande polyvalence. Comme le relate un rapport de la Commission universitaire sur les programmes, «selon le Conseil supérieur de l'éducation, la formation acquise par les diplômés en sciences sociales rend ceux-ci remarquablement polyvalents et particulièrement habiles à exécuter de nombreuses tâches, à assumer plus de responsabilités». Cette condition est importante pour favoriser le développement et l'innovation dans des organismes relativement modestes dont les ressources sont très limitées. Cependant, il est rare que le personnel

3. La majorité de cette section est tirée de: CNCS-FEUQ, Politique scientifique: pour une vue d'ensemble, avis, Fédération étudiante universitaire du Québec, 2000, 32 p.

4. Statistique Canada, «Forum de haut niveau de l'OCDE sur la gestion du savoir - un nouveau défi pour les entreprises et les organismes », dans Bulletin de l'analyse en innovation, vol. 3, $\mathrm{n}^{\circ} 1$, Canada, février 2001, p. 8. 
dans ces organismes soit formé pour la recherche et le développement ou puisse développer concrètement ces compétences. De plus, il leur est difficile de consacrer du temps à ces activités, étant donné le manque de ressources chronique des organismes où ils évoluent.

En certaines occasions, des regroupements d'organisations d'économie sociale ou communautaires ont l'occasion d'établir des partenariats de recherche avec les universités. C'est ce qui est visé par les Alliances de recherche universités-communautés (ARUC), qui sont soutenues par le Conseil de recherche en sciences humaines du Canada (CRSH). Le programme a pour objectif d'appuyer la création d'alliances spéciales entre les universités et les communautés qui, par un processus axé sur la collaboration continue et l'apprentissage mutuel:

- favorisent la recherche novatrice, la formation et l'avancement des connaissances dans des domaines d'importance pour le développement social, culturel ou économique des communautés ;

- favorisent l'échange de connaissances, de ressources et de compétences entre les universités et les organismes de la communauté;

- enrichissent la recherche, les méthodes d'enseignement et les programmes des universités ;

- renforcent la capacité des collectivités de prendre des décisions et de résoudre des problèmes;

- rehaussent la formation et l'employabilité des étudiants en leur donnant diverses possibilités d'acquérir des connaissances, des compétences et des qualités professionnelles grâce à des travaux de recherche pratiques et à une expérience connexe ${ }^{5}$.

Dans une autre optique, le Centre interdisciplinaire de recherche et d'information sur les entreprises collectives (CIRIEC) propose aussi différentes activités pertinentes. Celles-ci visent à améliorer les capacités d'innovation générales des organismes sans but lucratif qui œuvrent dans le domaine de l'économie sociale, plutôt quà améliorer celles-ci sur une base individuelle, par le ciblage d'associations particulières. L'action du CIRIEC n'apporte pas directement une amélioration de la capacité

5. Conseil de recherches en sciences humaines du Canada, Alliances de recherche universitéscommunautés (ARUC), site Internet, août 2002, <http://www.crsh.ca/francais/renseignements/guidesubventions/aruc.html>. 
d'innovation des organismes sans but lucratif par l'apport de diplômés qui augmenteront leurs capacités de recherche, mais vise plutôt à accroître les connaissances des organismes qui choisissent d'en être membres sur les façons d'améliorer leurs capacités d'innovation.

Ces initiatives présentent l'avantage de promouvoir la culture d'innovation dans les organismes participants et de favoriser les développements de champs de recherche d'intérêt pour l'innovation sociale. De plus, elles permettent l'insertion temporaire de chercheurs et la formation adaptée de diplômés ayant une expérience de la recherche et du développement dans ces milieux.

Toutefois, ces partenariats sont relativement exigeants pour les deux parties et ne permettent pas de consolider l'expertise. Les OSBL et les organismes d'économie sociale ont de la difficulté à conserver un personnel pouvant se consacrer à l'aspect recherche, à définir les besoins et les axes possibles de recherche de manière concrète. Aussi, les chercheurs universitaires doivent souvent faire de nombreuses démarches afin de connaître ces organisations, les problématiques auxquelles elles sont confrontées et les milieux dans lesquels elles évoluent. Le processus peut être long et laborieux, mais il est préalable à l'élaboration de tout projet de recherche en collaboration.

Le Programme de contributions pour les organismes sans but lucratif de consommateurs et de bénévoles du Bureau de la consommation constitue un exemple intéressant à considérer afin d'accroître la capacité d'innover des organismes à but non lucratif et de développer leurs capacités de recherche dans des domaines d'application concrète. Ce programme finance des projets de recherche dans les OSBL, leur offrant ainsi des ressources pour améliorer et développer leurs capacités de recherche. Il contribue alors au développement, à la stabilisation et à la consolidation de leur expertise. La complexité des sujets traités demande aux organismes d'intégrer des diplômés de cycles supérieurs spécialisés dans plusieurs domaines.

Ces diplômés peuvent ajouter une plus-value aux activités des organismes à but non lucratif en réalisant des recherches qui contribuent de manière significative au processus décisionnel du gouvernement canadien en matière de consommation. Les organismes à but non lucratif voient de plus tomber une barrière importante à la réalisation de la recherche, soit 
le manque de financement. En somme, ils peuvent acquérir une expertise qui leur permettra d'apporter des solutions encore plus novatrices dans l'avenir.

La présence de personnel scientifique de recherche dans certaines organisations communautaires et d'économie sociale semble également salutaire pour faciliter l'existence d'une collaboration efficace avec le milieu scientifique, et le déploiement de l'innovation sociale. Il joue en outre un rôle essentiel pour le transfert des connaissances et l'utilisation, pour le compte des organisations, des études déjà publiées. En effet, un personnel scientifique de recherche et de développement au service des organisations permet d'adapter les recherches à leurs besoins spécifiques. Ce personnel peut être en lien avec un seul organisme, des regroupements d'organismes ou dépendre d'organisations ou de centres de transfert offrant des services aux organismes, mais sa mise en réseau doit faire l'objet d'une attention particulière.

On pourrait par exemple confier cinq tâches primordiales au personnel scientifique de recherche:

- l'identification des besoins de recherche;

- le soutien scientifique au développement;

- le maintien d'interactions avec le milieu scientifique;

- la promotion et la diffusion des pratiques et innovations;

- et le soutien à la formation et à l'amélioration de la culture organisationnelle.

L'existence d'un tel réseau scientifique rend possible une foule d'initiatives de transfert qu'il faudra aussi explorer.

\section{RECOMMANDATIONS}

Que les ministères provinciaux chargés de stimuler le développement et l'innovation favorisent l'insertion de diplômés de cycles supérieurs dans les OSBL, les milieux communautaires et ceux de l'économie sociale qui accusent un retard en termes de développement et d'innovation.

Que le gouvernement du Canada transfère au Québec les fonds affectés à la Stratégie Emploi Jeunesse afin d'assurer une cohérence dans l'offre de services et de respecter l'entente sur la main-d'œuvre entre les gouvernements. 
Qu'Industrie Canada accroisse le financement du CRSH afin de multiplier les Alliances de recherche universités-communautés (ARUC).

Que des programmes de soutien permettant de financer les travaux de recherche et le développement d'expertise soient élargis pour consolider les effectifs des organisations communautaires, sociales et sans but lucratif.

Que les ministères provinciaux et Industrie Canada, considérant que les OSBL, les milieux communautaires et ceux de l'économie sociale offrent des contextes propices à l'innovation sociale, soutiennent fortement la consolidation des effectifs de développement dans ces milieux et leur mise en réseau.

\section{Les PME et le secteur périphérique de R-D}

Les entreprises qui concentrent davantage de connaissances innovent généralement plus que les autres. Ce faisant, les entreprises innovatrices engendrent une activité économique importante qui se répercute sur la croissance de l'emploi.

De ce fait, la mise en valeur des compétences des diplômés de maîtrise devrait être considérée comme un enjeu majeur. En stimulant le développement et l'innovation, en plus de favoriser une certaine activité, on diminue la surqualification des diplômés de cycles supérieurs et, incidemment, celle de tous les autres. Ces retombées de l'innovation pourraient être beaucoup plus exploitées par les petites entreprises. En effet, si les données indiquent généralement que les moyennes et les grosses entreprises sont plus enclines à innover que les petites, il existe des moyens de favoriser l'innovation dans ces dernières. Pour les petites entreprises, mais aussi pour plusieurs autres, la faible concentration de connaissances constitue parfois un frein à l'innovation. La relation entre le taux d'innovation et la concentration de connaissances a d'ailleurs été démontrée ${ }^{6}$.

Or, s'il n'est pas toujours possible pour les entreprises d'employer tout le personnel dont elles ont besoin pour favoriser l'innovation, plusieurs arrivent tout de même à atteindre des taux d'innovation intéressants. Selon Statistique Canada, ces entreprises arrivent à recruter à l'externe les spécialistes dont elles ont besoin ${ }^{7}$. En France, des études révèlent

\footnotetext{
6. Statistique Canada, «Connaissances et innovation: mettre à profit les meilleures compétences », dans Bulletin de l'analyse en innovation, vol. 3, no 1, Canada, février 2001, p. 6.

7. Idem.
} 
également qu'un secteur de recherche se développe en périphérie des entreprises, incluant des associations techniques et des sociétés offrant des ressources hautement qualifiées. Ainsi, certaines entreprises préfèrent traiter avec ce secteur tampon plutôt que d'engager directement des scientifiques ${ }^{8}$.

En conséquence, les étudiants de cycles supérieurs peuvent, à défaut d'être employés dans les entreprises elles-mêmes, travailler pour des firmes ou des consultants qui leur offrent des services spécialisés en matière de développement et d'innovation. De plus, l'établissement de partenariats de recherche précompétitive peut dans certains cas s'avérer intéressante pour soutenir des efforts de recherche communs à plusieurs entreprises. Â cet effet, le CNCS-FEUQ désire rappeler que le développement d'une culture d'innovation et d'une expertise en R-D en entreprise doit demeurer un objectif prioritaire.

\section{RECOMMANDATION}

Que le MFER, Industrie Canada et Développement des ressources humaines Canada soutiennent les initiatives de développement et d'innovation dans les PME et les organisations de taille moyenne et petite, soit par des incitatifs à l'insertion de diplômés de cycles supérieurs, soit par des mesures encourageant le recours à des services spécialisés pour appuyer l'innovation.

\section{LE SOUTIEN DES EFFORTS DES DIPLÔMÉS DE CYCLES SUPÉRIEURS POUR FAVORISER L'INNOVATION}

Il est clair que la présence de diplômés de cycles supérieurs peut contribuer à accroître la concentration de connaissances dans une entreprise et, conséquemment, à favoriser l'innovation. Cependant, le CNCS-FEUQ est très conscient que la dynamique de l'innovation est complexe et qu'elle ne peut pas reposer sur les seuls diplômés de cycles supérieurs. Toutefois, des diplômés ayant développé les compétences pratiques pertinentes viendraient accroître les retombées de leur insertion. Or ce développement de compétences reste peu fréquent et souvent fragmentaire.

8. J.-J. Paul et C. Perret, Caractéristiques des équipes de recherche et insertion professionnelle des docteurs scientifiques, rapport préparé pour la Direction générale de la recherche et de la technologie, IREDU/CÉREQ, Dijon, France, 1997, 124 p. 
De plus, le CNCS-FEUQ croit fermement qu'agir sur la formation et l'acquisition de compétences aux cycles supérieurs ne suffit pas, dans plusieurs milieux de travail, à favoriser l'insertion optimale. Il existe des entreprises qui n'innovent pas, comme nous l'avons mentionné plus haut. «Selon Mintzberg (1979), au fur et à mesure qu'une organisation innovatrice avance en âge, de nombreuses forces entrent en action et la poussent vers la bureaucratisation et l'uniformisation, ce qui peut convenir aux employés qui vieillissent eux aussi et accueillent favorablement la stabilité $^{9}$.»

Les données présentées par Statistique Canada tendent à prouver cette analyse. "Les entreprises qui avaient connu un changement de propriétaire ces dernières années avaient un taux d'innovation plus élevé. Des entreprises sont acquises parce que leurs possibilités technologiques vont bien au-delà de l'utilisation qui en est faite par les gestionnaires en place $^{10}$.»En somme, les entreprises vieillissantes peuvent être résistantes à l'innovation. En fait, il s'agit probablement d'un des éléments importants expliquant le fort pourcentage d'entreprises qui n'innovent pas.

Pour le CNCS-FEUQ, l'insertion de diplômés de cycles supérieurs constitue certainement un élément à considérer pour conserver ou relancer la culture d'innovation d'une entreprise. Un pourcentage croissant de jeunes diplômés de cycles supérieurs se lancent en affaires chaque année ou effectue du travail autonome. Le CNCS-FEUQ croit qu'une entreprise déjà solide peut aussi servir de noyau pour les diplômés de cycles supérieurs: c'est ce qu'on pourrait appeler l'«entrepreneuriat interne». En d'autres termes, il existe des moyens de mettre de l'avant des projets ou des idées de développement à l'intérieur d'une entreprise existante, si on peut y faire exister les conditions de l'innovation. La formule exige une certaine autonomie de la part des «entrepreneurs internes» et une certaine aide financière. Cette avenue réduit considérablement les risques d'avortement ou de faillite que courent les nouveaux entrepreneurs, et qu'on impute le plus souvent à des carences en gestion. De plus, l'«entrepreneuriat interne» pourrait réduire la volatilité des entreprises dans certains secteurs.

\footnotetext{
9. D. Hamdani, Capacité d'innover, innovation et répercussion: le secteur canadien des services de génie, document de recherche, Statistique Canada, 2001, p. 26.

10. Ibid., p. 25.
} 
Actuellement, il existe de trop rares programmes d'insertion de diplômés universitaires visant le développement des entreprises. Il convient de mettre de l'avant des programmes suffisants d'insertion de diplômés de cycles supérieurs dans des postes relatifs à l'innovation. Toutefois, il faut considérer sérieusement les difficultés auxquelles se butent les diplômés une fois en entreprise. Pour que le diplômé puisse tirer son épingle du jeu dans une entreprise qui a peu de culture d'innovation, il faut indéniablement qu'il soit soutenu quant aux aspects du travail de développement et d'innovation qu'il connaît insuffisamment. Souvent, les principales difficultés de l'innovation touche les plans économiques et ceux de la mise en marché, comme l'indiquent plusieurs études de Statistique Canada. Le tableau 12.1 souligne clairement cette prédominance du facteur économique comme entrave à l'innovation.

TABLEAU 12.1

\section{Entraves à l'innovation}

\begin{tabular}{|l|c|c|}
\hline \multirow{2}{*}{} & $\begin{array}{c}\text { Secteur des services } \\
\text { de génie }\end{array}$ & $\begin{array}{c}\text { Secteur des services aux } \\
\text { entreprises }\end{array}$ \\
\cline { 2 - 3 } & $\begin{array}{c}\text { Entreprises innovatrices } \\
\text { déclarant que ces facteurs } \\
\text { sont très importants ou } \\
\text { primordiaux (\%) }\end{array}$ & \\
\hline Risque élevé lié à la faisabilité de projets dinnovation & 34,6 & \\
\hline Risque élevé concernant la réussite sur le marché & 34,3 & 33,6 \\
\hline Innovation facilement copiée par d'autres entreprises & 30,1 & 38,6 \\
\hline Difficulté de prédire les coûts de línnovation & 39,7 & 31,3 \\
\hline Coûts élevés & 43,7 & 36,2 \\
\hline Longue période d'amortissement & 32,8 & 44,2 \\
\hline Manque de fonds propres & 37,2 & 31,4 \\
\hline Manque de capitaux extérieurs & 35,5 & 40,8 \\
\hline Manque de personnel qualifié & 26,7 & 37,5 \\
\hline Manque d'équipements & 13,7 & 29,2 \\
\hline Résistance interne & 5,8 & 15,6 \\
\hline Long processus administratif d'approbation & 7,1 & 6,9 \\
\hline $\begin{array}{l}\text { Lois et règlements ayant une incidence sur les projets } \\
\text { d'innovation }\end{array}$ & 9,8 & 6,3 \\
\hline
\end{tabular}

* Groupe de recherche institutionnel sur les effectifs étudiants, L'insertion professionnelle des personnes diplômés de 1993, Relance 1996, document n 4, Université Laval, Québec, avril 1997, $188 \mathrm{p}$. 
Pour les diplômés de cycles supérieurs qui veulent stimuler l'innovation, certaines qualités complémentaires sont nécessaires: la capacité à introduire et à promouvoir des nouveaux produits et procédés, à convaincre souvent les utilisateurs de modifier leurs habitudes, à changer les façons de faire, etc. Pour le CNCS-FEUQ, les programmes d'insertion de diplômés de cycles supérieurs dans des emplois en innovation doivent être accompagnés de programme de soutien et d'accès à des réseaux d'expertise et de formation. Ainsi, le diplômé peut forger sa place dans une entreprise en la développant, ce qui devient une forme d' "entrepreneuriat interne » et permet d'intégrer une certaine culture d'innovation et de renouvellement dans l'entreprise.

Afin de mettre de l'avant à large échelle ce type d'insertion et de soutien, le gouvernement du Canada devrait tenter l'expérience dans le secteur technologique avec le Programme PARI et le Réseau canadien des technologies. Ces initiatives du Conseil national de recherche apparaissent tout à fait appropriées pour soutenir l'insertion de diplômés de cycles supérieurs, l'«entrepreneuriat interne» et l'acquisition de compétences nécessaires à l'innovation. Le Programme PARI devrait élargir ses programmes de soutien à l'embauche et, surtout, y adjoindre des ressources pour venir en aide aux nouveaux employés. À cette fin, le Réseau canadien des technologies apparaît une structure intéressante.

\section{RECOMMANDATIONS}

Que les ministères provinciaux concernés soutiennent un "réseautage" et une mise en commun des efforts de recherche et de développement des PME, notamment en ce qui concerne la recherche précompétitive.

Que le Programme PARI favorise l'insertion de diplômés de cycles supérieurs pour le développement et l'innovation dans les organisations et offre des possibilités de formation, un réseau de référence et un appui réel pour que les employés orientent leurs actions de la bonne façon et favorisent vraiment l'innovation.

Que les ministères responsables étudient les moyens concrets qui peuvent être les plus efficaces pour encourager l'« entrepreneuriat interne ».

Que les ministères responsables de stimuler le développement et l'innovation, comprenant l'importance de diverses compétences particulières, dont celles liées à la promotion des projets, à leur mise en marché ou à leur réalisation, soutiennent l'acquisition de ces compétences chez les diplômés à leur entrée sur le marché du travail. 


\section{NOTICES BIOGRAPHIQUES}

Stéphanie Andrews est doctorante au $\mathrm{Ph}$. D. en économique à l'Université McGill. Sa thèse porte entre autres sur le phénomène de l'émigration des infirmières canadiennes vers les États-Unis au cours du $\mathrm{xx}^{\mathrm{e}}$ siècle. Elle tente d'évaluer dans quelle mesure la tendance récente de l'émigration de ce groupe de travailleuses se démarque réellement de la tendance lourde de long terme.

Louise Dandurand, Ph. D. en sciences politiques de l'Université de Toronto, est présidente-directrice générale du Fonds québécois de la recherche sur la société et la culture. Elle a publié plusieurs articles dans les domaines de la politique scientifique et de l'histoire des sciences. De 1988 à 1997, elle a été secrétaire générale du Conseil de recherche en sciences humaines du Canada et, à ce titre, responsable de la politique, de la planification, de l'évaluation, des relations internationales et des relations publiques, entre autres. Au cours de la même période, elle a été présidente par intérim et directrice générale des programmes au CRSH, participant ainsi au développement de différents programmes. Elle a également été secrétaire générale du Conseil des arts du Canada. En janvier 1997, elle a été nommée vice-rectrice à la planification stratégique et financière et en août 1998, vice-rectrice à la recherche, à la création et à la planification de l'Université du Québec à Montréal. 
Sylvie Dillard, titulaire d'une maîtrise en économie de l'Université Laval, est présidente-directrice générale du Fonds québécois de la recherche sur la nature et les technologies. Cet organisme, créé en juin 2001 par le gouvernement du Québec, en remplacement du Fonds pour la formation de chercheurs et l'aide à la recherche, a pour mandat d'appuyer le développement de la formation de chercheurs et de la recherche, et leur contribution à l'innovation, dans les domaines des sciences naturelles, des sciences mathématiques et du génie.

Madame Dillard a occupé auparavant plusieurs postes dans la haute administration publique québécoise, en lien avec le développement scientifique et technologique du Québec. Ainsi, de 1992 à 1995, elle était directrice générale des politiques au ministère de l'Industrie, du Commerce, de la Science et de la Technologie. Elle a notamment contribué à la mise en place des Sociétés de capital de risque Innovatech Chaudière-Appalaches et Sud du Québec, au lancement du Fonds de l'autoroute de l'information, ainsi qu'à l'actualisation de la stratégie industrielle du gouvernement (stratégie des Grappes industrielles). De 1995 à 1998, elle était sousministre adjointe à la planification, à la recherche et à l'évaluation au ministère de la Santé et des Services sociaux et a notamment contribué à la consolidation des Centres hospitaliers universitaires et au développement d'Instituts universitaires de recherche, en collaboration avec le Fonds de recherche en santé du Québec et le Conseil québécois de la recherche sociale. Elle a également lancé le plan d'action du MSSS relatif à l'éthique en recherche.

De 1998 à juin 2001, elle était présidente-directrice générale du Fonds pour la formation de chercheurs et l'aide à la recherche, un organisme du gouvernement du Québec ayant pour mission de soutenir la recherche et la formation par la recherche dans les institutions post-secondaires et ce, dans l'ensemble des domaines scientifiques. Son organisation ayant été rattachée au ministère de la Recherche, de la Science et de la Technologie dès la fin de 1998, elle a collaboré étroitement aux travaux de la nouvelle Politique québécoise de la science et de l'innovation.

Elle a siégé sur de nombreux conseils d'administration d'organismes voués au développement et au transfert de la recherche. Elle est aujourd'hui membre des conseils d'administration de Valorisation-Recherche Québec et de plusieurs des consortiums de recherche en sciences et en génie mis en place par cette société. Elle siège également sur les conseils d'administration de Génome Québec, de la Fondation canadienne de recherche sur 
les services de santé, de la Société pour la promotion de la Science et de la Technologie, ainsi que de la Commission consultative de l'Institut des matériaux industriels. Elle a été nommée en octobre 2002 membre du Conseil d'administration de la Caisse de dépôt et placement du Québec.

Brigitte Gemme est agente de recherche au Centre interuniversitaire de recherche sur la science et la technologie (CIRST) et enseigne la méthodologie de recherche en science, technologie et société (STS) à l'Université du Québec à Montréal. Elle détient une maîtrise en sociologie et un baccalauréat en STS. Elle coordonne le projet de recherche «La formation étudiante aux cycles supérieurs à l'ère des collaborations université-milieu».

Yves Gingras est professeur au Département d'histoire de l'Université du Québec à Montréal (UQAM) et titulaire de la Chaire de recherche du Canada en histoire et sociologie des sciences. Ses travaux portent sur l'histoire et la sociologie des sciences et de l'enseignement supérieur. Il a publié "What Did mathematics Do to Physics?», History of Science, vol. 39, 2001, p. 383-416, « Les formes spécifiques de l'internationalité du champ scientifique», Actes de la recherche en sciences sociales, $\mathrm{n}^{\text {os }} 141$ 142, mars 2002, p. 31-45, «L'université en mouvement», Égalité, (Revue de la Société acadienne d'analyse politique), no 50, automne 2004, p. 1328, et Éloge de l'homo techno-logicus, un essai publié en 2005.

François Girard travaille comme agent de recherche et de planification socioéconomique au ministère de l'Éducation, du Loisir et du Sport. Depuis plusieurs années, ce chercheur s'intéresse particulièrement à la mesure de la transition études et emplois. Dans ce cadre, il mène notamment des enquêtes, de type Relance, auprès des personnes diplômées des formations technique et universitaire. Il a dernièrement conduit le premier sondage auprès des employeurs de personnes récemment titulaires d'un diplôme universitaire (baccalauréat, maîtrise) qui, lors de l'enquête Relance, ont dit exercer un emploi en rapport avec leur formation.

Salim Laaroussi, diplômé de l'École polytechnique en génie électrique, a poursuivi ses études, au $2^{\mathrm{e}}$ cycle en robotique et a également agi à titre de président de l'Association des étudiants des cycles supérieurs de l'École 
polytechnique de Montréal (AECSP). Interpellé par les questions d'éducation et de recherche universitaire, il a poursuivi son engagement social et politique à la Fédération étudiante universitaire du Québec où il a occupé respectivement les postes de vice-président (2003-2004) et président (2004-2005) du CNCS. Au cours de ces deux mandats, le CNCS a notamment déposé et présenté le mémoire Le savoir, moteur de l'innovation. Pour un Québec qui se donne les moyens de son excellence en commission parlementaire, et a travaillé activement à la valorisation du statut d'étudiant-chercheur, tout en organisant le $1^{\text {er }}$ Salon national de la recherche universitaire (2005).

Wenceslas Mamboundou, Ph. D. en science politique de l'Université Laval, a été très engagé dans les associations étudiantes universitaires au Québec. Il a été président de l'Association des étudiantes et des étudiants de l'Université Laval inscrits aux études supérieures de 2001 à 2003. Il a été président du Conseil national des cycles supérieurs de la Fédération étudiante universitaire du Québec (CNCS-FEUQ) de 2003 à 2004 puis directeur à l'exécutif de la même organisation de 2004 à 2005.

Tout en se préoccupant des différentes problématiques que vivent les étudiants chercheurs au Québec et au Canada, il a rédigé de nombreux avis et mémoires sur le financement des études supérieures et de la recherche universitaire. Lors de la commission parlementaire sur le financement des universités au Québec, en 2004, il a co-rédigé et présenté le mémoire du CNCS intitulé: Le savoir, moteur de l'innovation. Pour un Québec qui se donne les moyens de son excellence.

Pierre Michel est un sociologue spécialisé en recherche quantitative. Il travaille depuis près de 25 ans au gouvernement du Québec. Il est l'un des concepteurs des enquêtes Relance conduites auprès des personnes diplômées de la formation professionnelle et de l'enseignement technique. et du Sondage auprès des employeurs qui est mené auprès des employeurs de recrues issues de la formation professionnelle, de l'enseignement technique et des universités québécoises. Depuis peu, il agit à titre de chef aux enquêtes et aux sondages au ministère de l'Éducation et est également responsable de la Relance à l'université. 
Daniel Parent, $\mathrm{Ph}$. D. en sciences économiques de l'Université de Montréal, est professeur agrégé et William Dawson Scholar au Département de science économique de l'Université McGill. Spécialisé en économie du travail et économétrie appliquée, il est chercheur au Centre interuniversitaire sur le risque, les politiques économiques et l'emploi (CIRPÉE) et Fellow au Centre interuniversitaire de recherche en analyse des organisations (CIRANO). Visiting Fellow à Princeton University en 1995, il a également été professeur adjoint au Département d'économique de l'Université de Sherbrooke.

Jean-Pierre Proulx, Ph. D. en théologie pastoral de l'Université de Montréal, est président du Conseil supérieur de l'éducation.

Journaliste au quotidien Le Devoir de 1968 à 1974 et de 1980 à 1991, spécialisé dans les questions religieuses et d'éducation, il a également été secrétaire du Comité sur la restructuration scolaire du Conseil scolaire de l'île de Montréal de 1974 à 1977, directeur du Bureau de l'admissibilité à l'enseignement en anglais du ministère de l'Éducation du Québec de 1977 à 1980, président du Groupe ministériel de travail sur la place de la religion à l'école de 1997 à 1999. Il est professeur à la Faculté des sciences de l'éducation de l'Université de Montréal depuis 1991 et directeur adjoint du Centre de formation initiale des maîtres de 1995-1999. Ses recherches et son enseignement portent sur les institutions scolaires québécoises, en particulier ce qui concerne leurs aménagements linguistiques et religieux, sur la démocratie scolaire locale et sur l'opinion publique en éducation.

Hélène P. Tremblay, $\mathrm{Ph}$. D. en sciences économiques de l'Université Laval et d'un post-doctorat de l'University of British Columbia, est présidente du Conseil de la science et de la technologie du Québec.

D'octobre 1999 à août 2000, elle a exercé les fonctions de directrice scientifique à l'Institut national de la recherche scientifique (INRS). Audelà des nombreuses fonctions qu'elle a assumées, madame Tremblay a également siégé sur le Conseil universitaire de l'Université Laval et a été membre de plusieurs comités, groupes de travail et conseils d'administration. Elle a été professeure d'économique à l'Université du Québec à Rimouski, vice-rectrice à l'enseignement et à la recherche et rectrice par intérim de la même université. Entre janvier 1997 et octobre 1999, elle a 
été sous-ministre adjointe Pêches et Aquiculture commerciales et sousministre adjointe Formation et Recherche au ministère de l'Agriculture, des Pêcheries et de l'Alimentation du Québec.

Possédant une bonne expérience sur les problématiques de la science et de l'innovation au Québec, madame Tremblay a participé a plusieurs colloques, conférences et tables rondes traitant des enjeux et des défis de l'innovation. 